\title{
Random Walks in Asymmetric Random Environments
}

\author{
J. Bricmont ${ }^{1}$ and A. Kupiainen ${ }^{2, \star}$ \\ 1 Physique Théorique, UCL, B-1348 Louvain-la-Neuve, Belgium \\ 2 Rutgers University, Department of Mathematics, New Brunswick, NJ 08903, USA \\ Received August 17, 1990; in revised form March 13, 1991
}

Dedicated to Joel Lebowitz on his 60th birthday

\begin{abstract}
We consider random walks on $\mathbf{Z}^{d}$ with transition rates $p(x, y)$ given by a random matrix. If $p$ is a small random perturbation of the simple random walk, we show that the walk remains diffusive for almost all environments $p$ if $d>2$. The result also holds for a continuous time Markov process with a random drift. The corresponding path space measures converge weakly, in the scaling limit, to the Wiener process, for almost every $p$.
\end{abstract}

\section{Introduction}

Random walks are probably the most extensively studied models of nonequilibrium behaviour. On a lattice $\mathbf{Z}^{d}$, a random walk is defined by a matrix $p(x, y), x, y \in \mathbf{Z}^{d}$ giving the probability of jumping from $x$ to $y$ at each time. The only constraints on $p$ are

$$
\begin{gathered}
p(x, y) \geqq 0, \\
\sum_{y} p(x, y)=1 \quad \forall x .
\end{gathered}
$$

Usually one considers walks on homogeneous environments, which means that

$$
p(x, y)=p(x-y) .
$$

For a random walk in a random environment (RWRE), $p$ is a random matrix. The randomness models the effect of impurities on a physical system, and one would like to study properties of the walk (e.g. its long time asymptotics) for almost every sample $p$.

Apart from its obvious interest in the study of diffusion in non-homogeneous media, RWRE may be considered as a simple model related to various other physical situations. These include Anderson's tight-binding model for disordered

* Supported by NSF-grant DMS-8903041 
electron systems, deterministic motion among random scatterers (the Lorentz gas), or the time evolution of disordered systems.

In this paper we consider a RWRE, where $p$ is a small short range random perturbation of a homogeneous walk. We can deal with rather generic such perturbations, but the most interesting is the one where the environment is asymmetric: $p(x, y)$ and $p(y, x)$ are independent. For such walks we show that normal diffusive behaviour takes place if $d>2$ : the diffusion constant is non-zero and independent of $p$ for almost all $p$. Also, the (long time) scaling limit of the walk is the Wiener process.

The continuous time analog of random walk is Brownian motion. The analog of RWRE is the Markov process (on the state space $\mathbf{Z}^{d}$ ), with generator

$$
\Delta+\vec{b} \cdot \vec{\nabla}
$$

where $\vec{b}$ is random. Thus, the transition probability $P(x, t)$ in time $t$ from origin to $x$ satisfies the Fokker-Planck equation

$$
\partial_{t} P=\Delta P-\vec{\nabla} \cdot(\vec{b} P)
$$

Our results extend to such Markov processes for a distribution of $\vec{b}$ whose covariance equals

$$
E\left(b_{\alpha}(x) b_{\beta}(y)\right)=\varepsilon^{2} \delta_{\alpha \beta} \delta_{x y}
$$

with $\varepsilon$ small, and $d>2$.

One of the first results on asymmetric RWRE is due to Sinai [1]. He showed that, in one dimension, the effect of the asymmetry in the environment is drastic: the mean square displacement is typically of the order $(\log t)^{4}$, instead of $t$ in the homogeneous environment.

This result prompted investigations and some controversy about the behaviour in higher dimensions. Some numerical work [2] indicated logarithmic behaviour in $d=2$, for strong disorder. Subsequently, perturbative renormalization group computations were performed [3-5] which gave support to diffusive behaviour in $d \geqq 2$. The argument showed that the zero disorder (homogeneous) fixed point is stable: upon scaling space and time, the effective disorder renormalizes to zero. On dimensional grounds this is easy to understand for $d>2$. If we define $P^{\prime}(x, t)$ $=L^{d} P\left(L x, L^{2} t\right)$, where $P$ solves (5), then $P^{\prime}$ solves (5) with a new noise $\overrightarrow{b^{\prime}}=L^{d} \vec{b}\left(L x^{\prime}\right)$. If we replace the state space by $\mathbf{R}^{d}$ and if $\vec{b}$ is white noise, then

$$
E\left(b_{\alpha}^{\prime}(x) b_{\beta}^{\prime}(y)\right)=\varepsilon^{2} L^{2-d} \delta_{\alpha \beta} \delta(x-y)
$$

Of course, to make sense of this argument one has to regularize the delta function and replace scaling by a renormalization group (RG) analysis. In $d=2$, a second order computation in the disorder reveals the irrelevancy of the disorder [3]. Of course the perturbative $\mathrm{RG}$ can be argued to be reliable at most for small disorder. However, in [3], it was argued that the large disorder fixed point is unstable under the RG, and, thus, this gives plausibility to the claim that the walk is diffusive for all (local) disorder.

This claim was subsequently challenged $[6,8]$ by explicit counterexamples. In [6], environments with long-range correlations were constructed which have logarithmic behaviour like the one found by Sinai in $d=1$. These models do not fit into the framework discussed above due to the non-localities. However, they can be understood in the RG framework [7] at least heuristically. 
An important problem in the asymmetric walk is the presence of traps, i.e. regions where the walk can remain trapped for long times. Such traps will occur in all distance scales and, if too abundant, they may produce sub-diffusive behaviour. These traps occur in local models and were used in the counterexample of [8]. However, as we explain below (see also [7]), such counterexamples again fit very well in the RG picture.

The trapping properties of the asymmetric environments are absent in models of RWRE in symmetric environments, where e.g. $p(x, y)=p(y, x)$. For continuous time, this means that the random force $\vec{b}$ is the gradient of a stationary random potential $\vec{b}=\vec{\nabla} v$. If $v$ is white noise, then, in the formal scaling argument (7), the power of $L$ is replaced by $-d$, i.e. the noise is irrelevant in all dimensions. This is indeed the case: in [9 and 10] it was shown that the diffusion is normal in all dimensions and without our restriction on $\varepsilon$ being small. These results were subsequently extended to various lattice models [11-14].

Our proof is outlined in detail in Sect. 3. It is based on a RG analysis, somewhat similar to the one in [15]. The RG transformation consists of decimation in time, combined with the scaling of space and time. This transformation maps a RWRE to another RWRE, with local transition probabilities, but weaker randomness, as in (7). Iteration of the $R G$ drives the system to a fixed point, given by the Wiener process, which describes the long time asymptotics of the original RWRE.

The method should extend to $d=2$, where a more detailed study of the RG in the small disorder region is required. The proof given below works, formally, in $d=2+\varepsilon$ for all $\varepsilon>0$. We hope to be able to use the method to study the emergence of stochastic (diffusive) behaviour in deterministic models such as the Lorenz gas and lattice versions thereof.

In Sect. 2, we state our results. The ideas of the proof are explained in detail in Sect. 3, where the outline of the rest of the paper is given.

\section{The Model and the Results}

A random walk on $\mathbf{Z}^{d}$ is described by the transition probabilities $p(x, y)$ from $x \in \mathbf{Z}^{d}$ to $y \in \mathbf{Z}^{d}$ :

$$
p: \mathbf{Z}^{d} \times \mathbf{Z}^{d} \rightarrow[0,1]
$$

satisfying

$$
\sum_{y \in \mathbf{Z}^{d}} p(x, y)=1
$$

$p$ allows us to define measures $\mu_{T}, T \in \mathbf{N}$ on the space $\Omega_{T}$ of walks $\omega:\{0,1, \ldots, T\} \rightarrow \mathbf{Z}^{d}$ starting from $\omega(0)=0$ :

$$
\mu_{T}(\omega)=\prod_{i=1}^{T} p(\omega(i-1), \omega(i))
$$

We will study in this paper the large $T$ properties of such measures. It will be convenient to realize them as measures $v_{T}$ on $C([0,1])$, the space of continuous paths $\omega:[0,1] \rightarrow \mathbf{R}^{d}$, by rescaling the time in a standard way. Thus, given an $\omega \in \Omega_{T}$, we obtain a piecewise linear path

$$
\omega(t)=T^{-1 / 2}(\omega(i-1)+(T t-i+1)(\omega(i)-\omega(i-1))),
$$


where $i-1=[T t]$ and [] denotes the integral part. $v_{T}$ is the measure induced by (4) on $C([0,1])$, and we will study the limit

$$
\lim _{T \rightarrow \infty} v_{T}
$$

also called the scaling limit, and its properties. For convenience, we will consider below times given by $T=L^{2 n}$ for $n \in \mathbf{N}$ and $L$ a fixed integer chosen later. We will denote $v_{L^{2 n}}$ by $v_{n}$ for short.

A random walk in a random environment is a random walk for which the probabilities $p(x, y)$ satisfying (1) and (2) are random variables with a given probability distribution. We then investigate the existence of the limit (5) almost surely with respect to this distribution.

Let us now specify precisely the properties of $p$ which we impose. For simplicity, we consider nearest neighbor walks (see, however, Remark 1 after Theorem 2):

$$
p(x, y)= \begin{cases}\frac{1}{2 d}+b(x, y) & |x-y|=1 \\ 0 & |x-y| \neq 1 .\end{cases}
$$

For $b=0,(6)$ defines the simple random walk. From (2) we see that

$$
\sum_{y} b(x, y)=0 \text {. }
$$

The $\mathbf{b}=\{b(x, y)\}_{x, y \in \mathbf{Z}^{d}}$ is a family of random variables whose distribution $\mathscr{P}$ we now describe.

(P1) Independence. We take $b(x, y)$ and $b\left(x^{\prime}, y^{\prime}\right)$ to be independent if $x \neq x^{\prime}$ : order the unit vectors in $\mathbf{Z}^{d}$ in some arbitrary way: $\left(e_{1}, e_{2}, \ldots, e_{2 d}\right)$. Put

$$
b(x)=\left(b\left(x, x+e_{1}\right), \ldots, b\left(x, x+e_{2 d}\right)\right) \in \mathbf{R}^{2 d} .
$$

We take $\{b(x)\}_{x \in \mathbf{Z}^{d}}$ to be independent, identically distributed random variables with mean zero

$$
E b(x)=0
$$

satisfying (7) and (1) for $p$ of (6). Note, in particular, that $b(x, y)$ and $b(y, x)$ are independent: the environment is asymmetric

(P2) Isotropy. Let $R \in O(d)$ be a rotation of $\mathbf{R}^{d}$ fixing the lattice $\mathbf{Z}^{d}$. This induces a permutation $\pi \in S_{2 d}$ of the $e_{i}$ 's, and thus a permutation of the coordinates of $b(x)$, $\pi^{*} b(x)$. Then, we require that

$$
b(x) \text { and } \pi^{*} b(x)
$$

are identically distributed (note that this and (7) imply (9)).

We next require that $b$ in (6) is a "small" perturbation in the following sense.

(P3) The generating function of $b$ satisfies

$$
E e^{t b(x, y)} \leqq e^{t^{2} \varepsilon^{2}}
$$

Finally, we impose a condition on the probability that the $p(x, y)^{\prime} s$ are near zero:

(P4)

$$
\operatorname{Prob}\left(p(x, y) \leqq \frac{1}{2 d} e^{-N}\right) \leqq e^{-\Gamma N}, \quad N \in \mathbf{N}
$$


This is designed to avoid the walk getting "trapped" in some region of $\mathbf{Z}^{d}$, see the discussion in Sect. 3; $\varepsilon$ in (11) will be taken small and $\Gamma$ in (12) large.

We now state the main result concerning the limit (4). Let us denote by

$$
T_{D}^{*}(x)=\left(2 \pi \frac{D}{d}\right)^{-d / 2} \exp \left[-\frac{d x^{2}}{2 D}\right]
$$

the transition probability density for the Wiener measure $v^{D}$ with diffusion constant $D$. The scaling limit of our walk is given by $v^{D}$ for almost all environments. The convergence takes place in at least in two senses: first of all, as Theorem 1 shows, suitable correlation functions converge, and this implies convergence of the diffusion constant and of the finite dimensional distributions (take $f(x)=e^{i k x}$ below, and use Theorem 7.6 in [16]). We consider the following class of smooth functions:

$$
\mathscr{F}=\left\{f \in C^{\infty}\left(\mathbf{R}^{d}\right)|| f(x)|+| \nabla f(x) \mid \leqq C e^{|x|^{1 / 2}}\right\}
$$

Then,

Theorem 1. Let $\mathscr{P}$ satisfy (P1)-(P4) and $d>2$. Then there is an $\varepsilon_{0}>0$ and $\Gamma_{0}$ such that for $\varepsilon<\varepsilon_{0}, \Gamma>\Gamma_{0}$ there exists a $D>0$ such that the limit

$$
\lim _{n \rightarrow \infty} \int d v_{n}(\omega) \prod_{i} f_{i}\left(\omega\left(t_{i}\right)\right)
$$

exists and is given by

$$
\int d v^{D}(\omega) \prod_{i} f_{i}\left(\omega\left(t_{i}\right)\right)
$$

for any $f_{1} \ldots f_{k} \in \mathscr{F}$, and $t_{1} \ldots t_{k} \in[0,1] \mathscr{P}$-almost surely. Moreover, $D$ satisfies

$$
|D-1| \leqq c \varepsilon^{2} \text {. }
$$

Let us define the diffusion constant in time $T=L^{2 n}$ by

$$
D_{n}(p)=\frac{1}{T} \sum_{\omega} \mu_{T}(\omega) \omega(T)^{2}
$$

and

$$
D(p)=\lim _{n \rightarrow \infty} D_{n}(p)
$$

Then,

Corollary. The limit (17) exists $\mathscr{P}$-a.s. and equals

$$
D(p)=D \text {, }
$$

where $D$ is given in Theorem 1.

Indeed, (16) equals

$$
D_{T}(p)=\int d v_{T}(\omega) \omega(1)^{2}
$$

whereas

$$
D=\int d v^{D}(\omega) \omega(1)^{2}
$$


Actually, the convergence to the Wiener measure also holds for all bounded continuous functions on the path space, not only factorisable ones, as in Theorem 1:

Theorem 2. Under the assumptions of Theorem 1,

$$
v_{T} \rightarrow v^{D}
$$

weakly, $\mathscr{P}$-almost surely. $D$ is the same as in Theorem 1.

Remark 1. Theorems 1 and 2 hold for a much more general class of RWRE's than described above. While the general case is described by our inductive assumptions, our results extend, for example, to the following class of models: we assume

$$
p(x, y)=T(|x-y|)+b(x, y)
$$

with

$$
\begin{gathered}
0 \leqq T(x) \leqq C e^{-|x|}, \quad|b(x, y)| \leqq C e^{-|x-y|}, \\
\sum_{x} T(x)=1
\end{gathered}
$$

and

$$
E b(x, y)=0=\sum_{y} b(x, y) .
$$

The random fields $b(x)=\{b(x, y)\}_{y \in \mathbf{Z}^{d}}$ satisfy

(a) $b(x), b\left(x^{\prime}\right)$ are independent, identically distributed.

(b) $G(f) \equiv E \exp \left(\sum_{y} b(0, y) f(y)\right) \leqq \exp \varepsilon^{2} \sum_{y} e^{-|y|} f(y)^{2}$.

(c) (Isotropy) $G(f)=G\left(R^{*} f\right)$, where $R \in O(d)$ fixes $Z^{d}$.

(d) $\operatorname{Prob}\left(p(0, y)>1-e^{-N}\right)<e^{-N \Gamma} ; N \geqq 1$.

Remark 2. The analysis covers also a continuous time version of RWRE. Let $P^{t}(x, y, \beta)$ be the solution of

$$
\frac{d P^{t}}{d t}=(\Delta-\nabla \cdot \beta) P^{t}, \quad P^{0}(x, y, \beta)=\delta_{x y}
$$

with $\Delta$ the Laplacian on $\mathbf{Z}^{d}, \nabla$ the finite difference operator and let $\beta_{i}(x), i=1, \ldots, d$, $x \in \mathbf{Z}^{d}$ be independent identically distributed random variables with mean zero and

$$
\left|\beta_{i}(x)\right|<\varepsilon .
$$

This problem essentially reduces to the previous $t \in \mathbf{N}$ case by putting

$$
p(x, y)=e^{\Delta-\nabla \cdot \beta}(x, y) .
$$

Clearly $\sum_{y} p(x, y)=1$ and $p(x, y) \geqq 0$. Furthermore, $p$ is analytic in $\left\{\beta_{i}(x)\right\}_{i, x}$ in the polydisc $(21)$ (as a bounded operator on $l^{2}\left(\mathbf{Z}^{d}\right)$ ) with

$$
\left|\prod_{\alpha=1}^{N} \frac{\partial p}{\partial \beta_{i_{\alpha}}\left(x_{\alpha}\right)}\right|_{\beta=0} \mid \leqq c^{N} \exp \left[-d\left(\cup x_{\alpha} \cup x \cup y\right)\right] \prod_{\beta} n_{\beta} !,
$$

where $n_{\beta}$ are the multiplicities of $\beta_{i}(x)^{\prime} s$, and $d(X)$, for $X \subset \mathbf{Z}^{d}$, is the length of the shortest connected graph on $X ;(23)$ may be obtained via a repeated application of 
Duhamel's formula and of

Therefore

$$
e^{t \Delta}(x, y) \leqq c e^{-|x-y| t^{-1 / 2}} .
$$

and

$$
p(x, y)=E p(x, y)+\sum_{Y \subset \mathbf{Z}^{d}} b_{Y}(x, y)
$$

$$
\left|b_{Y}(x, y)\right| \leqq(c \varepsilon)^{|Y|} \exp [-d(x \cup y \cup Y)] .
$$

These $p(x, y)^{\prime} s$ fit into our inductive assumptions. Here $b_{Y}$ collects terms in the Taylor series of $p$ with $\beta_{i}(x), x \in Y$. Thus the $b_{Y_{i}}$ 's are independent for disjoint $Y_{i}$ 's.

\section{The Renormalization Group - Outline of the Proof}

\section{The RG Transformation}

The RG will allow us to replace the analysis of long time properties of the walk by the study of a map, the RG map, relating transition probability densities of successive scales.

Given a matrix $p(x, y)$ satisfying (2.1) and (2.2) and thus defining a random walk, choose an integer $L>1$ and set

$$
p_{1}(x, y)=L^{d} \sum_{\omega} \prod_{i=1}^{L^{2}} p(\omega(i-1), \omega(i)) \equiv L^{d} p^{L^{2}}(L x, L y)
$$

for $x, y \in\left(L^{-1} \mathbf{Z}\right)^{d}$. In the sum, $\omega(0)=L x, \omega(T)=L y$ and we write $|\omega|=L^{2}$. Let $P^{T}(x, y, p)$ be the probability, in the random walk defined by $p$, to go from $x$ to $y$ in time T. Explicitly

$$
P^{T}(x, y, p)=\sum_{\omega} \prod_{i=1}^{T} p(\omega(i-1), \omega(i))
$$

with $\omega(0)=x, \omega(T)=y$.

With definition (1) and $T=L^{2 n}$, we have

$$
\begin{aligned}
P^{T}(x, y, p) & =L^{-d} P^{T_{1}}\left(L^{-1} x, L^{-1} y, p_{1}\right) \\
& =L^{-d} \sum_{\omega: \omega(i) \in\left(L^{-1} \mathbf{Z}\right)^{d}} L^{-d\left(T_{1}-1\right)} \prod_{i=1}^{T_{1}} p_{1}(\omega(i-1), \omega(i)),
\end{aligned}
$$

where $\omega(0)=L^{-1} x, \omega\left(T_{1}\right)=L^{-1} y, T_{1}=L^{-2} T$. The powers of $L$ in (1) are of course chosen because we expect the long time limit to be diffusive. The ones in (3) become very natural, provided we note that, since $\omega$ now are walks in $\left(L^{-1} \mathbf{Z}\right)^{d}$, due to the scaling involved in $p_{1}$, it is natural to replace $\sum_{\omega}$ by an "integral." Therefore, we will shift to the following notation:

$$
\int d \omega_{x, y}^{t} \equiv \int_{L^{-1} \mathbf{Z}^{d}} \prod_{i=0}^{t} d \omega(i) \delta(\omega(0)-x) \delta(\omega(t)-y),
$$

where

$$
\int_{\left(L^{-1} \mathbf{Z}\right)^{d}} d \omega(i)=L^{-d} \sum_{\omega(i) \in\left(\mathbf{L}^{-1} \mathbf{Z}\right)^{d}}
$$


and

$$
\delta(\omega(0)-x)=L^{d} \delta_{\omega(0), x} .
$$

Equations (4-6) will shortly be used with $L$ replaced by $L^{n}, n \in \mathbf{N}$. Thus (3) reads

$$
\begin{gathered}
P^{T_{1}}\left(x_{1}, y_{1}, p_{1}\right)=\int d \omega_{x_{1} y_{1}}^{T_{1}} p_{1}(\omega), \\
p_{1}(\omega)=\prod_{i=1}^{|\omega|} p_{1}(\omega(i-1), \omega(i)) .
\end{gathered}
$$

Equations (2) and (3) are of course nothing but a convenient rewriting of $P^{T}$. It is obvious that we may iterate this operation, to obtain

$$
P^{T}(x, y, p)=L^{-n d} P^{L^{-2 n} T}\left(L^{-n} x, L^{-n} y, p_{n}\right),
$$

where the right-hand side refers to walks on $L^{-n} \mathbf{Z}^{d}$ with transition probabilities $p_{n}: L^{-n} \mathbf{Z}^{d} \times L^{-n} \mathbf{Z}^{d} \rightarrow[0,1]$. Indeed, we have the recursion

$$
p_{n+1}(x, y)=L^{d} \int d \omega_{L x L y}^{L^{2}} p_{n}(\omega),
$$

where the right-hand side involves (4-6) with $L \rightarrow L^{n}$. Clearly, the property

$$
\int d y p_{n}(x, y)=1
$$

is preserved under the map $(10) ;(10)$ is the Renormalization Group map. It maps an "environment" $p_{n}$ to another $p_{n+1}$. Thus $p_{n}, n \geqq 1$, are random variables, being functions of $p$. The meaning of $(10)$ is that the rescaled long-time transition probabilities for our RWRE are given as the transition probabilities in the rescaled time of a RWRE with renormalized p's. Given (11), it may be useful to think of $p_{n}(x, y)$ as a transition probability density, in $R^{d}$, which is constant on cubes of side $L^{-n d}$. To solve for the scaling limit we need to solve for the $p_{n}^{\prime} s$.

Consider for example the diffusion constant (2.16). We get from (2.16) and (9)

$$
D_{n}(p)=D_{0}\left(p_{n}\right)=\int d y p_{n}(0, y) y^{2} .
$$

Thus the long time behavior is reduced to a time 1 problem for $p_{n}$, as $n \rightarrow \infty$.

The next step consists in dividing $p_{n}$ into a "deterministic" and a "random" part:

$$
p_{n}(x, y)=T_{n}(x-y)+b_{n}(x, y)
$$

where

$$
\begin{gathered}
T_{n}(x-y)=E p_{n}(x, y), \\
b_{n}(x, y)=p_{n}(x, y)-E p_{n}(x, y),
\end{gathered}
$$

and we used the translation invariance of the distribution of $b$. Evidently, by (11),

$$
\begin{gathered}
\int d y T_{n}(y)=1, \\
\int d y b_{n}(x, y)=0=E b_{n}(x, y) .
\end{gathered}
$$

The bulk of this paper consists in showing that $b_{n}$ tends to zero as $n \rightarrow \infty$, whereas $T_{n}$ tends to (2.13). Let us consider $T_{n}$ first. The iteration (10) is very easy to solve if $b=0$ : we have a convolution and denoting by $\hat{T}$ the Fourier transform of $T$, we have

$$
\widehat{T}_{n+1}(k)=\widehat{T}_{n}\left(\frac{k}{L}\right)^{L^{2}}
$$


i.e.

$$
\widehat{T}_{n}(k)=\hat{T}\left(\frac{k}{L^{n}}\right)^{L^{2 n}}
$$

where, from (2.6)

$$
\hat{T}(k)=\frac{1}{d} \sum_{\alpha=1}^{d} \cos k_{\alpha} .
$$

Hence, as $n \rightarrow \infty$,

$$
\widehat{T}_{n}(k) \rightarrow \exp \left[-\frac{k^{2}}{2 d}\right] \equiv \hat{T}_{1}^{*}(k)
$$

where

$$
T_{D}^{*}(x)=\left(2 \pi \frac{D}{d}\right)^{-d / 2} e^{-d x^{2} / 2 D} .
$$

Of course $b$ is not zero and, at each scale, $b_{n}$ will modify the diffusion constant. Since $b_{n}$ goes to zero, we shall obtain a sequence of approximations $D_{n}$, given by $E\left(D_{1}\left(p_{n}\right)\right)$, see $(12)$, to the true diffusion constant $D$.

\section{The Flow of the Disorder}

Now, let us see in what sense $b_{n}$ goes to zero. The recursion (10), written for $b$, reads, using (15)

$$
b_{n+1}(x, y)=\left(R b_{n}\right)(x, y) \equiv L^{d} \int d \omega_{L x L y}^{L^{2}}\left[\left(T_{n}+b_{n}\right)(\omega)-E\left(T_{n}+b_{n}\right)(\omega)\right] .
$$

Equation (23) defines the renormalization group map $R$ for the stochastic part $b$ of $p$. The main difference between $b_{n}$ and $b$ of $(2.6)$ is that $b_{n}(x, y), b_{n}\left(x^{\prime}, y^{\prime}\right)$ are no longer independent. Indeed, we have

$$
b_{n}(x, y)=L^{n d} \int d \omega_{L^{n} x L^{n} y}^{L^{2 n}}\{(T+b)(\omega)-E[(T+b)(\omega)]\},
$$

which shows that $b_{n}(x, y)$ depends on $b(\tilde{x}, \tilde{y})$ for

$$
\left|L^{n} x-\tilde{x}\right|+\left|L^{n} y-\tilde{x}\right| \leqq L^{2 n}
$$

Also, $p_{n}$ are not strictly local: $p_{n}(x, y)=0$ only if

$$
|x-y|>L^{n} \text {. }
$$

Of course, we expect $b_{n}(x, y)$ and $b_{n}\left(x^{\prime}, y^{\prime}\right)$ to be only weakly dependent if $\left|x-x^{\prime}\right|$ is large, and be exponentially small if $|x-y|$ is large. The third difference is that $b_{n}$ lives on $L^{-n} \mathbf{Z}^{d}$, i.e. we have a proliferation of variables: obviously $b_{n}(x, y)$ and $b_{n}\left(x^{\prime}, y^{\prime}\right)$ should be "almost the same" if $\left|x-x^{\prime}\right|$ and $\left|y-y^{\prime}\right|$ are very small; in particular, they will not be independent but, rather, strongly correlated.

These problems are solved by localizing $b_{n}(x, y)$ in terms of which $b(\tilde{x}, \tilde{y})^{\prime} s$ it depends on. We write

$$
b_{n}(x, y)=\sum_{Y \subset \mathbf{Z}^{d}} b_{n Y}(x, y)
$$


where $b_{n Y}(x, y)$ are random variables that are functions of $b(\tilde{x}, \tilde{y})$ for $\tilde{x} \in L^{n} \mathbf{Y}$ (more precisely, in a neighborhood of this set, see below). Y denotes the union of unit cubes in the lattice of spacing $L^{-n}$ centered at $y \in Y$. An expression like (27) would result from (24) upon expanding the $b$ 's:

$$
\begin{aligned}
(T+b)(\omega) & =\prod_{i}(T+b)\left(\omega_{i-1}, \omega_{i}\right) \\
& =\sum_{\left(n_{i}\right)} \sum_{\left(x_{i}\right)\left(y_{i}\right)} T^{n_{1}}\left(L^{n} x-x_{1}\right) b\left(x_{1}, y_{1}\right) T^{n_{2}}\left(y_{1}-x_{2}\right) b\left(x_{2}, y_{2}\right) \ldots b\left(x_{k}, L^{n} y\right)
\end{aligned}
$$

and letting $b_{n Y}(x, y)$ be the sum of the terms for which $\mathbf{Y}$ is the smallest set of cubes such that $\cup x_{i} \subset L^{n} \mathbf{Y}$. We cannot however proceed to straightforwardly. There are two reasons for this.

First, the expansion (28) is not a good thing to do, if some $b(\tilde{x}, \tilde{y})$ is not small, i.e. in particular if $T+b$ is near 0 or 1 . This is the problem of traps: configurations of $b^{\prime} s$ that will cause the walk to be trapped in some region in $\mathbf{Z}^{d}$.

Second, even if all the $b^{\prime}$ s are typical, i.e. of order $\varepsilon$, the expansion (28) turns out to be uncontrollable, due to multiple scales. This means that even small initial $b(x, y)^{\prime} s$ can give rise to a $b_{n}$ at some later scale, which is not a small perturbation of $T_{n}$ : traps can occur in longer scales as a collective conspiracy of small $b(x, y)^{\prime} s$. This mechanism was exploited in [8] in order to provide examples of RWRE with subdiffusive behaviour.

We now describe these two problems in turn and the ways we deal with them.

First, consider the RG map (23), for $n=0$ (the first step) and all $b(\tilde{x}, \tilde{y})$ entering in $b_{1}(x, y)$ small:

$$
|b(\tilde{x}, \tilde{y})|<\delta,
$$

where $\delta \ll 1$. Provided $\delta \gg \varepsilon,(29)$ is probable. Then, to first order in $b$,

$$
b_{1}\left(x^{\prime}, y^{\prime}\right)=L^{d} \sum_{x, y} \sum_{t=0}^{L} T^{t}\left(L x^{\prime}-x\right) T^{L^{2}-t}\left(L y^{\prime}-y\right) b(x, y) .
$$

Let us localize (30) in $b$ as indicated above. We define, for $u \in \mathbf{Z}^{d}$,

$$
b_{1 u}\left(x^{\prime}, y^{\prime}\right)=L^{d} \sum_{x \in L \mathbf{u}} \sum_{y} \sum_{t=0}^{L^{2}} T^{t}\left(L x^{\prime}-x\right) T^{L^{2}-t}\left(L y^{\prime}-y\right) b(x, y),
$$

where $L \mathbf{u}$ is the $L^{d}$-cube centered at $L u$ (note that $b_{1 u}$ is taken to be localized in $L \mathbf{u}$ due to the scaling by $L$ ). Clearly, $b_{1 u_{1}}$ and $b_{2 u_{2}}$ are independent if $u_{1} \neq u_{2}$.

Let us inquire the variance of (31). We will study later in great detail the linearized RG map (30), so let us now just get a qualitative picture of it. The free walk part $T^{t}$ satisfies

$$
T^{t}(x) \leqq \frac{c}{t^{d / 2}} \exp \left[-2|x| / t^{1 / 2}\right] ; \quad t>0
$$

(the 2 is arbitrary: the true behavior is rather as $\exp \left[-c x^{2} / t\right]$ but we won't need such accuracy here). Consider for example the case $x^{\prime}=y^{\prime}=u=0$. Then, we walk freely from 0 to $x$ in time $t$ and from $x$ back to 0 in time $L^{2}-t$. The walk, in time $\leqq L^{2}$ predominantly stays near the $L^{d}$-cube $L \mathbf{0}$, hits a particular $x$ with probability $L^{-d}$ at a given time $t$ and 0 again with probability $L^{-d}$. Altogether, summing over $L^{2}$ 
times, we have

$$
(31) \sim C L^{2-d} \sum_{x \in L \mathbf{0}} \sum_{|y-x|=1} K(x, y) b(x, y)
$$

with

$$
|K(x, y)|<1 .
$$

Being the sum of $L^{d}$ independent random variables, (33) then seems to have variance

$$
E b_{10}(0,0)^{2} \sim C L^{4-d} \varepsilon^{2} .
$$

This calculation seems to indicate that the randomness becomes more relevant in longer scales if $d<4$. However, we have ignored a very important property of $b$, namely (2.7): $\sum_{y} b(x, y)=0$. We may take advantage of this in (31) by replacing $T^{L^{2}-t}\left(L y^{\prime}-y\right)$ there by

$$
T^{L^{2}-t}\left(L y^{\prime}-y\right)-T^{L^{2}-t}\left(L y^{\prime}-x\right)
$$

for the terms with $L^{2}-t \neq 0$. But, since $|y-x|=1,(35)$ equals $\nabla_{x} T^{L^{2}-t}\left(L y^{\prime}-x\right)$ and it is not hard to bound it by (see (32))

$$
\left|\nabla_{x} T^{L^{2}-t}\left(L y^{\prime}-x\right)\right| \leqq C\left(L^{2}-t\right)^{-\frac{d+1}{2}} e^{-2\left|L y^{\prime}-x\right| /\left(L^{2}-t\right)^{1 / 2}} .
$$

Hence, for most $t$ ((i.e. $L^{2}-t>a L^{2}$, for some constant $a$ ), we gain a factor of $L^{-1}$ in (33) and therefore an $L^{-2}$ in (34): The contribution to $E b_{10}(0,0)^{2}$ from the terms in (31) with $t<(1-a) L^{2}$ is bounded by

$$
C(a) L^{2-d} \varepsilon^{2}
$$

i.e. the randomness is irrelevant (in the RG-sense) in $d>2$. We would then expect the variance of $b_{n}$ to run down as $\left(C L^{2-d}\right)^{n}$ (with $C$ an $L$-independent constant, $C L^{2-d}$ can be taken less than one for a suitable $L$ ). It is also clear from (32) and (31) that, for general $u, x^{\prime}, y^{\prime}$, the bound (37) will be multiplied by a factor

$$
\exp \left[-\left|x^{\prime}-u\right|-\left|y^{\prime}-u\right|\right]
$$

i.e. $b_{1}$ remains local with exponential tails.

There is, however, a slight catch in the above analysis that will cause us a lot of technical headache: namely the subtraction (35) will not help us for $t$ near $L^{2}$; we have stressed this by the $a$-dependence in (37). As an example of the problem, consider the $t=L^{2}$ term in (31), again for $x^{\prime}=y^{\prime}=u=0$; this is just

$$
\beta \equiv L^{d} \sum_{|x|=1} T^{L^{2}}(x) b(x, 0)
$$

whose variance is

$$
E \beta^{2}=2 d\left(L^{d} T^{L^{2}}(x)\right)^{2} E b(x, 0)^{2}
$$

for any $x$, with $|x|=1$. From (32) we see that no power of $L$ is gained in (40), in any dimension! $\left[\right.$ Actually, as $L \rightarrow \infty, L^{d} T^{L^{2}}(x) \rightarrow \int_{\mathbf{R}} e^{-k^{2} / 2 d} \frac{d^{d} k}{(2 \pi)^{d}}=\left(\frac{d}{2 \pi}\right)^{d / 2}$, so the prefactor in (40) is greater than one in high dimensions! ] Hence, pointwise, in $x, y$, we 
cannot expect

$$
E b_{n u}(x, y)^{2}
$$

to become small. Actually, we will later see that (41) stays bounded, as $n \rightarrow \infty$, but only by $\mathcal{O}\left(\varepsilon^{2}\right)$, if $y \in u$ (the precise bound is slightly weaker, see (61) below). The reader should realize that we have here a real problem for the whole RG scheme. If (41) does not contract as $n \rightarrow \infty$, then, $T_{n}$, given by (14), does not contract either: it is recursively given by

$$
\begin{aligned}
T_{n+1}\left(x^{\prime}-y^{\prime}\right) & =E L^{d} \int d \omega_{L x^{\prime} L y^{\prime}}^{L^{2}}\left(T_{n}+b_{n}\right)(\omega) \\
& =L^{d} T_{n}^{L^{2}}\left(L x^{\prime}-L y^{\prime}\right)+E \mathcal{O}\left(b_{n}^{2}\right),
\end{aligned}
$$

where the first term tends to stabilize to the fixed point (22), whereas the effect of the second term would be to change $D$ by $\mathcal{O}\left(\varepsilon^{2}\right)$ at each iteration step. Hence we could not control the diffusion constant, in particular, keep it away from zero.

The way out of this problem is to realize that the random variable (39), if we reinsert the $y^{\prime}\left(=0\right.$ in (39)), satisfies $\sum_{y^{\prime}} \beta\left(y^{\prime}\right)=0$. Therefore, although $b_{n u}(x, y)$ will not be smaller than $\mathcal{O}\left(\varepsilon^{2}\right)$ with high probability pointwise, it actually will be highly oscillating, and, when smeared with smooth test functions (what this means on a lattice, see below), actually has a variance running down as $L^{-\kappa n} \varepsilon^{2}$. We will see that, as a consequence, $T_{n}$ will be smooth enough, such that when $b_{n}$ is smeared with $T_{n}$, the $E \mathcal{O}\left(b_{n}^{2}\right)$ in (42) actually will be $\mathcal{O}\left(L^{-\kappa n} \varepsilon^{2}\right)$. Thus, once smeared with "nice enough" functions, $b_{n}$ has small variance, which inductively will guarantee that $T_{n}$ stays "nice enough."

To deal with such oscillations, Fourier transform is a natural tool. Recall that

$$
b_{n}: L^{-n} \mathbf{Z}^{d} \times L^{-n} \mathbf{Z}^{d} \rightarrow \mathbf{R}
$$

and we expect $b_{n}(x, y)$ to have exponential falloff as $|x-y|$ becomes large, whereas it will not be very smooth in $y$. Hence, it is natural to introduce a "mixed" representation for such functions: retaining the lattice description for scales $\geqq 1$ and using Fourier transform for smaller distance scales. Thus, denote by $\xi$ a pair

$$
\xi=(u, k), \quad u \in \mathbf{Z}^{d}, k \in 2 \pi \mathbf{Z}^{d},\left|k_{\mu}\right|<L^{n} \pi .
$$

Then, given

$$
f: L^{-n} \mathbf{Z}^{d} \rightarrow \mathbf{R}
$$

we put

$$
f(\xi)=\int_{\mathbf{u}} d x e^{i k(x-u)} f(x)
$$

where $\mathbf{u}$, for $u \in \mathbf{Z}^{d}$, denotes the unit cube in $L^{-n} \mathbf{Z}^{d}$ centered at $u$.

Now, the random variables $b_{n}$ will be split into

$$
b_{n}=s_{n}+r_{n},
$$

where $s_{n}$ will be the "small-field variables," ones describing randomness on scale $n$ outside the "trapping region" (see below), whereas $r_{n}$ describes the effect of the traps. $r_{n}$ will be "large" (albeit they are very improbable, see below) whereas $s_{n}$ will be "small" in the following sense. 


\section{The Small Fields}

We shall have

$$
S_{n}: L^{-n} \mathbf{Z}^{d} \times L^{-n} \mathbf{Z}^{d} \rightarrow \mathbf{R}
$$

with

$$
S_{n}(x, y)=\sum_{Y \subset \mathbf{Z}^{d}} S_{n Y}(x, y) .
$$

The $s_{n Y}$ satisfy the following properties.

(A) Independence. $S_{n Y}(x, y)$ is a function of $b(\tilde{x}, \tilde{y})$ for $L^{-n} \tilde{x}$ in a neighbourhood of $\mathbf{Y}$, where $\mathbf{Y}$ is defined, like $\mathbf{u}$ in (45), as the union of unit cubes centered at points of $Y$. In particular, $s_{n Y_{1}}\left(x_{1}, y_{1}\right), s_{n Y_{2}}\left(x_{2}, y_{2}\right)$ are independent if

$$
\operatorname{dist}\left(Y_{1}, Y_{2}\right) \geqq 2 \text {. }
$$

(B) Deterministic Bounds. The random variables $s_{n Y}$ will be deterministically "small," but, as explained above, the smoothness enters in these bounds in a nontrivial way. It will be convenient to introduce the following norms on $s_{n}$ :

$$
\left\|s_{n Y}\right\|_{1}=\sum_{u \in \mathbf{Z}^{d}} \sup _{x \in \mathbf{u}} \sup _{\tilde{\xi}}\left|s_{n Y}(x, \tilde{\xi})\right| e^{d(u \cup \tilde{u} \cup Y)} A(\tilde{k})^{-1 / 3} .
$$

Let us explain the notation. First, $s_{n Y}(x, \tilde{\xi})$ is given by (45) applied to the $y$ variable. Remark that $\tilde{u}$ localizes the $y$ variable. Next, by $d(X)$, for $X$ a finite set of points in $\mathbf{R}^{d}$, we will denote the length of the shortest connected graph on $X$ and possibly other points. Finally, $A(k)$ describes the smoothness:

$$
A(k)=\prod_{\mu=1}^{3}\left[1+\left|k_{\mu}\right|\right]^{-1} .
$$

From now on we set $d=3$. For $d>3$ the exponent in (50) is different. While the flow of the disorder is easy to express for any $d$ (see (68) below), the UV singularities, controlled by powers of $A(k)$, tend to make formulas complicated in general dimension. The choice of the power $1 / 3$ in (50) is due to a scaling argument that will become clear in the proof of Lemma 5.1.

Now, in terms of (50) and (51), we have

$$
\left\|s_{n Y}\right\|_{1} \leqq \delta_{n Y} \equiv \begin{cases}L^{-\alpha|Y|)} \delta_{n}^{3 / 2} & |Y|>1 \\ \delta_{n} & |Y|=1\end{cases}
$$

and

$$
\begin{gathered}
\delta_{n}^{2}=L^{-(1-2 \alpha) n} \delta_{0}^{2}, \\
\delta_{0}^{2}=\varepsilon^{2} \cdot K
\end{gathered}
$$

with $K$ large and $\alpha$ small.

Let us understand what $(52,53)$ mean. $s_{n}$ is the main part of the randomness in scale $L^{n}$, and this part will be deterministically bounded by $(52,53)$. In particular,

$$
\left|s_{n Y}(x, \tilde{\xi})\right| \leqq \delta_{n Y} e^{-d(x \cup \tilde{u} \cup Y)} A(\tilde{k})^{1 / 3} .
$$

For general $d$, we have, in (54), $\sim L^{-n(d-2)}$ (up to $\alpha$ ), but the Fourier transform has 
poor falloff: (56) implies, using Schwartz' inequality and Plancherel theorem:

$$
\int_{\tilde{\mathbf{u}}}\left|s_{n Y}(x, y)\right| d y \leqq \delta_{n Y} e^{-d(x \cup \tilde{u} \cup Y)}\left(\sum_{k} A(k)^{2 / 3}\right)^{1 / 2}
$$

and, from (51) and (44),

$$
\left(\sum_{k} A(k)^{2 / 3}\right)^{1 / 2} \sim C L^{n / 2}
$$

Thus, from (54) and (58),

$$
\delta_{n}^{3 / 2} L^{n / 2}=\mathcal{O}\left(L^{-\left(\frac{1}{4}-\frac{3 \alpha}{2}\right) n}\right)
$$

i.e. (59) is small, but

$$
\delta_{n} L^{n / 2} \sim L^{\alpha n}
$$

which is large.

$(52,53)$ thus mean that, except for $|Y|=1$, the $s_{n Y}$ have $L^{1}$-norms in the second variable tending to zero as $n \rightarrow \infty$, uniformly in $x$. Consider then $|Y|=1$. These are the terms we already addressed in the linear analysis and which, as we saw, did not contract with $n$, pointwise in $x$ and $y$. This is reflected in the blow-up of our bound (60). A more careful analysis will show that

$$
\int_{v}\left|s_{n u}(x, y)\right| d y \leqq e^{-d(x \cup u \cup v)} \cdot \begin{cases}\delta_{n}^{\alpha} & |u-v| \geqq 2 \\ \delta_{0}^{\alpha} & |u-v|<2,\end{cases}
$$

i.e. the leading term in $s_{n}$ is $s_{n u}(x, y)$ with $y$ near $u$ and this has an integral that stays small for all $n$, but will not contract with $n$. Note that these would be the terms in (31), linear in $b$, localized near the end point of the walk. The effective transition probability densities $p_{n}$ thus have a stochastic part that becomes small with $n$, when smeared with smooth functions, but stays just bounded when smeared with $L^{\infty}$ functions.

(C) Stochastic Bounds. The $s_{n}$ are random variables, deterministically bounded by $(52,53)$ and $(61)$. We need also bounds for them as random variables. The reason is very simple. As argued before, the variance of $b_{n}$ should contract with $n$ (modulo the smoothness problems). However deterministically the $b_{n}$ may be large: this we saw already when studying the linear RG [see (31)]. Actually, (33), improved by the $L^{-1}$ discussed in (36), says that $b_{1}$ is roughly a sum of $L^{d} b^{\prime} s$, multiplied by $L^{1-d}$. Hence, $b_{1}$ may be as large as

$$
\left|b_{1}(x, y)\right| \geqq C L \delta_{0}
$$

if $|b|<\delta_{0}$.

Therefore, $R s_{n}$ will not satisfy (53), with $n \rightarrow n+1$, and we want to show that the event that (53) is violated is unlikely. $s_{n+1}$ will then be the random variable conditioned on the likely event, and the rest is put into $r_{n+1}$. On the other hand, (52) will hold deterministically, due to the exponential decay in (38), and the rescaling involved in the renormalisation group transformation (see Proposition 5.2).

We will prove bounds for the generating function of $s_{n}$. Let

$$
\phi_{n}=\operatorname{Res}_{n} \text { or } \operatorname{Ims}_{n}
$$


and

$$
C_{u}\left(x_{1}, \xi_{2}\right)=e^{-2 d\left(x_{1} \cup u_{2} \cup u\right)} A\left(k_{2}\right)^{1 / 3}
$$

for $\xi_{2}=\left(u_{2}, k_{2}\right)$. Then

$$
E\left(\exp \sum_{k_{2}} f\left(k_{2}\right) \phi_{n u}\left(x_{1}, \xi_{2}\right)\right) \leqq \exp \varepsilon_{n}^{2}\left(\sum_{k_{2}}\left|f\left(k_{2}\right)\right| C_{u}\left(x_{1}, \xi_{2}\right)\right)^{2}
$$

with

$$
\varepsilon_{n}^{2}=L^{-(1-\alpha) n} \varepsilon^{2} .
$$

Note that (65) means that the variance of $s_{n}$ is

$$
E \phi_{n u}\left(x_{1}, \xi_{2}\right)^{2} \leqq C \varepsilon_{n}^{2} e^{-2 d\left(x_{1} \cup u \cup u_{2}\right)} A\left(k_{2}\right)^{2 / 3}
$$

which will ensure that $R s_{n}$ violates (53) with small probability, using the fact that we have $\alpha$ in (66) and $2 \alpha$ in (54).

For general $d,(66)$ is replaced by

$$
\varepsilon_{n}^{2}=L^{-(d-2-\alpha) n} \varepsilon^{2} .
$$

The power $\frac{2}{3}$ in (67) is consistent with what we said before about $\varepsilon_{n}$ : (67) yields an $L^{\infty}$-bound in $y$, on $s_{n}(x, y)$,

$$
\left|\sum_{k} A(k)^{1 / 3}\right| \sim C L^{2 n}
$$

killing all the contraction (and more!) in $\varepsilon_{n}^{2}$.

\section{The Large Fields}

We will now turn to the "large field" variables $r_{n}$. As we already mentioned, these arise because the expansion (28), which is used to localize $b_{1}$, is meaningless for $b$ not small enough. This is the problem of traps in our model which, in particular, give rise to the assumption (2.12) on the probability of the $p(x, y)$ 's being near zero. To see this, consider the following configuration: $p(x, y) \sim p(y, x) \sim 1$, $p(x, z), p\left(y, z^{\prime}\right) \sim 0, z \neq y, z^{\prime} \neq x$, and $p(z, x), p\left(z^{\prime}, y\right) \sim \frac{1}{2 d-1}$. The set $D=\{x, y\}$ is a trap: it is easy for the walk to enter $D$, but hard to leave. Note that such a configuration would be impossible in a symmetric model where $p(x, z)=p(z, x)$.

The condition (2.12) is now easy to understand: if a walk enters our trap, it will stay there for time $T$ such that $\left(1-\mathcal{O}\left(e^{-N}\right)\right)^{T} \sim 1 / 2 d$, i.e. $T \sim e^{N}$. In this time, without the trap, the walk would have diffused a distance $T^{1 / 2} \sim e^{N / 2}$. Hence, we want the mean distance between the traps be larger than this. If $P(N)$ is the probability

$$
P(N)=\operatorname{Prob}\left(p(x, y) \in \frac{1}{2 d}\left[e^{-N-1}, e^{-N}\right]\right),
$$

then we want

$$
P(N)^{-1 / d} \gg e^{N / 2}
$$

which is guaranteed by (2.12). Note that, for $\Gamma$ in (2.12) too small, we would expect the walk not to diffuse. 
We now face two problems. The first is how to separate the $s$ and $r$ variables from each other, i.e. how to localize the traps. The second problem concerns more complicated traps, namely the traps that can occur in arbitrary distance scales. Indeed, even if we started with a model where the random variables $|b(x, y)|<\delta_{0}$ for all $x, y$, large $b_{n}^{\prime} s$ will be generated, see (62), and, hence, arbitrary traps.

The separation (46) is straightforward in the first RG step. We define the "trapping region"

$$
\begin{gathered}
D=\left\{x:|b(x, y)|>\delta_{0},\right. \text { some } \\
y,|y-x|=1\}
\end{gathered}
$$

and simply put

$$
\begin{gathered}
s(x, y)=b(x, y) \chi(x \notin D), \\
l(x, y)=b(x, y) \chi(x \in D) .
\end{gathered}
$$

Next, we compute

$$
R s=\sum_{Y}(R s)_{Y}
$$

using the expansion (28) and look for a region $R \subset \mathbf{Z}^{d}$, where the bound (53) is violated for $R s$ (and $n=1$ ). We then define, roughly, the trapping region $D_{1}$, on scale 1 as

$$
D_{1}=L^{-1} D \cup R
$$

where $L^{-1} D$ is the rescaled $D$ set:

$$
L^{-1} D=\left\{x \in L^{-1} \mathbf{Z}^{d} \mid L x \in D\right\}
$$

(see Sect. 4 for precise definitions). $r_{1}$ will then have an expansion

$$
r_{1}(x, y)=\sum_{Y} r_{1 Y}(x, y)
$$

We will need two kinds of bounds for $r_{1 Y}$ :

(A) Lower Bounds for $p_{n}$. This is the crucial bound: the inductive, scale $L^{n}$, version of (2.12). This will tell us that a strong trap is unlikely on all scales. We introduce random variables, "the trapping density variables" $N_{n u}$ for $u \in \mathbf{Z}^{d}$. For $n=0$,

$$
\begin{gathered}
u \in D, \quad N_{0 u}=\min \left\{k \in(1-2 \alpha) \mathbf{N}\left|p(u, v)>L^{2-k} \forall v,\right| u-v \mid=1\right\}, \\
u \notin D, \quad N_{0 u}=-\infty .
\end{gathered}
$$

In this definition, 2 and $\alpha$ are put for bookkeeping purposes.

Now, $N_{n}, n \geqq 1$, is defined recursively, in terms of $N_{n-1}$ and new traps, coming from the region $R$, where $R s_{n-1}$ violates the bound (53). Rather than spell out this recursion in detail here, we explain its main features (see Sect. 6 for details). Thus, consider the typical event, where there is a single trap, say at the origin, and no others within a distance $e^{N_{00} \Gamma}$. Hence, the probability of escaping from 0 is

$$
\sum_{|y|=1} p(0, y) \geqq L^{2-N_{00}} \text {. }
$$


In the next scale, in case no new traps are created at $\mathbf{0}\left(=1\right.$-block in $L^{-1} \mathbf{Z}^{d}$ at 0$)$, the probability of escaping from 0 is given in terms of the original walk, in time $L^{2}$ :

$$
\min _{x \in \mathbf{0}} \int_{\mathbf{0} \mathbf{c}} p_{1}(x, y) d y=\sum_{|\omega|=L^{2}} p(\omega) \geqq C L^{2} L^{2-N_{00}}
$$

since we have $\mathcal{O}\left(L^{2}\right)$ times to exit the trap. Of course, (79) holds only when the righthand side is much smaller than 1, since the left-hand side is less than 1. Here, we only show how the flow of the large field goes; for precise bounds, see Sect. 8 .

Thus

$$
(79) \geqq L^{2-N_{00}+(2-2 \alpha)},
$$

i.e. the "trap strength" at 0 went down roughly by 2 units. This, of course, is nothing but a recursive way to see that, once we "wait" long enough, the trap is harmless, as discussed above. Thus, ignoring new traps, and possible old traps nearby, we may put

$$
N_{10}=N_{00}-(2-2 \alpha)
$$

whereby, for $\Gamma$ large,

$$
\begin{aligned}
\operatorname{Prob}\left(N_{10}=N\right) & \sim L^{d} \operatorname{Prob}\left(N_{00}=N+2-2 \alpha\right) \\
& \sim \mathrm{e}^{-N \Gamma} e^{-\Gamma(2-2 \alpha)}
\end{aligned}
$$

and, iterating this,

$$
\operatorname{Prob}\left(N_{n 0}=N\right) \sim e^{-(2-2 \alpha) n \Gamma} e^{-N \Gamma} .
$$

Once $N_{n u} \sim 0$, this will signal the absence of a trap at $u$. This does not mean that we may completely forget about such traps yet. As random variables, the transition probabilities connecting to such $u^{\prime} s$ are not of the form $T_{n}+s_{n}$, with $s_{n}$ small in the sense discussed above. We then proceed as follows: Note first that, starting with $N_{00} \neq 0, N_{n 0}$ becomes zero much before (84) gets any significant corrections, i.e. much before the $L^{\text {nd }}$ box at 0 is likely to contain any other $u$ with $N_{0 u} \neq 0$ or new traps generated by $R s$. This was the argument leading to (69) [see also (87) below]. Therefore, we may "wait" much longer still. It is easy to see what this means, if we go back to the starting scale. Then $r_{n}(x, y)$ is collecting walks, in time $L^{2 n}$, from $L^{n} x$ to $L^{n} y$, which visit 0 , our trap. Since there are no other traps, these are roughly free walks. Therefore, $r_{n}(x, y)$ becomes very small unless $y$ is very close to 0 (the end point problem discussed above). Roughly, for $x$ near 0 ,

$$
\int_{0}\left|r_{n}(x, y)\right| d y \sim L^{-\left(n-n^{\prime}\right) d}
$$

where $n^{\prime}$ is the scale s.t. $N_{n^{\prime} 0} \sim 0$, i.e., see (81),

$$
n^{\prime} \sim N_{00} / 2 .
$$

Thus, (85) becomes $\mathcal{O}\left(\varepsilon_{n}\right)$, i.e. of the order of the (variance) $)^{1 / 2}$ of $s_{n}$ when $d\left(n-n^{\prime}\right)$ $\sim \frac{1}{2} n$, i.e. when

$$
n=\mathcal{O}(1) N_{00} .
$$

At this point, $r_{n}(x, y)$ satisfies similar bounds as the $s_{n}$ and may be absorbed to them. Our original trap has disappeared (the estimates above will be slightly modified, since we want $r_{n}$ to satisfy the Fourier bounds $(52,53)$, see Sect. 7). From 
the argument above, see (84), we learned that, throughout the iteration

$$
\operatorname{Prob}\left(u \in D_{n}\right) \leqq e^{-c n \Gamma}
$$

with stronger bounds if $N_{n u}$ is large. Equation (86) will not be modified much by the traps in higher scales. These are generated when $R s_{n}$ violates (53), which has probability, using $(65)$

$$
\exp \left(-c \delta_{n}^{2} / \varepsilon_{n}^{2}\right)<\exp \left(-c L^{\alpha n} \delta_{0}^{2} / \varepsilon_{0}^{2}\right)
$$

which is super-exponential in $n$, in contrast to (86). To summarize: we need to keep lower bounds for $p_{n}$ in regions where $N_{n u}>0$. These are, when the right-hand side is small, of the form,

$$
\min _{|u-v|=1} \min _{\tilde{x} \in \mathbf{u}} \int_{\mathbf{v}} d \tilde{y} p_{n}(\tilde{x}, \tilde{y}) \geqq L^{\left(2-N_{n u}\right)} .
$$

(B) Upper Bounds for $p_{n}$. Two sorts of upper bounds need to be proven for the $r_{n Y}$ of (75) with $1 \rightarrow n$.

First, if $Y \cap\left\{u \mid N_{n}(u)>0\right\} \neq \phi$ there are "real" traps in $Y$. $r_{n Y}(x, y)$ will have exponential fall-off

$$
e^{-d(x \cup y \cup Y ; D)},
$$

where $d(X ; Y)$ denotes the length of the shortest graph on $X$ and possibly other points, which becomes connected when each connected component of $Y$ is shrunk to a point. This is obvious: outside the traps, $r_{n}$ should behave as $s_{n}$. See Sect. 6 for details. Secondly, it is convenient to let $N_{n u}$ become negative, after it reached the value zero, by the same iteration (81):

$$
N_{n+1,0}=N_{n 0}-(2-2 \alpha)
$$

(again under the assumption that traps are isolated, which is likely). Then, $r_{n Y}$ will carry factors of $L^{N_{n 0}}$ and $e^{-d(x \cup y \cup Y)}$, and will be absorbed into $S_{n Y}$ once these factors are small enough. Due to the Fourier transforms, this is slightly tedious, albeit straightforward, and we refer the reader to Sects. 6 and 7.

\section{Outline of the Paper}

The goal of Sect. 4 is still to explain the method: we perform explicitly the first RG step, but do not prove anything, since the propositions stated there are special cases of Proposition 6.1.

In Sect. 5, we do two kinds of estimates: first, we prove all the necessary properties of $T_{n}$, defined in (14). Then, we get the deterministic and probabilistic bounds on $s(x, y)$ (see point 3 above).

Sections 6 and 7 are devoted to the upper bounds on $r_{n}$. These bounds will be expressed in terms of the random variables $N$; The net result being that $r_{n Y}=0$ whenever $N_{n u}=-\infty$, for all $u \in Y$. So, if, in some region, we have $N_{n u}=-\infty$, we know that $b=s$ there, and we may use the deterministic bounds (52,53). In Sect. 6, we get $L^{1}$ bounds on $r_{n Y}$. Once these bounds are small enough, we show, in Sect. 7, using the arguments that led to (85), that a few more iterations produce Fourier bounds like $(52,53)$. Then we may, in the next step, add $r_{n Y}$ to $s_{n Y}$, and set the new $r_{n Y}$ 
equal to zero. These sections are the most technical of the paper; to shorten Sect. 6, we have put most of the proofs in an appendix.

An essential ingredient of Sect. 6 consists of an upper bound on $r(x, y)$ for $x$ inside a trap. However, this upper bound follows itself from a lower bound on the probability of escaping from a trap, roughly given by (88). This lower bound is proven in Sect. 8. This is the part of the paper where a non-perturbative argument is needed, since traps can be arbitrarily bad.

The net result of Sects. 6-8 are bounds on the large fields in terms of the trap strength variables $N_{n u}$. These satisfy a recursion relation given by $(6.10-6.14) . N_{n u}$ decreases, see (90), unless several $N_{n u}$ 's fall in the same $L$-cube, or new large fields are produced. Moreover, when $N_{n u}$ becomes sufficiently negative so that the corresponding $r_{n u}$ 's can be reabsorbed into $s$, we set the new $N_{n u}=-\infty$. It remains to show that the event $N_{n u}=-\infty$ is likely. This is done in Sect. 9. The proof is easy to understand: the bound (87) shows that new large fields are increasingly unprobable. So, we have mostly to control the possibility that several $N_{n u} \neq-\infty$ fall in the same $L$-cube. This is where $\Gamma$ large in (2.12) is used. By (69), we see that, for $\Gamma$ large, the typical distances between large fields are so big that these will be reabsorbed in the small fields much before two of them have a significant probability to fall in the same $L$-cube.

Finally, in Sect. 10, we prove Theorems 1 and 2. Using the estimates of Sect. 9, we know that the event $b_{n}(x, y)=s_{n}(x, y)$, for all $x, y$ visited by the walk, is very probable for $n$ large enough. Then, we may use the deterministic bounds $(52,53)$ to prove convergence to the Wiener measure.

\section{Notation and Choice of Constants}

There are two notational difficulties in the paper: one is that we work with a "running" lattice $\left(L^{-n} \mathbf{Z}\right)^{d}$, and an ordinary $\mathbf{Z}^{d}$ lattice. The other has to do with a proliferation of constants and their relation to $\varepsilon_{0}$ and $\Gamma_{0}$ in Theorem 1.

To deal with the first problem, we shall generally denote by $x, y, z$ the variables in the running lattice and by $u, v, w$ those in $\mathbf{Z}^{d}$. This lattice is always imbedded in the finer one. Boldface symbols such as $\mathbf{u}$ or $\mathbf{x}$, denote the unit cube centered at $u$, or containing $x$, more precisely the intersection of that cube with the running lattice. Similarly, $\mathbf{Y}$, for $Y \subset Z^{d}$, is the corresponding union of unit cubes. Often, we shall not distinguish between a set of cubes and the corresponding union. Symbols such as $L u, L^{-1} u, L Y$ denote the sites or the sets rescaled in the indicated way [see (74)]. $\left[L^{-1} u\right]$ is the point in $\mathbf{Z}^{d}$ whose coordinates are the integer parts of those of $L^{-1} u$, and similarly for sets. We use the Euclidean distance, so that two cubes $\mathbf{u}, \mathbf{v}$ are adjacent iff they touch iff $|u-v|<2$ (in three dimensions). For $X, Y$ subsets of $\left(L^{-n} \mathbf{Z}\right)^{d}, d(X)$ is the length of the shortest connected graph whose set of vertices contains $X$, and $d(X ; Y)$ is defined similarly, except that the graph becomes connected when each connected component of $Y$ is contracted to a point. $|E|$ is the volume for a set in $\left(L^{-n} \mathbf{Z}\right)^{d}$, and the cardinality for one in $\mathbf{Z}^{d}$. For a constant $a$, we let $B(a)=\bigcup\{\mathbf{u}$, such that $d(u, 0) \leqq a\}$ and $E+B(a)$ denotes the obvious union.

For the second notational problem, we let $c, C$ denote a generic constant, so that $2 c<c$ is allowed. The point is that those constants are independent of our choice of the renormalisation scale $L$. It is convenient to introduce constants that depend on $L$ (but not on the scale $n$ ): $c(L)$ denotes a generic function of $L$. We shall also use specific constants, $L^{\alpha}, L^{\beta}, L^{\gamma}$, where we let $\gamma \ll \beta \ll \alpha^{2}$ and $\alpha \ll 1$. These 
relations will constantly be used without mentioning them: for example, we assume that (87) is small, and at the same time, we use $\delta_{n}^{3 / 2} \ll \varepsilon_{n}$, which holds for $\alpha$ small and $L$ large. Actually, we choose $L$ so large that $c \ll L^{\gamma}$ and $L^{p} \ll \exp \left(L^{\gamma}\right)$, for some large $p$. It is also convenient to call the original scale $n_{0}$, instead of 0 . Doing that, we may write $\delta_{0}^{2}=L^{-(1-2 \alpha) n_{0}}$ and $\delta_{n}^{2}=L^{-(1-2 \alpha) n}$, see $(54,55)$. Of course, with this choice, the running lattice becomes $\left(L^{-\left(n-n_{0}\right)} \mathbf{Z}\right)^{d}$. We write $n-n_{0}=\tilde{n}$.

The logic leading to the choice of $\varepsilon_{0}, \Gamma_{0}$ is as follows: First, pick $\alpha, \beta, \gamma$ as above. Next, choose $L$ large enough to control various constants. Then, take $n_{0}$ large (depending on $L$ ); this determines $\varepsilon_{0} . \Gamma_{0}$ is taken equal to $L n_{0}^{2}$, see Sect. 9 .

\section{The First RG Step}

We start from (3.70)-(3.71). The analysis of (3.72), i.e. the perturbative small field analysis is presented in Sect. 5. Here we just take as input the region $R$ where $\mathscr{R} s$ is "large," i.e. violates the bound (3.53). This is defined precisely in (5.90) and enters the definition of the new large field region, which is a union of $L^{-1}$ cubes.

$$
D_{1}=\left((\tilde{D} \cup R)+B\left(2 L^{y}\right)\right) \cup L^{-1}\left(D+B\left(L^{\alpha}\right)\right)
$$

with

$$
\tilde{D}=\left\{\mathbf{u}\left|u \in \mathbf{Z}^{d} ; \exists v ;\right| v-u|<2,| D \cap L \mathbf{v} \mid>L^{\beta}\right\} .
$$

Let us explain this complicated-looking definition. Our goal is to derive for

$$
b_{1}(x, y)=L^{3} \int d \omega[(T+b)(\omega)-E(T+b)(\omega)]
$$

with $d \omega=d \omega_{L x L y}^{L^{2}}$, a splitting into "small" and "large" fields, as in (3.71). $L \tilde{D}$ is a region where (or near to where) there is a relatively high density of traps $\left(\geqq L^{\beta-3}\right)$. In (1), we put a "corridor" of width $\sim L^{1+\gamma}$ around $L(\widetilde{D} \cup R)$. The rest of $D$ is given a smaller corridor of width $\sim L^{\alpha}$. These corridors mean that a visit to $D$ starting from outside $L D_{1}$, i.e. $L x \notin L D_{1}$, will be quite unlikely.

We write

$$
b_{1}(x, y)=s_{1}(x, y)+e_{1}(x, y), \quad x \notin D_{1},
$$

where $e_{1}$ collects such visits to $D$ (and $L R$ ), see below. We expect $e_{1}$ to be small due to small hitting probabilities, although not necessarily as small as $s_{1} . e$ therefore denotes "expandable."

For $x \in D_{1}$ we write

$$
b_{1}(x, y)=s_{1}(x, y)+e_{1}(x, y)+l_{1}(x, y),
$$

where $l_{1}$ will be the new large field variable $(e+l$ was called $r$ in Sect. 3). It collects the trapped walks; as a random variable it is dependent on $b$ in a neighborhood of the connected component of $L D_{1}$ to which $L x$ belongs. $e_{1}$ collects visits to traps not localized to one component and therefore expandable, because, due to the corridors, hitting probabilities are small.

We now define $e_{1}$ and $l_{1}$. We expand $s$ in (3) $[b=s+l$, where $l$ in the first inductive step is given by $r$ defined in Eq. (3.71)] and localize the walks according to visits to $D$. Let first $x \notin D_{1}$. For each $v \in D$ we define a set $W(v)$ :

(a) if $v \in L \tilde{D}, W(v)=L D_{1}(v)$, the connected component of $L D_{1}$ containing $v$, (b) if $v \in D \backslash L \tilde{D}, W(v)$ is the connected component of $D+B\left(L^{\alpha}\right)$ containing $v$. 
We decompose the walks $\omega$ in (3) according to the first visit to $D$, at $v_{1}$, the first exit of $W\left(v_{1}\right)$ at $u_{1}$, the next return to $D$ at $v_{2}$ and so on. The reason for defining $W(v)$ as we did, instead of just as connected components of $L D_{1}$ is that, because of the corridors, a small piece of $D$ may fall inside a large component of $L D_{1}$. Inside $W(v)$, we just resum all walks and keep crude bounds. We thus get

$$
\begin{aligned}
\int d \omega[(T+b)(\omega)-(T+s)(\omega)]= & \sum_{k \geqq 2} \sum_{t_{\imath} t_{t}^{\prime}} \sum_{v_{\imath} \in D} \sum_{u_{i} \notin W_{i}} \prod_{i=1}^{k}(T+s)_{D^{c}}^{t_{i}}\left(u_{i-1}, v_{i}\right) \\
& \times \prod_{i=1}^{k-1}(T+b)_{W_{i}}^{t_{i}^{\prime}}\left(v_{i}, u_{i}\right)-(b \rightarrow s),
\end{aligned}
$$

where $u_{0}=L x, v_{k}=L y$, and $W_{i}=W\left(v_{i}\right)$. The $t_{i}, t_{i}^{\prime}$ satisfy $\sum\left(t_{i}+t_{i}^{\prime}\right)=L^{2}, t_{i}, t_{i}^{\prime} \geqq 1$, $i=1, \ldots, k-1$, and $t_{k} \geqq 0$. $\sum^{\prime}$ refers to the $t_{k}=0$ case: then, $v_{k}=u_{k-1}=L y$ and the constraint $u_{k-1} \notin W_{k-1}$ is not applied (Ly may belong to $W_{k-1}$ ). We also introduced the notation

$$
(T+s)_{D^{c}}^{t}(u, v)=\int d \omega_{u v}^{t}(T+s)(\omega) \prod_{i=1}^{t-1} \chi\left(\omega(i) \in D^{c}\right)
$$

In (6) we subtracted on the left-hand side the pure $s$ contribution (which is treated perturbatively in Sect. 5). $(b \rightarrow s)$ means that we have the same expansion but with $b$ replaced by $s$. In (6), the factors $(T+b)_{W}^{t^{\prime}}$ are localized, as random variables, in $W$ (see definition below). But $(T+s)_{D^{c}}^{t}$ is not localized because of the characteristic function on $D^{c}$, and because of $s$. Since $s(x, y) \chi\left(x \in D^{c}\right)=s(x, y)$ [s vanishes on $D$, see (3.71)] we have

$$
(T+s)_{D^{c}}^{t}(u, v)=\sum_{k \geqq 1} \sum_{n_{i} \geqq 0} \sum_{\left|x_{i}-y_{i}\right|=1} \prod_{i=1}^{k} T_{D^{c}}^{n_{i}}\left(x_{i-1}, y_{i}\right) \prod_{i=1}^{k-1} s\left(y_{i}, x_{i}\right),
$$

where $x_{0}=u, y_{k}=v$. The next thing to expand is the constraint on $D^{c}$ in $T_{D^{c}}^{n}$ in (8) (this is not a localized random variable!): write, in (7), $\chi\left(\omega(i) \in D^{c}\right)=1-\chi(\omega(i) \in D)$, and expand the product,

$$
T_{D^{c}}^{n}(x, y)=T^{n}(x-y)-\sum_{t_{1}=1}^{n-1} \sum_{v_{1} \in D} T^{t_{1}}\left(x-v_{1}\right) T_{D^{c}}^{n-t_{1}}\left(v_{1}, y\right) .
$$

Then, as in (6), we sum over the first exit from $W\left(v_{1}\right)$ :

$$
T_{D^{c}}^{m}\left(v_{1}, y\right)=\sum_{t_{1}^{\prime}} \sum_{u_{1} \in W_{1}} T_{W_{1} \cap D^{c}}^{t_{1}^{\prime}}\left(v_{1}, u_{1}\right) T_{D^{c}}^{m-t_{1}^{\prime}}\left(u_{1}, y\right)
$$

with $m=n-t_{1}$. Using (9), we iterate the procedure to get

$$
T_{D^{c}}^{n}(x, y)=\sum_{k \geqq 1} \sum_{t_{2} t_{i}^{\prime}} \sum_{v_{i} \in D} \sum_{u_{i} \notin W_{i}}^{\prime} \prod_{i=1}^{k} T^{t_{i}}\left(u_{i-1}-v_{i}\right) \prod_{i=1}^{k-1}\left[(-1) T_{W_{i} \cap D^{c}}^{t_{i}^{\prime}}\left(v_{i}, u_{i}\right)\right]
$$

with $v_{0}=x, v_{k}=y$, and the notation is as in (6).

Now we insert (10) into (8) and (8) into (6) to get

$$
\begin{aligned}
\int d \omega((T+b)(\omega)-(T+s)(\omega))= & \sum_{k \geqq 2} \sum_{t_{i}, t_{i}^{\prime}, a_{i}} \sum_{v_{i} u_{i}}^{\prime} \prod_{i=1}^{k} T^{t_{1}}\left(u_{i-1}-v_{i}\right) \\
& \times \prod_{i=1}^{k-1} Q_{a_{i}}^{t_{i}^{\prime}}\left(v_{i}, u_{i}\right)-(b \rightarrow s),
\end{aligned}
$$


where $a_{i}=1,2,3, t_{i} \geqq 0, i=1 \ldots k, t_{i}^{\prime} \geqq 1, i=1 \ldots k-1, \sum t_{i}+t_{i}^{\prime}=L^{2}$,

$$
\begin{gathered}
Q_{1}^{t^{\prime}}(v, u)=s(v, u) \cdot \delta_{t^{\prime}, 1}, \\
Q_{2}^{t^{\prime}}(v, u)=(T+b)_{W}^{t^{\prime}}(v, u), \\
Q_{3}^{t^{\prime}}(v, u)=-T_{W \cap D^{c}}^{t^{\prime}}(v, u) .
\end{gathered}
$$

The subtraction of $(b \rightarrow s)$ means that, for each term in (11),

$$
\exists i: a_{i}=2 \text {. }
$$

Finally, $\sum^{\prime}$ means $v_{i} \in D, u_{i} \notin W\left(v_{i}\right)$, for $a_{i} \neq 1$, unless $i=k-1$ and $t_{k}=0$, in which case $u_{k-1}=L y$.

The final rewriting of our expansion deals with these constraints in $\Sigma^{\prime}$

$$
\begin{gathered}
(11)=\sum_{k \geqq 2} \sum_{t_{i}, t_{i}, a_{i}, b_{i}} \sum_{\left(W_{i}\right)_{i}: a_{i}=2,3} \sum_{u_{i}, v_{i}} \prod_{i: a_{i}=2,3}^{\prime}\left(\chi\left(v_{i} \in W_{i} \cap D\right) \chi\left(u_{i} \notin W_{i}\right) \chi\left(W_{i} \in E_{b_{l}}\right)\right) \\
\times \prod_{i=1}^{k} T^{t_{i}}\left(u_{i-1}-v_{i}\right) \prod_{i=1}^{k-1} Q_{a_{i}}^{t_{i}}\left(v_{i}, u_{i}\right)-(b \rightarrow s) .
\end{gathered}
$$

We have the same notation as before. The sum over $W$ runs over all connected subsets of $\mathbf{Z}^{d}$. The sets $E_{b}$ are

$$
\begin{gathered}
E_{1}=\{W \mid \exists v \in D \backslash L \tilde{D} ; W=W(v)\}, \\
E_{2}=\{W \mid \exists v \in L \tilde{D}, W=W(v)\},
\end{gathered}
$$

where $W(v)$ has been defined in (a) and (b) above. Now the $u, v$ sums are unconstrained, the previous restrictions being in the characteristic functions. $\Pi^{\prime}$ means that there is no constraint on $u_{i} \notin W_{i}$ if $i=k-1$ and $t_{k}=0\left(u_{k-1}=L y\right.$ then). The point of (16) is that the summand is now a localized random variable.

Definition. A function $F(\mathbf{b})$ is a random variable localized in the set $S \subset L^{-(\tilde{n})} \mathbf{Z}^{d}$ at scale $L^{\tilde{n}}\left(\tilde{n}=n-n_{0}\right)$, if $F(\mathbf{b})$ is measurable with respect to the $\sigma$-algebra generated by

$$
\left\{b(x, y) \mid d\left(\left[L^{-\tilde{n}} x\right], S\right) \leqq 6 L^{\gamma}\right\} .
$$

Lemma 1. The summand in (16) is localized in

$$
\cup_{i} v_{i} \cup\left(\cup_{i} W_{i}+B\left(3 L^{1+\gamma}\right)\right)
$$

at scale 1.

Proof. $Q_{1}(v, u)$ is localized in $v$ and $Q_{2}(v, u)$ in $W(v) ; \chi\left(W \in E_{b}\right)$ is localized, at scale 1 , in

$$
W+B\left(3 L^{1+\gamma}\right) \text {. }
$$

Indeed, consider $b=2$, which is the most delocalized. $W \in E_{2}$ means that $W$ is a connected component of $L D_{1}$ intersecting $\widetilde{D}$. This gives a constraint, due to (1), that

$$
\operatorname{dist}(L(\widetilde{D} \cup R) \backslash W, W) \geqq 2 L^{1-\gamma} .
$$

From (2), we get another constraint, up to a distance $L$ from $L D_{1}$, i.e. (21) holds.

We can now, using (4) and (16), define $e_{1}$. First, we set

$$
L^{3} \int d \omega(T+s)(\omega) \equiv R s=s_{1}+\Delta e_{1},
$$


where $\Delta e_{1}=\sum_{Y} \Delta e_{1, Y}$ collects the terms for which (3.53) is violated, i.e. the $R$-contribution. This is defined and analyzed in Sect. 5 (see Proposition 5.5). Next, we collect terms in (16),

$$
L^{3} \int d \omega[(T+b)(\omega)-(T+s)(\omega)]=\sum_{Y} \tilde{e}_{Y}
$$

with

$$
Y=\left[L^{-1}\left(\cup_{i} \mathbf{v}_{i} \cup_{i} W_{i}\right)\right],
$$

and put

$$
e_{1 Y}=\tilde{e}_{Y}+\Delta e_{1 Y}-\tilde{E} \tilde{e}_{Y}
$$

We have

Proposition 1. Let $x \notin D_{1}$. Then $e_{1 Y}$ is localized in $Y$ at scale $L$ and satisfies

$$
e_{1 Y}=\varepsilon_{Y}+E_{Y}
$$

with

$$
\begin{gathered}
\sum_{Y} \int d y\left|\varepsilon_{Y}(x, y)\right| e^{d(x \cup y \cup Y)} \leqq L^{-\alpha / 2}, \\
\sum_{Y} \int d y\left|E_{Y}(x, y)\right| e^{d\left(x \cup y \cup Y ; D_{11 \cap Y}\right)} \leqq e^{-L^{\gamma}},
\end{gathered}
$$

and

$$
E_{Y}=0 \quad \text { if } \quad Y \cap \tilde{D}=\phi
$$

where

$$
D_{1 l}=(\tilde{D} \cup R)+B\left(2 L^{\gamma}\right) .
$$

Proposition 1 will be proved in Sect. 6 (it is a special case of Proposition 6.1), but note that the localization follows from Lemma 1. The bounds (27) and (28) are easy to understand too. $\varepsilon_{Y}$ collects terms in (16) with $b_{i}=1 \quad \forall i$, i.e. no visits to the dense trapping region. For $x \notin L D_{1}$ the collar $L^{\alpha}$ in (1) guarantees smallness via a Green's function bound $\frac{1}{d(x, D)} \sim L^{-\alpha}$. $E_{Y}$ collects terms with $b_{i}=2$ for some $i$, i.e. with visits to $\widetilde{D}$. Now the corridor $2 L^{1+\gamma}$ yields (28).

For $x \in D_{1}$ we again get (16), with the modification that $t_{1}=0$, i.e. $v_{1}=L x$, and $W_{1}$ is the connected component of $L D_{1}$ containing $L x$ (note that $L x \in L D_{1}$ need not belong to $D)$. Then, $l_{1}(x, y)$ collects terms with

$$
\left[L^{-1}\left(\cup_{i} \mathbf{v}_{i} \cup W_{i}\right)\right] C D_{1}(x)+B\left(2 L^{\gamma}\right),
$$

where $D_{1}(x)$ is the connected component of $D_{1}$ containing $x ; e_{1 Y}$ is as before for $\mathbf{Y} \not \subset D_{1}(x)+B\left(2 L^{\gamma}\right)$, and zero otherwise. Now $e_{1}=\varepsilon$ if $W_{1} \in E_{1}$ and $e_{1}=E$ if $W_{1} \in E_{2}$. Again, the bounds (27) and (28) follow since (31) means that the walks contributing to $\varepsilon$ or $E$ have to cross the corresponding corridors. For $l_{1}$, we will prove

Proposition 2. $l_{1}(x, y)=0$ unless $x \in D_{1}$, and is localized in $D_{1}(x)$ at scale L. It satisfies

$$
\int d y\left|l_{1}(x, y)\right| e^{d\left(y, D_{1}(x)\right)} \leqq C .
$$




\section{The Small Disorder Analysis}

In this section we analyze the RG map $R$ defined in (3.23) in the small field region. Thus, given a map

$$
s: L^{-\tilde{n}} \mathbf{Z}^{3} \times L^{-\tilde{n}} \mathbf{Z}^{3} \rightarrow \mathbf{R}
$$

$\left(\tilde{n}=n-n_{0}\right.$, and we suppress $n$ in $s_{n}$ and other variables, and put $s_{n+1}=s^{\prime}$ )

$$
R s: L^{-\tilde{n}-1} \mathbf{Z}^{3} \times L^{-\tilde{n}-1} \mathbf{Z}^{3} \rightarrow \mathbf{R}
$$

is given by

$$
(R s)\left(x^{\prime}, y^{\prime}\right)=L^{3} \int d \omega[(T+s)(\omega)-E(T+s)(\omega)]
$$

where

$$
d \omega=d \omega_{L x^{\prime} L y^{\prime}}^{L^{2}}
$$

is the path measure (3.4). $s^{\prime}$ is then given in terms of $R s$ by (4.22), see (67) below. The inductive properties of the $T$ and $s$ that we establish are the following.

Proposition 1. Under the assumptions of Theorem 2.1, $T_{n}$ may be expressed as

$$
T_{n}=\mathscr{T}_{n}+\tau_{n}
$$

where

$$
\begin{gathered}
\hat{\mathscr{T}}_{n}(k)=\hat{\mathscr{T}}_{0}\left(L^{-\tilde{n}} D_{n}^{1 / 2} k\right)^{L^{2 \tilde{n}}}, \\
\hat{\mathscr{T}}_{0}(k)=\frac{1}{3} \sum_{\alpha=1}^{3} \cos k_{\alpha},
\end{gathered}
$$

and

$$
\left|D_{n}-1\right| \leqq \text { const } \varepsilon^{2} \text {. }
$$

$\hat{\tau}_{n}$ is analytic in $\Sigma=\left\{k|| \operatorname{Im} k_{\mu} \mid<3\right\}$, and, for $k \in \Sigma$,

$$
\left|\hat{\tau}_{n}(k)\right| \leqq\left\{\begin{array}{l}
\delta_{n}^{3 / 2} A(k) \\
\delta_{n}^{3 / 2}(\bar{k} k)^{2}
\end{array} \text { for } \quad\left|\operatorname{Re} k_{\mu}\right|<\log L^{n} .\right.
$$

( $A(k)$ is defined in (3.51).)

Before we embark on the proof of Proposition 1, let us make the following technical remark. The nearest neighbour walk (2.6) has the property that the matrix $p$ has, apart from the eigenvalue 1 corresponding to constant functions, an eigenvalue -1 corresponding to the eigenfunction $e^{i \pi x \cdot(1,1,1)}$. This could make the analysis below more cumbersome and so we will circumvent the difficulty by doing the first step of the iteration as follows. We choose $L$ even, and first replace $p$ by $p^{2}$. Note, that for $p^{2}$ the eigenvalue 1 is degenerate, reflecting the fact that the Markov chain is now reducible. Indeed, $p^{2}$ maps the set of functions on the sublattice of $\mathbf{Z}^{d}$ generated by the vectors $\pm e_{\mu} \pm e_{v}$ into itself. This means that we may restrict our Fourier transform variables $k$ to "half" of the Brillouin zone, i.e., say, $\left|k_{1}\right|<\frac{1}{2} \pi L^{n}$. In particular, $\hat{\mathscr{T}}_{0}(k)$ in $(7)$ satisfies the bound $\left|\hat{\mathscr{T}}_{0}(k)\right|<e^{-c k^{2}}$ (for real $k$ and $n=0$ ) in that region leading to (21) below. Without further mention, all the momenta are assumed to lie in this reduced Brillouin zone. 
Most of the bounds for $s_{n}$ have been stated in Chap. 3. Let us gather them here: First, the Fourier bound

$$
\left\|s_{n Y}\right\|_{1} \leqq \delta_{n Y}
$$

[see (3.50) for the norm] and then the $L^{1}$-bound

$$
\int_{\mathbf{v}}\left|s_{n u}(x, y)\right| d y \leqq e^{-d(x \cup u \cup v)}\left\{\begin{array}{l}
\delta_{n}^{\alpha},|u-v| \geqq 2 \\
\delta_{0}^{\alpha},|u-v|<2,
\end{array}\right.
$$

and finally the stochastic bound (3.65):

$$
E\left(\exp \sum_{k_{2}} f\left(k_{2}\right) \phi_{n u}\left(x_{1}, \xi_{2}\right)\right) \leqq \exp \varepsilon_{n}^{2}\left(\sum_{k_{2}}\left|f\left(k_{2}\right)\right| C_{u}\left(x_{1}, \xi_{2}\right)\right)^{2},
$$

where $\phi=\operatorname{Res}$ or Im $s$. Actually, to prove (11) we need to introduce yet another pair of bounds, for the double Fourier transforms $s_{n}\left(\xi_{1}, \xi_{2}\right)$. Defining the norm

$$
\left\|s_{n Y}\right\|_{2}=\sum_{u_{1}} \sup _{k_{1}} \sup _{\xi_{2}}\left|S_{n Y}\left(\xi_{1}, \xi_{2}\right)\right| e^{d\left(u_{1} \cup u_{2} \cup Y\right)} A\left(k_{1}\right)^{-2 / 3} A\left(k_{2}\right)^{-1 / 3}
$$

we shall prove

$$
\left\|s_{n Y}\right\|_{2} \leqq \delta_{n Y}
$$

and furthermore that

$$
E\left(\exp \sum_{k_{1} k_{2}} f\left(k_{1}, k_{2}\right) \phi_{n u}\left(\xi_{1}, \xi_{2}\right)\right) \leqq \exp \varepsilon_{n}^{2}\left(\sum_{k_{1} k_{2}}\left|f\left(k_{1}, k_{2}\right)\right| C_{u}\left(\xi_{1}, \xi_{2}\right)\right)^{2},
$$

with

$$
C_{u}\left(\xi_{1}, \xi_{2}\right)=e^{-2 d\left(u \cup u_{1} \cup u_{2}\right)} A\left(k_{1}\right)^{2 / 3} A\left(k_{2}\right)^{1 / 3} .
$$

Equations (14)-(16) are needed to control convolutions $s * s$, for the proof of (11).

Given (5)-(16) for $T_{n}, s_{n}$, we now proceed to prove them for $T^{\prime} \equiv T_{n+1}, s^{\prime}=s_{n+1}$. Proof of Proposition 1. Separate first the s-contribution to $T^{\prime}$ :

and expand $s$

$$
T^{\prime}=L^{3} \int d \omega E(T+b)(\omega) \equiv L^{3} \int d \omega E(T+s)(\omega)+T_{3}
$$

$$
\begin{aligned}
L^{3} \int d \omega E(T+s)(\omega) & =L^{3} T^{L^{2}}\left(L x^{\prime}-L y^{\prime}\right)+L^{3} \sum_{\underline{n}} \int d \omega E \prod_{i=1}^{k} T^{n_{i}} S^{m_{i}} \\
& \equiv T_{1}\left(x^{\prime}-y^{\prime}\right)+T_{2}\left(x^{\prime}-y^{\prime}\right)
\end{aligned}
$$

with $\sum m_{i} \geqq 1$ and obvious constraints for $\underline{n}, \underline{m}$. $T_{1}$ is a multiple convolution

$$
\widehat{T}_{1}(k)=\hat{T}\left(\frac{k}{L}\right)^{L^{2}}=\left(\hat{\mathscr{T}}\left(\frac{k}{L}\right)+\tau\left(\frac{k}{L}\right)\right)^{L^{2}} \equiv \hat{\mathscr{T}}\left(\frac{k}{L}\right)^{L^{2}}+\hat{t}\left(\frac{k}{L}\right) .
$$

Now

$$
\hat{\mathscr{T}}\left(\frac{k}{L}\right)^{L^{2}}=\hat{\mathscr{T}}_{0}\left(L^{-\tilde{n}-1} D_{n}^{1 / 2} k\right)^{L^{2(\tilde{n}+1)}}
$$

and $\hat{t}\left(\frac{k}{L}\right)$ is analytic for $k$ in $L \Sigma$. Since, for $k \in \Sigma$, (6) and (7) imply

$$
|\hat{\mathscr{T}}(k)| \leqq c e^{-c|k|^{2}},
$$


we get from (21) and (9) that, for $k \in \Sigma,\left|\operatorname{Re} k_{\mu}\right|<\log L^{n+1}$ for all $\mu$,

$$
|\hat{t}(k)| \leqq C L^{-2} \delta_{n}^{3 / 2}(\bar{k} k)^{2} \ll \delta_{n+1}^{3 / 2}(\bar{k} k)^{2}
$$

and, for $\left|\operatorname{Re} k_{\mu}\right|>\log L^{n+1}$ for some $\mu$,

$$
|\widehat{t}(k)| \leqq \sum_{m=1}^{L^{2}}\left(\begin{array}{l}
L^{2} \\
m
\end{array}\right)\left[\delta_{n}^{3 / 2} A\left(\begin{array}{l}
k \\
L
\end{array}\right)\right]^{m}(C L)^{-a\left(L^{2}-m\right)}
$$

with

$$
a=C n^{2}(\log L) L^{-2}
$$

Easily then ( $n>n_{0}$ is large),

$$
|\widehat{t}(k)| \leqq \frac{1}{3} \delta_{n+1}^{3 / 2} A(k) .
$$

Now, consider $T_{2}$ in (18). Since $s$ has poor falloff for large $k$ in both variables separately, we use the translation invariance of $T_{2}$ to combine the falloff from both of them:

$$
\widehat{T}_{2}(k)=\int_{0} d x \int d y T_{2}(x-y) e^{-i k(x-y)}=\sum_{u_{2}} \sum_{k_{1} k_{2}} K\left(k-k_{1}\right) K\left(k+k_{2}\right) T_{2}\left(\xi_{1}, \xi_{2}\right) e^{i k u_{2}},
$$

where $\xi_{1}=\left(k_{1}, 0\right), \xi_{2}=\left(k_{2}, u_{2}\right)$. In (26) and frequently below, we use the following easy identities for the Fourier transform (3.45):

$$
\begin{gathered}
\int d x f(x) g(x)=\sum_{\xi} \overline{f(\xi)} g(\xi), \\
f(\xi)=\int d q K(k-q) \hat{f}(q) e^{-i q u},
\end{gathered}
$$

with

$$
\int d q=(2 \pi)^{-3} \int_{\left[-L^{n} \pi, L^{n} \pi\right]^{3}} d^{3} q
$$

and

$$
K(q)=\prod_{\mu=1}^{3} \frac{\sin \left(\frac{1}{2} q_{\mu}\right)}{L^{n} \sin \left(\frac{1}{2} L^{-n} q_{\mu}\right)} .
$$

Thus, we need to estimate $T_{2}\left(\xi_{1}, \xi_{2}\right)$ in (26). $T_{2}$ is given by

with

$$
T_{2}=\sum_{Y} E \sigma_{Y}
$$

$$
\sigma_{Y}\left(x^{\prime}, y^{\prime}\right)=L^{d} \sum_{n}\left(T^{n_{1}} S_{Y_{1}} T^{n_{2}} \ldots T^{n_{k}} S_{Y_{k}} T^{n_{k+1}}\right)\left(L x^{\prime}, L y^{\prime}\right)
$$

and $Y=\left(Y_{1}, \ldots, Y_{k}\right) \subset\left(\mathbf{Z}^{3}\right)^{k}, n_{i} \geqq 0, k+\sum n_{i}=L^{2}$.

From (28),

$$
T^{n}\left(\xi_{1}, \xi_{2}\right)=\int d q K\left(k_{1}-q\right) K\left(k_{2}+q\right) \widehat{T}(q)^{n} e^{i q\left(u_{2}-u_{1}\right)} .
$$

By analyticity of $\hat{T}$ in $\Sigma$ and the bounds (9) and (21), upon shifting

$$
q \rightarrow q+i s\left(u_{2}-u_{1}\right)\left|u_{2}-u_{1}\right|^{-1}
$$

with $0 \leqq s \leqq 2$, we obtain $(n>0)$

$$
T^{n}\left(\xi_{1}, \xi_{2}\right) \leqq c e^{c n s^{2}} e^{-s\left|u_{1}-u_{2}\right|} I\left(k_{1}, k_{2}\right),
$$


where

$$
I\left(k_{1}, k_{2}\right)=\int d q A\left(k_{1}-q\right) A\left(k_{2}+q\right) A(q)^{(1+\alpha)} .
$$

We used

$$
\begin{gathered}
\left|K\left(q+i q_{0}\right)\right| \leqq C A(q), \\
\left|\widehat{T}\left(q+i q_{0}\right)\right| \leqq e^{c q_{0}^{2}}, \\
\left|\widehat{T}\left(q+i q_{0}\right)\right| \leqq C A(q)^{1+\alpha} \leqq a(q) .
\end{gathered}
$$

Here, we use part of $\delta_{n}^{3 / 2}$ in (9), and $|q| \leqq c L^{n}$ to get the extra $\alpha$, which will only be used later. Equations (37-39) hold for $\left|q_{0}\right| \leqq 2$. I can be bounded by Schwartz and

$$
\int A(k-q)^{2} A(q)^{t} d q \leqq C A(k)^{t}, \quad 0 \leqq t \leqq 2
$$

as

$$
I\left(k_{1}, k_{2}\right) \leqq C A\left(k_{1}\right)^{2 / 3} A\left(k_{2}\right)^{1 / 3} .
$$

Combining (35) and (41) with (14) and (13), and doing the $k$ sums $\left(|k| \leqq c L^{n}\right)$

$$
\sum_{k} A(k)^{2 / 3} A(k)^{1 / 3} \leqq C\left(\log L^{n}\right)^{d}
$$

we get

$$
\begin{aligned}
\left|\sigma_{Y}\left(\xi_{1}^{\prime}, \xi_{2}^{\prime}\right)\right| \leqq & C(L)\left(\prod_{i=1}^{k} \delta_{Y_{i}}\right)\left(C \log L^{n}\right)^{d k} \sum_{n} \prod_{i} e^{c n_{i} s_{i}^{2}} \sum_{u_{i} \in L \mathbf{u}_{i}} 1 A\left(k_{1}^{\prime}\right)^{2 / 3} A\left(k_{2}^{\prime}\right)^{1 / 3} \\
& \times \sum_{v} e^{-s_{1}\left|u_{1}-v_{1}\right|} e^{-d\left(v_{1} \cup v_{2} \cup Y_{1}\right)} \ldots e^{-s_{k+1}\left|v_{k+1}-u_{2}\right|}
\end{aligned}
$$

where we used the following identity: let

$$
f_{L}(x)=f(L x)
$$

then

$$
f_{L}\left(\xi^{\prime}\right)=L^{-d} \sum_{u \in L \mathbf{u}^{\prime}} e^{i \frac{k^{\prime}}{L}\left(u-L u^{\prime}\right)} f\left(u, \frac{k^{\prime}}{L}\right) .
$$

We will use (43) later in a more substantial way when we estimate $s_{Y}^{\prime}$, but now just take $s_{i}=\frac{1}{2}$ and note that $E s_{Y}=0$ (see Proposition 2 below) means only $k \geqq 2$ contribute to (32):

$$
\left|T_{2}\left(\xi_{1}^{\prime}, \xi_{2}^{\prime}\right)\right| \leqq \lambda_{n} e^{-\frac{L}{3}\left|u_{1}^{\prime}-u_{2}^{\prime}\right|} A\left(k_{1}^{\prime}\right)^{2 / 3} A\left(k_{2}^{\prime}\right)^{1 / 3}
$$

with

$$
\lambda_{n}=C(L)\left(\log L^{n}\right)^{c} \delta_{n}^{2} \ll \delta_{n}^{3 / 2} .
$$

Inserting (46) into (26) we get, for $k \in \frac{L}{4} \Sigma$,

$$
\left|\widehat{T}_{2}(k)\right| \leqq \lambda_{n} A(k) .
$$

Finally, we consider $T_{3}$ in (17). This is the trap-contribution:

$$
T_{3}=L^{3} \int d \omega E((T+b)(\omega)-(T+s)(\omega)) .
$$


In Sect. 8 we show that

$$
E\left(\sup _{x \in \mathbf{u}} \int_{\mathbf{v}}\left|r_{n}(x, y)\right| d y\right)^{k} \leqq e^{-|u-v|} e^{-c n \Gamma^{1 / 2}}
$$

for $k \leqq L^{2}$. Whereby, expanding $b=s+r$ in (49) and using (11) for the $L^{1}$-norm of $s$, and Schwartz inequality repeatedly for several $r$,

$$
\left|T_{3}\left(x_{1}^{\prime}-x_{2}^{\prime}\right)\right| \leqq e^{-\frac{L}{3}\left|x_{1}^{\prime}-x_{2}^{\prime}\right|} e^{-c n \Gamma^{1 / 2}}
$$

which, for $\Gamma$ large, gives, in $k \in \frac{L}{4} \Sigma$,

$$
\left|\widehat{T}_{3}(k)\right| \leqq \delta_{n}^{2} A(k) .
$$

$T_{2}$ and $T_{3}$ will "renormalize" the effective diffusion constant $D_{n}$. To do this, recall that

$$
\int T(x) d x=1=\int T^{\prime}(x) d x,
$$

whence $\hat{T}(0)=1=\hat{T}^{\prime}(0)$ and so

$$
\widehat{T}_{4}(0)=\widehat{T}_{2}(0)+\widehat{T}_{3}(0)=0 .
$$

By the isotropy assumption (P1) in Sect. 2,

$$
\partial_{\mu} \widehat{T}_{4}(0)=0, \quad \partial_{\mu} \partial_{\nu} \hat{T}_{4}(0)=2 \gamma \delta_{\mu \nu}
$$

for some $\gamma$. By Cauchy's theorem ( $u$ is a unit vector) and (48), (52),

$$
|\gamma| \leqq(4 \pi)^{-1} \int_{0}^{2 \pi}\left|\widehat{T}\left(e^{i \theta} u\right)\right| d \theta \leqq \lambda_{n}
$$

and, since $\partial_{\mu} \partial_{\nu} \partial_{\varrho} \widehat{T}_{4}(0)=0$ by isotropy, we get

$$
\left|\widehat{T}_{4}(k)-\gamma k^{2}\right|=\frac{1}{6}\left|\int_{0}^{1} d t\left(\frac{d^{4}}{d t^{4}} \widehat{T}_{4}(t k)\right)(1-t)^{3}\right| \leqq\left|\oint_{|t|=(\bar{k} k)^{\frac{-1}{2}}} d t t^{-5} \widehat{T}_{4}(t k)\right| \leqq \lambda_{n}(\bar{k} k)^{2}
$$

for $k \in \Sigma,\left|\operatorname{Re} k_{\mu}\right| \leqq \log L^{n+1}$, using (48) and (52). We may now put $(d=3)$

$$
D_{n+1}=D_{n}-2 d \gamma
$$

so, by (56)

$$
\left|D_{n+1}-D_{n}\right| \leqq \lambda_{n}
$$

and

$$
T^{\prime}=\mathscr{T}^{\prime}+t+T_{4}+r
$$

with

$$
|\hat{r}(k)|=\left|\hat{\mathscr{T}}_{0}\left(L^{-\tilde{n}-1} D_{n}^{1 / 2} k\right)^{L^{2(\tilde{n}+1)}}-\hat{\mathscr{T}}_{0}\left(L^{-\tilde{n}-1} D_{n+1}^{1 / 2} k\right)^{L^{2(\tilde{n}+1)}}\right| \leqq \lambda_{n} e^{-c \bar{k} k}
$$

Equations (61), (48),(52), and (25) give the first part of (9) for $n+1$, and (22) and (57) yield the second inequality in (9). Indeed, the quadratic part of $\hat{r}(k)$ is exactly cancelled by $\gamma k^{2}$, coming from (55). This explains the "renormalisation" of $D$. Equation (8) follows from (59). 
Definition of $s_{n+1}$. Let $s_{n}$ satisfy the bounds (10) and (14). $R s_{n}$ will violate these bounds with small probability. We want to define $s_{n+1}$ such that (10) and (14) are true again, for $n \rightarrow n+1$. It turns out that it suffices to make restrictions only in terms of the linearized RG map (3.31). Let us formalize this. We set

by

$$
\mathscr{L} S=\sum_{v^{\prime} \in \mathbf{Z}^{d}}(\mathscr{L} S)_{v^{\prime}}
$$

$$
(\mathscr{L} S)_{v^{\prime}}\left(x^{\prime}, y^{\prime}\right)=L^{3} \sum_{v \in L v^{\prime}} \sum_{t=0}^{L^{2}-1} \int d x d y T^{t}\left(L x^{\prime}-x\right) T^{L^{2}-t-1}\left(L y^{\prime}-y\right) S_{v}(x, y)
$$

and look where $\mathscr{L}_{S}$ violates (10) and (14). Let

$$
\chi_{v}=\chi\left(\left\|(\mathscr{L} s)_{v}\right\|_{i} \leqq \frac{1}{4} \delta^{\prime}, i=1,2\right)
$$

and set

$$
\varrho_{v}=(1-E)\left(\mathscr{L}_{s}\right)_{v} \chi_{v},
$$

where $(1-E)$ means that we subtract the average. Note, that $E \mathscr{L}_{S_{v}} \chi_{v} \neq 0$ is nonzero because of the $\chi_{v}$. Then,

$$
\left\|\varrho_{v}\right\|_{i}<\frac{1}{2} \delta^{\prime}
$$

and we define $\left(s^{\prime}=s_{n+1}\right)$

$$
s^{\prime}=\varrho+(R s-\mathscr{L} s)+\Delta s,
$$

where $\Delta s$ is the reabsorbed trap contribution

$$
\left\|(\Delta s)_{Y}\right\|_{i} \leqq \frac{1}{4} \delta^{\prime 3 / 2} L^{-\alpha|Y|},
$$

see Sect. 7. It is convenient to localize (67) in the following way. Consider Rs given by (3).

We expand both terms as in (18) and (31), to get

$$
R s=\sum_{Y}\left(\sigma_{Y}-E \sigma_{Y}\right) .
$$

We put

$$
(R s)_{v}=\sum_{Y}^{\prime}\left(\sigma_{Y}-E \sigma_{Y}\right) n(\underline{Y})^{-1}
$$

with $\cup Y_{i} \subset L \mathbf{v}$, or $Y=Y_{1}$, i.e. $k=1$ in (32) and $\operatorname{diam}\left(Y_{1}\right)<\frac{1}{4} L, Y_{i} \cap L \mathbf{v} \neq \phi$, i.e. we gather to $(R s)_{v}$, the local part, all $Y_{i}^{\prime}$ s that touch $L \mathbf{v}$ and are allowed to extend up to distance $\frac{L}{4}$ from $L v$. This will guarantee that $(R s)_{Y}$ for $|Y|>1$ will contract strongly. $n(Y)$ is the number of $L$-cubes for which $Y$ contributes to the sum. To complete the definition, we collect the rest of $\underline{Y}$ to $(R s)_{Y}$ :

$$
(R s)_{Y}=\sum_{Y}^{\prime \prime}\left(\sigma_{Y}-E \sigma_{Y}\right)
$$

with $Y=\left[L^{-1} \cup Y_{i}\right]$. $s_{Y}^{\prime}$ is now defined. We proceed to discuss its properties.

Proposition 2. $s_{Y}^{\prime}$ is localised in $\mathbf{Y}$. Moreover, $s_{Y_{1}}^{\prime}, s_{Y_{2}}^{\prime}$ are independent, if $d\left(Y_{1}, Y_{2}\right) \geqq 2$. Also, $E s_{Y}^{\prime}=0$ and

$$
\int s_{Y}^{\prime}(x, y) d y=0
$$


Proof. By induction, (71) satisfy the claims, as do the $\left(\mathscr{L}_{S}\right)_{v^{\prime}}$ and $\chi_{s}^{\prime}$. For (70), let $\left|v_{1}-v_{2}\right| \geqq 2$. Then, if $Y_{1}$ contributes to $s_{v_{1}}$ and $Y_{2}$ to $s_{v_{2}}, d\left(Y_{1}, Y_{2}\right)>\frac{L}{2}$ and the localisation and the independence hold for (70). $\Delta s_{Y}$ are constructed (see Sect. 7) such that the claims of Proposition 2 hold. Then $E s_{Y}^{\prime}=0$ by construction, and (72) holds since by induction it is true for $s_{Y}$ and then, using $\int T(x) d x=1$, it holds for $\sigma_{Y}$ and $\varrho$.

Proposition 3. We have the bound

$$
\left\|s_{Y^{\prime}}^{\prime}\right\|_{i} \leqq \delta_{Y}^{\prime} .
$$

Proof. We use (43), where we insert $s_{i}=2\left(n_{i}+1\right)^{-1 / 2}$. (Recall: $s_{i} \in[0,2]$.) Using

$$
d\left(v_{i} \cup v_{i+1} \cup Y_{i}\right)=L d\left(\frac{v_{i}}{L} \cup \frac{v_{i+1}}{L} \cup \frac{1}{L} Y_{i}\right)
$$

and easy estimates, we get

$$
\left|\sigma_{Y}\left(\xi_{1}^{\prime}, \xi_{2}^{\prime}\right)\right| \leqq\left(\log L^{n}\right)^{k-1} L^{c k} \prod_{i=1}^{k} \delta_{Y_{i}} A\left(k_{1}^{\prime}\right)^{2 / 3} A\left(k_{2}^{\prime}\right)^{1 / 3} e^{-d\left(u_{1}^{\prime} \cup u_{2}^{\prime} \cup L^{-1} Y\right)} \prod e^{-\frac{1}{2} d\left(Y_{i}\right)}
$$

with $Y=\cup Y_{i}$.

Let first $\left|Y^{\prime}\right|>1$. Then, only $k>1$, or $Y_{1}$ with $\operatorname{diam}\left(Y_{1}\right) \geqq \frac{L}{4}$ contribute to (75), and, for $L$ large,

$$
\left|S_{Y^{\prime}}^{\prime}\left(\xi_{1}^{\prime}, \xi_{2}^{\prime}\right)\right| \leqq e^{-C L} L^{-\alpha \mid Y^{\prime}} \delta^{3 / 2} A\left(k_{1}^{\prime}\right)^{2 / 3} A\left(k_{2}^{\prime}\right)^{1 / 3} e^{-d\left(u_{1}^{\prime} \cup u_{2}^{\prime} \cup Y^{\prime}\right)} .
$$

We used $\left[L^{-1} Y\right]=Y^{\prime}$ to deduce

$$
d\left(u_{1}^{\prime} \cup u_{2}^{\prime} \cup L^{-1} Y\right) \geqq d\left(u_{1}^{\prime} \cup u_{2}^{\prime} \cup Y^{\prime}\right)-C
$$

and controlled the sums over $k$ and $Y_{i}$ by $e^{-\frac{1}{2} d\left(Y_{i}\right)}$ and powers of $\delta$. Equation (76) yields (73), $i=2$. For $\left|Y^{\prime}\right|=1,\left(R s-\mathscr{L}_{s}\right)_{v^{\prime}}$ is second order in $s_{v}$ or involves $s_{Y},|Y|>1$. Thus

$$
\left|\left(R s-\mathscr{L}_{S}\right)_{v^{\prime}}\left(\xi_{1}^{\prime}, \xi_{2}^{\prime}\right)\right| \leqq C(L) \delta^{3 / 2} A\left(k_{1}^{\prime}\right)^{2 / 3} A\left(k_{2}^{\prime}\right)^{1 / 3} e^{-d\left(u_{i}^{\prime} \cup u_{2}^{\prime} \cup v^{\prime}\right)} .
$$

Together with (66) and (68), (73) follows again, for $i=2$. For $i=1$ the analysis is parallel, starting from the bound (76) without $A\left(k_{1}^{\prime}\right)^{2 / 3}$ for $\sigma_{Y}\left(x_{1}^{\prime}, \xi_{2}^{\prime}\right)$.

Proposition 4. Equation (11) holds for $n \rightarrow n+1$.

Proof. Equation (68) and (78) yield

$$
\int_{\mathbf{u}^{\prime}}\left|\left(R s-\mathscr{L}_{s}+\Delta s\right)_{v^{\prime}}\left(x^{\prime}, y^{\prime}\right)\right| d y^{\prime} \leqq L^{-n \alpha} \delta_{n}^{\alpha} e^{-d\left(u^{\prime} \cup v^{\prime} \cup x^{\prime}\right)}
$$

by virtue of (3.59), for all $u^{\prime}, v^{\prime}$. Thus (79) is negligible and we turn to $\varrho$ in (67).

We decompose it as

$$
\varrho_{v^{\prime}}\left(x^{\prime}, y^{\prime}\right)=L^{3} \sum_{v \in L \mathbf{v}^{\prime}} \int d x T^{L^{2}-1}\left(L x^{\prime}-x\right) s_{v}\left(x, L y^{\prime}\right)+\varrho_{v^{\prime}}^{2}\left(x^{\prime}, y^{\prime}\right) \equiv \varrho_{v^{\prime}}^{1}+\varrho_{v^{\prime}}^{2} .
$$

In $\varrho^{2}$, a $T$ factor appears having $L y^{\prime}$ as its second argument, so we get, using (10),

$$
\int_{\mathbf{u}^{\prime}}\left|\varrho_{v^{\prime}}^{2}\left(x^{\prime}, y^{\prime}\right)\right| d y^{\prime} \leqq C \delta_{n} e^{-d\left(u^{\prime} \cup v^{\prime} \cup x^{\prime}\right)} .
$$


For $\varrho^{1}$, if $\left|u^{\prime}-v^{\prime}\right| \geqq 2$, we get

$$
\int_{\mathbf{u}^{\prime}}\left|\varrho_{v^{\prime}}^{1}\left(x^{\prime}, y^{\prime}\right)\right| d y^{\prime} \leqq \int d x e^{-\frac{3}{2}\left|x^{\prime}-\frac{x}{L}\right|} \sum_{u \in L \mathbf{u}^{\prime} v \in L \mathbf{v}^{\prime}} \delta_{n}^{\alpha} e^{-d(u \cup v \cup x)} \leqq e^{-C L} \delta_{n}^{\alpha} e^{-d\left(x^{\prime} \cup u^{\prime} \cup v^{\prime}\right)},
$$

where (11) was used. Thus, the first inequality in (11) follows for $n \rightarrow n+1$.

For the second inequality, let us denote by $\sigma_{u}$ the random variables

$$
\sigma_{u}(x, y)= \begin{cases}s_{u}(x, y), & y \in \mathbf{v},|u-v|<2, \\ 0, & \text { otherwise }\end{cases}
$$

We wish to prove (11) for $\sigma_{u}$. Equations (81) and (82) yield

$$
\sigma_{n+1 u^{\prime}}\left(x^{\prime}, y^{\prime}\right)=\left(\mathscr{A}_{n} \sigma_{n}\right)_{u^{\prime}}\left(x^{\prime}, y^{\prime}\right)+\tilde{\sigma}_{n u^{\prime}}\left(x^{\prime}, y^{\prime}\right)
$$

with $\tilde{\sigma}_{u^{\prime}}\left(x^{\prime}, y^{\prime}\right)=0$ if $y^{\prime} \in \mathbf{v}^{\prime},\left|u^{\prime}-v^{\prime}\right| \geqq 2$, and

$$
\int_{\mathbf{v}^{\prime}}\left|\tilde{\sigma}_{n u^{\prime}}\left(x^{\prime}, y^{\prime}\right)\right| d y^{\prime} \leqq \delta_{n+1}^{\alpha} e^{-d\left(x^{\prime} \cup v^{\prime} \cup u^{\prime}\right)}
$$

and $\mathscr{A}_{n}$ is the operator

$$
\left(\mathscr{A}_{n} \sigma_{n}\right)_{u^{\prime}}\left(x^{\prime}, y^{\prime}\right)=L^{3} \sum_{u \in L \mathbf{u}^{\prime}} \int d x T_{n}^{L^{2}-1}\left(L x^{\prime}-x\right) \sigma_{n u}\left(x, L y^{\prime}\right) .
$$

Equation (83) is solved by

$$
\sigma_{n}=\mathscr{A}_{n-1} \ldots \mathscr{A}_{0} \tilde{\sigma}_{0}+\mathscr{A}_{n-1} \ldots \mathscr{A}_{1} \tilde{\sigma}_{1}+\ldots+\tilde{\sigma}_{n-1}
$$

with $\tilde{\sigma}_{0}=\sigma_{0}=s_{0}$. We need to estimate

with

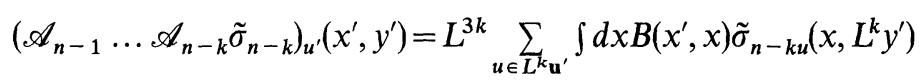

whence

$$
\begin{aligned}
B\left(x^{\prime}, x\right)= & \int d x_{1} \ldots d x_{k-1} T_{n-1}^{L^{2}-1}\left(L x^{\prime}-x_{1}\right) \ldots T_{n-k}^{L^{2}-1}\left(L x_{k-1}-x\right) \\
= & \int d q_{1} \ldots d q_{k} e^{i q_{1} L x^{\prime}-i q_{k} x} \delta\left(q_{1}-L q_{2}\right) \\
& \times \delta\left(q_{2}-L q_{3}\right) \ldots \delta\left(q_{k-1}-L q_{k}\right) \prod_{i} \hat{T}_{n-i}^{L^{2}-1}\left(q_{i}\right) \\
= & L^{-3(k-1)} \int d q e^{i q\left(L x^{\prime}-L^{1-k} x\right)} \prod_{i=1}^{k} \widehat{T}_{n-i}\left(L^{1-i} q\right)^{L^{2}-1},
\end{aligned}
$$

$$
L^{3 k} B\left(x^{\prime}, x\right)=\int d q e^{i q\left(x^{\prime}-L^{-k} x\right)} \prod_{i=1}^{k} \hat{T}_{n-i}\left(L^{-i} q\right)^{L^{2}-1} \leqq C e^{-\frac{3}{2}\left|x^{\prime}-L^{-k} x\right|},
$$

and thus, for $\left|v^{\prime}-u^{\prime}\right|<2$,

$$
\begin{aligned}
\int_{\mathbf{v}^{\prime}} & d y^{\prime}\left|\left(\mathscr{A}_{n-1} \ldots \mathscr{A}_{n-k} \tilde{\sigma}_{n-k}\right)_{u^{\prime}}\left(x^{\prime}, y^{\prime}\right)\right| \\
& \leqq C \delta_{n-k}^{\alpha} L^{-3 k} \sum_{u \in L^{k} \mathbf{u}^{\prime} v \in L^{k \mathbf{v}^{\prime}}} \int d x e^{-\frac{3}{2}\left|x^{\prime}-L^{-k} x\right|} e^{-d(x \cup u \cup v)} \\
& \leqq C \delta_{n-k}^{\alpha} e^{-d\left(x^{\prime} \cup u^{\prime} \cup v^{\prime}\right)}
\end{aligned}
$$

except for $n=k$, where we have $\delta_{n-k}^{\alpha}$ replaced by $\delta_{0}$. Hence, altogether

$$
\int_{\mathbf{v}}\left|\sigma_{n u}(x, y)\right| d y \leqq C\left(\delta_{0}+\sum_{i=1}^{n-1} \delta_{i}^{\alpha}\right) e^{-d(u \cup v \cup x)} \leqq \delta_{0}^{\alpha} e^{-d(u \cup v \cup x)}
$$

and (11) is proven. 
For future reference, we remark that the following analogue of $(11)$ for $s(\xi, y)$ are proven in the same way:

$$
\int_{\mathbf{v}}\left|S_{n u}\left(\xi_{1}, y\right)\right| d y \leqq e^{-d\left(u \cup v \cup u_{1}\right)} A\left(k_{1}\right)^{2 / 3}\left\{\begin{array}{l}
\delta_{n}^{\alpha} \\
\delta_{0}^{\alpha} .
\end{array}\right.
$$

To finish with the deterministic part of the proof, we define the region $R$ of (3.73) as

$$
R_{n+1}=\left\{\mathbf{u} \mid \chi_{u}=0\right\}
$$

Then

Proposition 5. Define $\Delta e$ by

$$
R s+\Delta s=s^{\prime}+\Delta e .
$$

Then

$$
\sum_{Y} \int\left|\Delta e_{Y}(x, y)\right| e^{d(x \cup y \cup Y)} d y \leqq \delta_{0}^{\alpha / 2}
$$

and

$$
\Delta e_{Y}(x, y)=0 ; \text { unless } Y=v, \mathbf{v} \in R .
$$

Proof. We have, see (67),

$$
\Delta e=\sum_{v}(1-E)\left((\mathscr{L} s)_{v}\left(1-\chi_{v}\right)\right) .
$$

The dominant term contributing to (92) is $T^{L^{2}-1} \sigma$ as in (85), yielding the bound. Equation (93) follows from (94) and (90).

We turn now to the stochastic bounds (12) and (15) on $s^{\prime}$, given by (67). The crux of the small field analysis is the study of the linearized RG $\mathscr{L}$ defined in (62) and (63).

Lemma 1. Let s satisfy (12) and (15). Then $\mathscr{L}_{s}$ satisfies the same bounds with $\varepsilon_{n}^{2}$ replaced by $L^{-1}(\log L)^{c} \varepsilon_{n}^{2}$.

Proof. Let us use $\int s_{u}(x, y) d y=0$ to rewrite (63):

$$
\mathscr{L} s=s_{1}+s_{2}+s_{3}
$$

with

$$
\begin{aligned}
s_{1 u^{\prime}}\left(x_{1}^{\prime}, x_{2}^{\prime}\right)= & L^{3} \sum_{u \in L \mathbf{u}^{\prime}} \sum_{t=1}^{L^{2}-2} \int d x_{1} d x_{2} S_{u}\left(x_{1}, x_{2}\right) T^{t}\left(L x_{1}^{\prime}-x_{1}\right) \\
& \times\left(T^{L^{2}-t-1}\left(L x_{2}^{\prime}-x_{2}\right)-T^{L^{2}-t-1}\left(L x_{2}^{\prime}-u\right)\right) \\
s_{2 u^{\prime}}\left(x_{1}^{\prime}, x_{2}^{\prime}\right)= & L^{3} \sum_{u \in L \mathbf{u}^{\prime}} \int d x_{2} s_{u}\left(L x_{1}^{\prime}, x_{2}\right)\left(T^{L^{2}-1}\left(L x_{2}^{\prime}-x_{2}\right)\right. \\
& \left.-T^{L^{2}-1}\left(L x_{2}^{\prime}-u\right)\right) \\
s_{3 u^{\prime}}\left(x_{i}^{\prime}, x_{2}^{\prime}\right)= & L^{3} \sum_{u \in L \mathbf{u}^{\prime}} \int d x_{1} T^{L^{2}-1}\left(L x_{1}^{\prime}-x_{1}\right) s_{u}\left(x_{1}, L x_{2}^{\prime}\right) .
\end{aligned}
$$

Each $s_{i}$ is rewritten, using the formulas (27), (28), and (45). The result is

$$
s_{i u}\left(\xi_{1}^{\prime}, \xi_{2}^{\prime}\right)=\sum_{u \in L \mathbf{u}^{\prime}} \sum_{\xi_{1} \xi_{2}} R_{i}\left(\xi^{\prime}, \xi, u\right) s_{u}\left(\xi_{1}, \xi_{2}\right)
$$


with

$$
\begin{aligned}
R_{1}\left(\xi^{\prime}, \xi, u\right)= & L^{-3} \sum_{t=1}^{L^{2}-2} \int d q_{1} d q_{2} \hat{T}\left(\frac{q_{1}}{L}\right)^{t} \hat{T}\left(\frac{q_{2}}{L}\right)^{L^{2}-t-1} \\
& \times \prod_{i=1}^{2} K\left(k_{i}^{\prime}-q_{i}\right) e^{-i q_{i}\left(v_{i}^{\prime}-\frac{v_{i}}{L}\right)} \\
& \times K\left(k_{1}+\frac{q_{1}}{L}\right)\left(K\left(k_{2}+\frac{q_{2}}{L}\right)-\delta_{k_{2} 0} e^{i \frac{q_{2}}{L}\left(u-v_{2}\right)}\right), \\
R_{2}\left(\xi^{\prime}, \xi, u\right)= & L^{-3} \chi\left(v_{1} \in L \mathbf{v}_{1}^{\prime}\right) \delta_{\frac{k_{1}^{\prime}}{L} k_{2}} e^{i k_{1}\left(v_{1}-L v_{1}^{\prime}\right)} \\
& \times \int d q \hat{T}\left(\frac{q}{L}\right)^{L^{2}-1} K\left(k_{2}^{\prime}-q\right) e^{-i q\left(v_{2}^{\prime}-\frac{v_{2}}{L}\right)} \\
& \times\left(K\left(k_{2}+\frac{q_{2}}{L}\right)-\delta_{k_{2} 0} e^{i \frac{q_{2}}{L}\left(u-u_{2}\right)}\right), \\
R_{3}\left(\xi^{\prime}, \xi, u\right)= & L^{-3} \chi\left(v_{2} \in L v_{2}^{\prime}\right) \delta \frac{k_{2}^{\prime}}{L} k_{2} e^{i k_{2}\left(v_{2}-L v_{2}^{\prime}\right)} \\
& \times \int d q \hat{T}\left(\frac{q}{L}\right)^{L^{2}-1} K\left(k_{1}^{\prime}-q\right) e^{-i q\left(v_{1}^{\prime}-\frac{v_{1}}{L}\right)} K\left(k_{1}+\frac{q}{L}\right) .
\end{aligned}
$$

To bound $R_{1}$, we note that the integrand is analytic in $|\operatorname{Im} q|<3$. In that region (37) holds and furthermore we have

$$
\left|K\left(k_{2}+\frac{q_{2}}{L}\right)-\delta_{k_{2} 0} e^{i \frac{q_{2}}{L}\left(u-v_{2}\right)}\right| \leqq\left|K\left(k_{2}+\frac{q_{2}}{L}\right)-\delta_{k_{2} 0}\right|+C L^{-1}\left|q_{2 L}\right| e^{\frac{1}{2}\left|u-v_{2}\right|} \delta_{k_{2} 0},
$$

where we put

$$
\left(q_{L}\right)_{\mu}= \begin{cases}q_{\mu}, & \left|q_{\mu}\right|<L \\ \pm L, & \left|q_{\mu}\right| \geqq L\end{cases}
$$

Since, from (30),

$$
K(k)=\delta_{k 0}, \quad k \in 2 \pi \mathbf{Z}^{d}, \quad\left|k^{\mu}\right|<2 \pi L^{n},
$$

we get

$$
\left|K\left(k_{2}+\frac{q_{2}}{L}\right)-\delta_{k_{2} 0}\right|=\left|K\left(k_{2}+\frac{q_{2}}{L}\right)-K\left(k_{2}\right)\right| \leqq C L^{-1}\left|q_{2 L}\right|\left(A\left(k_{2}+\frac{q_{2}}{L}\right)+\delta_{k_{2} 0}\right)
$$

which combines with (103), to

$$
(103) \leqq C L^{-1}\left|q_{2 L}\right| e^{\frac{1}{2}\left|u-v_{2}^{\prime}\right|}\left(\delta_{k_{2} 0}+A\left(k_{2}+\frac{q_{2}}{L}\right)\right) .
$$

The sum over $t$ is bounded as [see (39)]

$$
\left|\sum_{t=1}^{L^{2}-2} \hat{T}\left(\frac{q_{1}}{L}\right)^{t} \hat{T}\left(\frac{q_{2}}{L}\right)^{L^{2}-t-1}\right| \leqq \frac{1-a\left(\frac{q_{1}}{L}\right)^{\frac{1}{2} L^{2}}}{1-a\left(\frac{q_{1}}{L}\right)} a\left(\frac{q_{1}}{L}\right) a\left(\frac{q_{2}}{L}\right)^{\frac{1}{2} L^{2}}+(1 \leftrightarrow 2)
$$


and, combining with (107), we get

$$
\frac{1-a\left(\frac{q}{L}\right)^{\frac{1}{2} L^{2}}}{1-a\left(\frac{q}{L}\right)}\left|q_{L}\right|^{n} \leqq \frac{C L^{2}\left|q_{L}\right|^{n}}{1+\left|q_{L}\right|^{2}} \leqq C L^{2} A\left(q_{L}\right)^{\frac{2-n}{3}}
$$

for $n=0,1$. Altogether, (107-109) yield, after shifting the $q$-contour as before,

where

$$
\left|R_{i}\left(\xi^{\prime}, \xi, u\right)\right| \leqq c L^{-2} \prod_{i} e^{-2\left|v_{i}^{\prime}-L^{-1} v_{i}\right|} e^{\frac{1}{2}\left|u-v_{2}\right|} r_{i}\left(k^{\prime}, k\right),
$$

$$
\begin{gathered}
r_{1}\left(k^{\prime}, k\right)=\int d q_{1} d q_{2} \prod A\left(k_{1}^{\prime}-q_{i}\right) A\left(k_{1}+\frac{q_{1}}{L}\right)\left(\delta_{k_{2} 0}+A\left(k_{2}+\frac{q_{2}}{L}\right)\right) \\
\times\left(A\left(q_{1 L}\right)^{2 / 3} a\left(\frac{q_{1}}{L}\right) a\left(\frac{q_{2}}{L}\right)^{\frac{L^{2}}{2}}\right)\left|q_{2 L}\right|+\left(A\left(q_{2 L}\right)^{1 / 3} a\left(\frac{q_{1}}{L}\right)^{\frac{L^{2}}{2}} a\left(\frac{q_{2}}{L}\right)\right) \\
r_{2}\left(k^{\prime}, k\right)=L^{-2} \frac{\delta k_{1}^{\prime} k_{1}}{L} \int d q a\left(\frac{q}{L}\right)^{L^{2}-1} A\left(k_{2}^{\prime}-q\right)\left(\delta_{k_{2} 0}+A\left(k_{2}+\frac{q}{L}\right)\left|q_{L}\right|\right) \\
r_{3}\left(k^{\prime}, k\right)=L^{-1} \frac{\delta k_{2}^{\prime}}{L} k_{2} \int d q a\left(\frac{q}{L}\right)^{L^{2}-1} A\left(k_{1}^{\prime}-q\right) A\left(k_{1}+\frac{q}{L}\right)
\end{gathered}
$$

Now we use Hölder's inequality in the following form

Lemma 2. Let $\{\gamma(x)\}_{x \in \mathbf{Z}^{d}}$ be random variables with

$$
E \exp (t \gamma(x)) \leqq \exp \left(t^{2} \mathscr{G}(x)\right)
$$

then

$$
E \exp \left(t \sum_{x} \gamma(x)\right) \leqq \exp \left(c t^{2} \sum_{x} e^{a|x|}|\mathscr{G}(x)|\right)
$$

for any $a>0, c=c(a)$.

Proof. Let first $\left\{\sigma_{i}\right\}_{i=1}^{n}$ be random variables with $E e^{t \sigma_{i}} \leqq e^{t^{2} g_{i}}$. By Hölder,

$$
\begin{gathered}
E e^{t \Sigma \sigma_{i}} \leqq\left(\left(E e^{p t \sigma_{1}}\right)^{p^{-1}}\right)\left(\left(E e^{q t \Sigma \sigma_{i}}\right)^{q-1}\right) \\
\leqq\left(\left(E e^{p t \sigma_{1}}\right)^{p^{-1}}\right)\left(\left(E e^{p q t \sigma_{2}}\right)^{(p q)^{-1}}\right)\left(\left(E e^{q^{2} t \Sigma_{3} \sigma_{\imath}}\right)^{q-2}\right) \ldots \leqq e^{p t^{2}{ }_{i=1}^{n} q^{i-1} g_{i}} .
\end{gathered}
$$

Apply this first to

$$
\beta(r)=\sum_{|x| \in[r, r+1)} \gamma(x)
$$

to get (take $p=r^{d-1}$ so $q^{n} \leqq C$ )

$$
E e^{t \beta(r)} \leqq \exp \left(C t^{2} r^{d-1} \sum_{|x| \in[r, r+1)}|\mathscr{G}(x)|\right)
$$


Next apply (115) to

$$
\sum_{x \in \mathbf{Z}^{3}} \gamma(x)=\sum_{r=0}^{\infty} \beta(r)
$$

getting

$$
E \exp \left(t \sum_{x} \gamma(x)\right) \leqq \exp \left(C p t^{2} \sum_{r=0}^{\infty} r^{d-1} q^{r} \sum_{|x| \in[r, r+1)}|\mathscr{G}(x)|\right) .
$$

Taking in (119) $q=e^{\frac{1}{2} a}$, say, (114) follows.

Let now $R=R_{1}+R_{2}+R_{3}$. We want to estimate

$$
E \exp \left(\sum_{k_{1}^{\prime} k_{2}^{\prime}}(\mathscr{L} \phi)_{u^{\prime}}\left(\xi_{1}^{\prime}, \xi_{2}^{\prime}\right) f\left(k_{1}^{\prime}, k_{2}^{\prime}\right)\right)=E \exp \left(\sum_{u \subset L \mathbf{u}^{\prime}} \sum_{v_{1} v_{2}} \sigma_{u}\left(v_{1}, v_{2}\right)\right)
$$

with

$$
\sigma_{u}\left(v_{1}, v_{2}\right)=\sum_{k_{i}^{\prime} k_{i}} f\left(k_{1}^{\prime}, k_{2}^{\prime}\right) R\left(\xi^{\prime}, \xi, u\right) \phi_{u}\left(\xi_{1}, \xi_{2}\right)
$$

By induction

$$
E\left(e^{\sigma_{u}\left(v_{1}, v_{2}\right)}\right) \leqq \exp \varepsilon^{2}\left[\sum_{k_{i}^{\prime} k_{i}}\left|f\left(k_{1}^{\prime}, k_{2}^{\prime}\right) \| R\left(\xi^{\prime}, \xi, u\right)\right| C_{u}\left(\xi_{1}, \xi_{2}\right)\right]^{2} .
$$

Use now (110)-(113) and (16) to estimate the $[-]$ in (122):

$$
\begin{aligned}
{[-] \leqq } & C L^{-2} e^{-2 d\left(u \cup v_{1} \cup v_{2}\right)} \prod_{i} e^{-2\left|v_{t}^{\prime}-L^{-1} v_{\imath}\right|} e^{\frac{1}{2}\left|u-v_{2}\right|} \\
& \times \sum_{k_{i}^{\prime} k_{i}}\left|f\left(k_{1}^{\prime}, k_{2}^{\prime}\right)\right| r\left(k^{\prime}, k\right) A\left(k_{1}\right)^{2 / 3} A\left(k_{2}\right)^{1 / 3} .
\end{aligned}
$$

Now, the $k_{i}$-sums are bounded, and we get:

$$
\begin{aligned}
\sum_{k_{1} k_{2}} r_{1} A^{2 / 3} A^{1 / 3} \leqq & C \int d q_{1} d q_{2} \prod A\left(k_{i}^{\prime}-q_{i}\right) \\
& \times\left(A\left(\frac{q_{1}}{L}\right)^{\alpha} \dot{A}\left(q_{1}\right)^{2 / 3} A\left(q_{2}\right)^{\frac{2}{3}+\alpha}+A\left(\frac{q_{2}}{L}\right)^{\alpha} A\left(q_{2}\right)^{1 / 3} A\left(q_{1}\right)^{1+\alpha}\right),
\end{aligned}
$$

where we also used:

$$
\begin{gathered}
a\left(\frac{q}{L}\right)^{\frac{L^{2}}{2}} \leqq C A(q)^{1+\alpha} \\
|q| \leqq C A(q)^{-1 / 3}
\end{gathered}
$$

and

$$
A\left(\frac{q}{L}\right) A\left(q_{L}\right) \leqq A(q)
$$

to get

$$
A\left(q_{L}\right)^{p} a\left(\frac{q}{L}\right) \leqq A(q)^{p} A\left(\frac{q}{L}\right)^{\alpha}
$$

for $p=1 / 3$ or $2 / 3$. 
The $q$-integrals are controlled by

$$
\int d q A\left(k^{\prime}-q\right) A(q)^{t} A\left(\frac{q}{L}\right)^{\alpha} \leqq(\log L)^{c} A\left(k^{\prime}\right)^{t}, \quad t<1 .
$$

Thus, finally

$$
(124) \leqq(\log L)^{c} A\left(k_{1}^{\prime}\right)^{2 / 3} A\left(k_{2}^{\prime}\right)^{1 / 3}
$$

For the $r_{2}$ term,

$$
\sum_{k} r_{2} A^{2 / 3} A^{1 / 3} \leqq C L^{-2} A\left(\frac{k_{1}^{\prime}}{L}\right)^{2 / 3} \int d q A(q)^{1+\alpha} A\left(k_{2}^{\prime}-q\right) \leqq C A\left(k_{1}^{\prime}\right)^{2 / 3} A\left(k_{2}^{\prime}\right)^{1 / 3}
$$

and for $r_{3}$,

$$
\sum_{k} r_{3} A^{2 / 3} A^{1 / 3} \leqq C L^{-1} A\left(\frac{k_{2}^{\prime}}{L}\right)^{1 / 3} A\left(k_{1}^{\prime}\right)^{2 / 3} \leqq C A\left(k_{1}^{\prime}\right)^{2 / 3} A\left(k_{2}^{\prime}\right)^{1 / 3} .
$$

Here, we see the scaling that motivates the powers of $A(k)$ : we gain $L^{-1}$ or $L^{-2}$, which dictates the allowed power of $A$. Thus, (122) is bounded by

$$
E \exp \sigma_{u}\left(v_{1}, v_{2}\right) \leqq \exp B(f) D_{u}\left(v^{\prime}, v\right)
$$

with

$$
D_{u}\left(v^{\prime}, v\right)=e^{-2 d\left(u \cup v_{1} \cup v_{2}\right)} \prod_{i} e^{-4\left|v_{\imath}^{\prime}-L^{-1} v_{\imath}\right|}
$$

and

$$
B(f)=\varepsilon^{2}(\log L)^{c} L^{-4}\left[\sum_{k_{1}^{\prime} k_{2}^{\prime}} A\left(k_{1}^{\prime}\right)^{2 / 3} A\left(k_{2}^{\prime}\right)^{1 / 3}\left|f\left(k_{1}^{\prime}, k_{2}^{\prime}\right)\right|\right]^{2} .
$$

We now apply Lemma 2, first to get

$$
E \exp \left(\sum_{v_{1}} \sigma_{u}\left(v_{1}, v_{2}\right)\right) \leqq \exp \left[C B(f) \sum_{v_{1}} D_{u}\left(v^{\prime}, v\right) e^{\frac{1}{2}\left|v_{1}-u\right|}\right]
$$

[we took $\left.\gamma(x)=\sigma_{u}\left(x-u, v_{2}\right)\right]$, and then

$$
\begin{aligned}
E \exp \left(\sum_{v_{1} v_{2}} \sigma_{u}\left(v_{1}, v_{2}\right)\right) & \leqq \exp \left[C B(f) \sum_{v_{1} v_{2}} D_{u}\left(v^{\prime}, v\right) \prod_{i} e^{\frac{1}{2}\left|v_{i}-u\right|}\right] \\
& \leqq \exp \left[C B(f) e^{-4 d\left(u^{\prime} \cup v_{1}^{\prime} \cup v_{2}^{\prime}\right)}\right] \equiv \exp g
\end{aligned}
$$

where

$$
\begin{gathered}
d\left(u \cup v_{1} \cup v_{2}\right)+\sum_{i=1}^{2} 4\left|v_{1}^{\prime}-L^{-1} v_{i}\right| \geqq \frac{4}{L} d\left(u \cup L v_{1}^{\prime} \cup L v_{2}^{\prime}\right) \\
\geqq \frac{4}{L} d\left(L u^{\prime} \cup L v_{1}^{\prime} \cup L v_{2}^{\prime}\right)-C=4 d\left(u^{\prime} \cup v_{1}^{\prime} \cup v_{2}^{\prime}\right)-C
\end{gathered}
$$

was used.

Finally, coming to (120), note that $\sigma_{u_{1}}$ and $\sigma_{u_{2}}$ are independent, if $\left|u_{1}-u_{2}\right| \geqq 2$. Thus, divide $L \mathbf{u}^{\prime}$ into $\mathcal{O}(1)$ subsets $W_{i}$ such that $\sigma^{\prime} s$ within a fixed $W_{i}$ are independent. Letting

$$
\sigma_{u}=\sum_{v_{1} v_{2}} \sigma_{u}\left(v_{1}, v_{2}\right)
$$


we get, using Schwartz' inequality a few times, and $\left|W_{i}\right| \sim L^{3}$,

$$
\begin{aligned}
& E \exp ((f, \mathscr{L} \phi))=E \exp \left(\sum_{u \in L \mathbf{u}^{\prime}} \sigma_{u}\right) \leqq \prod_{i}\left(E e^{c \underset{u \in W_{i}}{\sum_{i}} \sigma_{u}}\right)^{1 / c} \\
& \leqq \exp \left((\log L)^{c} L^{-1} \varepsilon^{2}\left[\sum\left|f\left(k_{1}^{\prime} k_{2}^{\prime}\right)\right| C_{u^{\prime}}\left(\xi_{1}^{\prime} \xi_{2}^{\prime}\right)\right]^{2}\right.
\end{aligned}
$$

which proves Lemma 1 for (15).

The proof of (12) is very similar. Let us indicate the main differences. We start with the splitting (95), and write

with

$$
\phi_{i u^{\prime}}^{\prime}\left(x^{\prime}, \xi^{\prime}\right)=\sum_{u} \int d x \sum_{\xi} R_{i}\left(x^{\prime}, \xi^{\prime}, x, \xi, u\right) \phi_{i u}(x, \xi)
$$

$$
\begin{gathered}
R_{1}=\sum_{t=1}^{L^{2}-2} T^{t}\left(L x^{\prime}-x\right) \int d q K\left(k^{\prime}-q\right) \hat{T}\left(\frac{q}{L}\right)^{L^{2}-t-1} \\
\times e^{-i q\left(v^{\prime}-\frac{v}{L}\right)}\left(K\left(k+\frac{q}{L}\right)-\delta_{k 0} e^{-i \frac{q}{L}(u-v)}\right), \\
R_{2}=\delta\left(L x^{\prime}-x\right) \int d q K\left(k^{\prime}-q\right) \hat{T}\left(\frac{q}{L}\right)^{L^{2}-1} \\
\times e^{-i q\left(v^{\prime}-\frac{v}{L}\right.}\left(K\left(k+\frac{q}{L}\right)-\delta_{k 0} e^{-i \frac{q}{L}(u-v)}\right), \\
R_{3}=T^{L^{2}-1}\left(L x^{\prime}-x\right) \int d q \hat{T}\left(\frac{q}{L}\right)^{L^{2}-1} e^{-i q\left(v^{\prime}-\frac{v}{L}\right)} K\left(k+\frac{q}{L}\right) K\left(k^{\prime}-q\right) .
\end{gathered}
$$

We need an estimate for $T$ :

Thus

$$
T^{t}(x)=t^{-3 / 2} \int e^{i t^{-1 / 2 k x}} \widehat{T}\left(t^{-1 / 2} k\right)^{t} d k \leqq t^{-3 / 2} \exp \left[-2|x| / t^{1 / 2}\right] .
$$

$$
\sum_{t=1}^{\frac{1}{2} L^{2}} T^{t}(x) \leqq[1+|x|]^{-1} \exp \left[-2 L^{-1}|x|\right],
$$

and so, using (110),

$$
\begin{gathered}
\left|R_{1}\right| \leqq C e^{-2\left|x^{\prime}-\frac{x}{L}\right|-2\left|v^{\prime}-\frac{v}{L}\right|+\frac{1}{2}|u-v|}\left[L^{-1}\left[1+\left|L x^{\prime}-x\right|\right]^{-1} r_{1}\left(k, k^{\prime}\right)+L^{-2} \varrho_{1}\left(k, k^{\prime}\right)\right] \\
r_{1}=\int d q A\left(k^{\prime}-q\right) A(q)^{\alpha+2 / 3}\left(\delta_{k 0}+A\left(k+\frac{q}{L}\right)\right) \\
\varrho_{1}=\int d q A\left(k^{\prime}-q\right) A\left(q_{L}\right)^{1 / 3} A\left(\frac{q}{L}\right)^{1+\alpha}\left(\delta_{k 0}+A\left(k+\frac{q}{L}\right)\right) \\
R_{2}=\delta\left(L x^{\prime}-x\right) R_{2}\left(\xi^{\prime}, \xi, u\right) \\
\left|R_{2}\left(\xi^{\prime}, \xi, u\right)\right| \leqq C L^{-1} e^{-2\left|v^{\prime}-L^{-1} v\right|+\frac{1}{2}|u-v|} r_{1}\left(k^{\prime}, k\right) \\
\left|R_{3}\right| \leqq C L^{-3} e^{-2\left|x^{\prime}-\frac{x}{L}\right|-2\left|v^{\prime}-\frac{v}{L}\right|} r_{3}\left(k^{\prime}, k\right) \\
r_{3}=\int d q A\left(k^{\prime}-q\right) A\left(k+\frac{q}{L}\right) A(q)^{1+\alpha}
\end{gathered}
$$


To estimate (12) for $\mathscr{L} \phi$, put

$$
\begin{gathered}
\sigma_{u}(x, v)=\sum_{i=1,3} \sum_{k^{\prime}, k} f\left(k^{\prime}\right) R_{i}\left(x^{\prime}, \xi^{\prime}, x, \xi, u\right) \phi_{u}(x, \xi), \\
\sigma_{u}(v)=\sum_{k^{\prime}, k} f\left(k^{\prime}\right) R_{2}\left(\xi^{\prime}, \xi, u\right) \phi_{u}\left(L x^{\prime}, \xi\right),
\end{gathered}
$$

which allows us to express

$$
E \exp \sum_{k^{\prime}} f\left(k^{\prime}\right)(\mathscr{L} \phi)_{u^{\prime}}\left(x^{\prime}, \xi^{\prime}\right)=E \exp \sum_{v, u \in L \mathbf{u}^{\prime}}\left(\int d x \sigma_{u}(x, v)+\sigma_{u}(v)\right) .
$$

Also, by induction, we know, that

$$
\begin{aligned}
E \exp \left(t \sigma_{u}(x, v)\right) & \leqq \exp \varepsilon^{2} t^{2}\left(\sum_{k^{\prime}, k} \sum_{i=1,3}\left|f\left(k^{\prime}\right) R_{i}\left(x^{\prime}, \xi^{\prime}, x, \xi, u\right)\right| C_{u}(x, \xi)\right)^{2} \\
& \equiv \exp ^{2} G_{u}(x, v), \\
E \exp t \sigma_{u}(v) \leqq & \exp \varepsilon^{2} t^{2}\left(\sum_{k^{\prime}, k}\left|f\left(k^{\prime}\right) R_{2}\left(\xi^{\prime}, \xi, u\right)\right| C_{u}(x, \xi)\right)^{2} \equiv \exp t^{2} G_{u}(v) .
\end{aligned}
$$

The main difference with respect to the analysis of (15) now lies in the $\int d x$ in (148). We write

$$
\int d x \sigma_{u}(x, v)=\sum_{w \in \mathbf{Z}^{d}} \int_{\mathbf{w}} \sigma_{u}(x, v) d x \equiv \sum_{w} \sigma_{u}(w, v),
$$

and apply repeatedly Schwartz' inequality to get (put $L=2^{m}$, some $m$ ):

$$
\begin{aligned}
E \exp t \sigma_{u}(w, v)=E & \exp t L^{-n d} \sum_{x} \sigma_{u}(x, v) \\
& \leqq \prod_{x}\left(E \exp t \sigma_{u}(x, v)\right)^{L^{-n d}} \leqq \exp t^{2} \int_{\mathbf{w}} G_{u}(x, v) .
\end{aligned}
$$

Then, using Lemma 2 and Schwartz' inequality, we get

$$
\begin{aligned}
& E \exp \sum_{v}\left(\int d x \sigma_{u}(x, v)+\sigma_{u}(v)\right) \\
& \quad \leqq \exp \left[c \sum_{v, w} e^{\frac{1}{2}|u-v|+\frac{1}{2}|u-w|} \int_{w} G_{u}(x, v) d x+c \sum_{v} e^{\frac{1}{2}|u-v|} G_{u}(v)\right] .
\end{aligned}
$$

The analysis of the $q$-integrals and $k$-sums is identical to what we did for (15). Collecting terms we find (we shift the contour slightly more)

$$
\begin{aligned}
G_{u}(x, v) \leqq & C e^{-5\left|x^{\prime}-\frac{x}{L}\right|-5\left|v^{\prime}-\frac{v}{L}\right|+|u-v|-4 d(x \cup u \cup v)}\left[\sum_{k^{\prime}} A\left(k^{\prime}\right)^{1 / 3}\left|f\left(k^{\prime}\right)\right|\right]^{2} \\
& \times\left(L^{-1}\left[1+\left|L x^{\prime}-x\right|\right]^{-1}+L^{-2}(\log L)^{c}+L^{-d}\right)^{-2} .
\end{aligned}
$$

Thus,

$$
\begin{aligned}
& \sum_{u \in L \mathbf{u}^{\prime}} \sum_{v, w} e^{\frac{1}{2}|u-v|+\frac{1}{2}|u-w|} \int_{\mathbf{w}} G_{u}(x, v) d x \leqq e^{-4 d\left(u^{\prime} \cup x^{\prime} \cup v^{\prime}\right)} \int d x e^{-\left|x^{\prime}-\frac{x}{L}\right|} \\
& \quad \times\left[L^{-1}\left(1+\left|L x^{\prime}-x\right|\right)^{-1}+L^{-2}(\log L)^{c}+L^{-3}\right]^{2} .
\end{aligned}
$$

The leading term in (155) is

$$
L^{-2} \int d x e^{-\left|x^{\prime}-\frac{x}{L}\right|}\left[1+\left|L x^{\prime}-x\right|\right]^{-2}=L^{-1} \int d x e^{-\left|x^{\prime}-x\right|}\left[L^{-1}+\left|x^{\prime}-x\right|\right]^{-2} \leqq C L^{-1}
$$


We get then

$$
\prod_{u \in L \mathbf{u}^{\prime}}(153) \leqq \exp \left[\varepsilon^{2}(\log L)^{c} L^{-1}\left(\sum_{k^{\prime}}\left|f\left(k^{\prime}\right)\right| C_{u^{\prime}}\left(x^{\prime}, \xi^{\prime}\right)\right)^{2},\right.
$$

yielding (12) from (153) upon using independence in subsets as before. Lemma 1 is proven.

Now we are ready to prove (12) and (15) for $s^{\prime}$. First, we have

Lemma 3. Let $\varrho$ be given by (65). Then the conclusion of Lemma 1 holds for Re $\varrho$ and $\operatorname{Im} \varrho$.

Proof. The only difference with respect to $\mathscr{L} s$ in $\varrho$ is the $\chi_{v^{\prime}}$. Denote $\operatorname{Re} \varrho$ or $\operatorname{Im} \varrho$ by $\varrho$ again, and the exponents in (12) or (15) by $(f, \phi)$. Put $\omega=(f, \mathscr{L} \phi)$. Lemma 1 is summarized by

$$
E e^{t \omega} \leqq e^{t^{2} G(f)^{2}}
$$

Then, using Holder's inequality $E(\omega \chi)^{p} \leqq E\left(|\omega \chi|^{p}\right)$, we get

$$
E e^{t(f, \varrho)}=E \exp t(\omega \chi-E(\omega \chi)) \leqq 1+\sum_{n=2}^{\infty} \frac{(2 t)^{n}}{n !} \max _{m \leqq n} E\left(|\omega \chi|^{m}\right) E\left(|\omega \chi|^{n-m}\right) .
$$

Equation (158) implies

$$
\operatorname{Prob}(|\omega| \in[n G(f),(n+1) G(f)]) \leqq 2 \exp \left[-\frac{1}{4} n^{2}\right]
$$

whereby,

$$
E\left(|\omega \chi|^{m}\right) \leqq G(f)^{m}+2 \sum_{n=1}^{\infty}(n G(f))^{m} \exp \left[-\frac{1}{4} n^{2}\right] \leqq(m !)^{1 / 2}(C G(f))^{m},
$$

and thus

$$
E e^{t(f, \varrho)} \leqq 1+\sum_{n=2}^{\infty}(n !)^{-1 / 2}(C t G(f))^{n} \leqq e^{c t^{2} G(f)^{2}},
$$

yielding the claim.

Proposition 6. Equations (12) and (15) hold for $s^{\prime}$.

Proof. We use Schwartz' inequality and the notation of Lemma 3,

$$
E e^{\left(f, \phi_{u^{\prime}}^{\prime}\right)} \leqq\left(E e^{2(f, \varrho)}\right)^{1 / 2}\left(E e^{2(f, r)}\right)^{1 / 2}
$$

with

$$
r=R s-\mathscr{L} s+\Delta s .
$$

In the bounds (68) and (78) (consider e.g. $i=2$ ) we could have obtained on the lefthand side the exponential factor $\exp (2 d(\ldots))$ in the norm (13). Hence

$$
|(f, r)| \leqq C(L) \delta_{n}^{3 / 2} \sum_{k_{i}}\left|f\left(k_{1}, k_{2}\right)\right| C\left(\xi_{1}, \xi_{2}\right),
$$

and

$$
E(f, r)=0
$$


Let $f$ be such that the right-hand side of $(164)$ is $\leqq 1$. Then

$$
\begin{aligned}
E e^{(f, r)} & =1+\sum_{n=2}^{\infty}(n !)^{-1} E(f, r)^{n} \leqq 1+C E(f, r)^{2} \\
& \leqq \exp \left[C(L) \delta^{3}\left(\sum f\left(k_{1}, k_{2}\right) C\left(\xi_{1}, \xi_{2}\right)\right)^{2}\right] .
\end{aligned}
$$

For $f$ such that the right-hand side of $(164) \equiv x>1$, we have $x^{2}>x$ and (165) holds again. Equation (163), (165) and Lemma 3 yield the claim.

\section{Traps-Upper Bounds}

In this section, we state and prove the upper bounds for the variables $e_{n}$ and $l_{n}$ describing the effective transition probabilities affected by the traps on previous scales. Thus, given an environment $b: \mathbf{Z}^{3} \times \mathbf{Z}^{3} \rightarrow \mathbf{R}$, we will inductively define the trapping region $D_{n}$, at scale $L^{\tilde{n}}$, which will be a union of $L^{-1}$ cubes, and the effective environment

$$
b_{n}=s_{n}+e_{n}+l_{n}: L^{-\tilde{n}} \mathbf{Z}^{3} \times L^{-\tilde{n}} \mathbf{Z}^{3} \rightarrow \mathbf{R} .
$$

The traps are described by the trap density variables

$$
N_{n}: \mathbf{Z}^{3} \rightarrow(1-2 \alpha) \mathbf{Z} \cup\{-\infty\},
$$

and the connection to $D_{n}$ is by

$$
\mathbf{u} \cap D_{n} \neq \phi \Rightarrow \exists v, \quad|u-v|<2, \quad N_{n v} \neq-\infty .
$$

$\left[-\infty\right.$ is just a convenient way to signal when $l_{n}, e_{n}$ become small enough to be absorbed in the small fields, see below. $N_{n}$ are random variables that take the value $-\infty$ with large probability, see Proposition 1 in Sect.9. To define $D$ inductively (we drop $n$, put $D_{n+1}=D^{\prime}$ and use primes to refer to scale $n+1$ ), we set first

$$
D=D_{\ell} \cup D_{s},
$$

with $D_{\ell}$ being a union of unit cubes and $D_{s}$ a union of $L^{-1}$-cubes and, for $n=n_{0}$, $D_{\ell}=D ; D_{\ell}$ is the "dense trap" region and we put

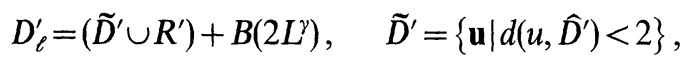

where

$$
\widehat{D}^{\prime}=\left\{\mathbf{v}|| L \mathbf{v} \cap \mathbf{N} \mid \geqq L^{\beta}\right\}
$$

and

$$
N=N_{+} \cup N_{-}=\left\{u \mid N_{u} \geqq 0\right\} \cup\left\{u \mid-\infty<N_{u}<0\right\} .
$$

For $n=n_{0}, N=D ; R^{\prime}$ is given by (5.90). We define

$$
D^{\prime}=D_{\ell}^{\prime} \cup L^{-1}\left(\left(D_{+}+B\left(L^{\alpha}\right)\right) \cup\left(\mathbf{D}_{-} \backslash L \mathscr{D}^{\prime}\right)\right)
$$

and $D_{s}^{\prime}=D^{\prime} \backslash D_{\ell}^{\prime}$. In (6) we have put

$$
D_{+}=\bigcup_{x \in \mathbf{N}_{+}} D(x), \quad D_{-}=D \backslash D_{+}
$$

with $D(x)$ the connected component of $D$ containing $x$. 
Finally, $\mathscr{D}^{\prime}$ is the region of traps, at scale $L^{n+1}$ that are absorbed. To describe $\mathscr{D}^{\prime}$, we need to give the inductive definition of $N$. For $n=n_{0}$, we have defined $N$ in (3.76-3.77).

For the induction, we define variables that signal the nearby presence of "dense traps" in the old and new scales:

$$
n_{u}=\chi\left(L \mathbf{u} \cap\left(L\left(D_{\ell}^{\prime}+B\left(L^{\gamma}\right)\right) \cup\left(D_{\ell}+B\left(L^{\alpha}\right)\right) \neq \phi\right) .\right.
$$

Let us also, for $Y \subset Z^{d}$, set

$$
N(Y)=\min \left(\max _{u \in Y} N(u), 0\right) .
$$

Then, if $N(L \mathbf{u}) \neq \infty$, i.e. if there are traps in $L \mathbf{u}$, we define

$$
\tilde{N}_{u}=N(L \mathbf{u})\left(1-n_{u}\right)+\sum_{v \in L \mathbf{u}}^{+} N_{v}+c_{0} n_{u}-2+2 \alpha \quad \text { if } \quad N(L \mathbf{u}) \neq-\infty
$$

where $\Sigma^{+}$is over positive $N_{v}$ 's. $c_{0}=c_{0}(L)>2-2 \alpha$ is a constant which will be specified in Sect. 8 .

If there are no traps in $L \mathbf{u}$, but nearby there are plenty, we set

$$
\tilde{N}_{u}=c_{0}-2+2 \alpha \quad \text { if } \quad N(L \mathbf{u})=-\infty, \quad n_{u}=1,
$$

and finally

$$
\tilde{N}_{u}=-\infty \text { otherwise }
$$

Now, we can define $\mathscr{D}^{\prime}$ :

$$
\mathscr{D}^{\prime}=\left\{\mathbf{u} \mid \tilde{N}_{u} \leqq-2 n\left(1-2 \alpha+\frac{2 \alpha}{2+\alpha}\right)\right\}
$$

and $N^{\prime}$ :

$$
N_{u}^{\prime}=\left\{\begin{array}{lll}
\tilde{N}_{u} & \text { if } & \tilde{N}_{u}>-2 n\left(1-2 \alpha+\frac{2 \alpha}{2+\alpha}\right) \\
-\infty & \text { if } & \tilde{N}_{u} \leqq-2 n\left(1-2 \alpha+\frac{2 \alpha}{2+\alpha}\right)
\end{array}\right.
$$

Let us try to give some motivation for these complicated, and somewhat arbitrary definitions. $D$ is divided into $D_{\ell}$ and $D_{s}$, and also into $D_{-}$and $D_{+}$. The first division is made according to the size of the traps and the second according to their strength, which is measured by $N$. One sees that, because of the scaling by $L^{-1}$, the size of the $D$ set tends to contract. In $D_{\ell}$, which is very unprobable, we put a large corridor [how unprobably $D_{\ell}$ really is will become clear when we do the probabilistic estimates (see Lemma 9.1b)]. Also, we show below that connected components of $D_{s}$ are made of few $L^{-1}$ cubes (see Lemma 1c, d). We use the corridors to get good Green's function bounds on the probability of hitting a trap. For $D_{\ell}$, we have a large corridor, so that the hitting probability is very small, and a smaller corridor around the part of $D_{+}$which is in $D_{s}$; but here, we use the fact that we deal with few $L^{-1}$ cubes to control the hitting probability. Around $D_{-}$, we do not need a corridor, since there is no $\ell$ there, only $e$ remembering previous traps. If we neglect, for a moment, $n_{u}$ in (10), $N$ tends also to go down, as discussed in Sect. 3, unless several $N^{\prime} s$ fall in the same cube. When $N$ becomes negative, we absorb $\ell$ 
into $e$, and when $N$ becomes as in (13), we absorb $e$ into $s$. Then, that part of $D$ disappears, and $N$ is set to $-\infty$. The role of $n$ is, first, to set $N \neq-\infty$ in the corridors, so that (3) holds, but it is also used to prove the lower bounds on $T+b$, in Sect. 8, which imply the upper bound on $\ell,(28)$ below, which, itself, is needed to reabsorb $\ell$ into $e$. Of course, since $n$ increases $N$ a lot, we must pay a price, in the probabilistic estimates. But, here, we can use the fact that $n_{u} \neq 0$ is very unprobable (see Lemma 9.1, d).

We collect now some facts, proven in the Appendix, implied by our construction of $D$ and $N$.

Lemma 1. a) Equation (3) holds, i.e.

$$
\mathbf{D} \subset\left\{\mathbf{v}|\exists u,| v-u \mid<2, N_{u} \neq-\infty\right\} .
$$

Also,

$$
D_{\ell} \subset D_{+}
$$

b) If $u \notin \tilde{D^{\prime}}$,

c)

$$
\begin{gathered}
|\mathbf{D} \cap L \mathbf{u}| \leqq c L^{\beta} . \\
\forall v,\left|D_{s} \cap \mathbf{v}\right| \leqq L^{-2-2 \alpha} .
\end{gathered}
$$

Moreover, if $u \notin \widetilde{D^{\prime}}$.

$$
\left|D_{s} \cap L \mathbf{u}\right| \leqq L^{-2}
$$

while, if $u \notin \hat{D}^{\prime},\left|D_{s} \cap L \mathbf{u}\right| \leqq L^{-\alpha}$.

d) Let $D(x)$ be the connected component of $D$ containing $x$. If $D(x) \subset D_{s}$,

$$
\begin{gathered}
|D(x)| \leqq L^{-2} \\
\operatorname{diam} D(x)) \leqq 1
\end{gathered}
$$

e) $D_{\ell} \subset D_{+}$, and, if $x \in D_{-} \cup\left(D_{+} \cap L D_{-}^{\prime}\right)$,

$$
D(x) \subset D_{s} .
$$

f) If $x \notin L \widetilde{D^{\prime}}$, then

$$
\left|\left(x+B\left(\frac{L}{2}\right)\right) \cap D\right| \leqq c L^{3} .
$$

The functions $e$ and $\ell$ in (1) have the following properties.

Proposition 1. $e_{Y}$ is a random variable localized in $Y$ and $\ell(x, y)$ is localized in $D(x)$. We have $e_{Y}=\varepsilon_{Y}+E_{Y}$,

$$
\begin{gathered}
\int d y \varepsilon_{Y}(x, y)=\int d y E_{Y}(x, y)=\int d y \ell(x, y)=0, \\
\sum_{Y} \int d y\left|\varepsilon_{Y}(x, y)\right| e^{d(x \cup y \cup Y)} L^{-\frac{N(Y)}{2}} \leqq L^{-\alpha / 2}, \\
N(Y) \neq-\propto \\
\sum_{Y} \int d y\left|E_{Y}(x, y)\right| e^{d\left(x \cup y \cup Y ; Y \cap D_{\ell}\right)} \leqq e^{-L^{\gamma}},
\end{gathered}
$$

and $E_{Y}=0$ if $Y \cap \tilde{D}=\phi ; \varepsilon_{Y}=0$ if $N(Y)=-\infty . \ell(x, y)=0$ if $x \notin D_{+}$,

$$
\int d y|\ell(x, y)| e^{d(y, D(x))} \leqq c,
$$


and, for $x \in \mathbf{u}$

$$
\int_{D_{s} \backslash L \hat{D}} d y|\ell(x, y)| \leqq 1-\beta_{u}+L^{-\frac{\alpha}{3}}
$$

where

$$
\beta_{u}=L^{2-N_{u}} /\left(1+L^{2-N_{u}}\right) .
$$

We will prove (24)-(27) in this section. Equation (28) will be a consequence of the lower bounds for $\ell$ proven in Sect. 8.

Expansion of $b^{\prime}$. Coming back to (1), we now define $e_{n+1}$ and $\ell_{n+1}$ inductively. We have

$$
b^{\prime}\left(x^{\prime}, y^{\prime}\right)=L^{3} \int d \omega_{L x^{\prime} L y^{\prime}}^{L^{2}}[(T+b)(\omega)-E(T+b)(\omega)]
$$

and $s^{\prime}$ is defined by (5.67), (5.69).

Let first $x^{\prime} \notin D_{+}^{\prime}$. Define

$$
\bar{D}=L \tilde{D}^{\prime} \cup\left(L D_{+}^{\prime} \cap D_{+}\right) .
$$

Note that, if $x \notin \bar{D}$, then, by Lemma 1e, $D(x) \subset D_{s}$ and (21) holds. $\bar{D}$ is the region where we cannot expand $b$ in (30). This is because the $D$ region in $L \tilde{D}$ can be very large and then $e$ turns out not to be expandable due to lack of falloff on $D_{\ell}$, see (26), or because $\ell$ in $L D_{+}^{\prime} \cap D_{+}$has no smallness, see (27). One might think that, in $D_{+} \backslash L D_{+}^{\prime}$, there is also a problem. However, it turns out that $\ell$, in that region, is small enough to be expanded, due to $(28,29)$.

We now proceed as in (4.6), only replacing $D$ by $\bar{D}$ :

$$
\begin{aligned}
& \int d \omega(T+b)(\omega)-(T+s)(\omega)=\sum_{k \geqq 2} \sum_{t, t^{\prime}, \underline{v}} \prod_{i=1}^{k-1} \chi\left(\mathbf{v}_{i} \subset \overline{\mathbf{D}}\right) \\
& \quad \times \int d \underline{x} d \underline{y} \chi_{1} \prod_{i=1}^{k}(T+b)_{\bar{D}^{c}}^{t_{i}}\left(x_{i-1}, y_{i}\right) \prod_{i=1}^{k-1}(T+b)_{W\left(y_{i}\right)}^{t_{i}}\left(y_{i}, x_{i}\right)-(b \rightarrow s) .
\end{aligned}
$$

We have the familiar constraints: $x_{0}=L x^{\prime}, y_{k}=L y^{\prime}, \underline{t} \in \mathbf{N}^{k}, \underline{t}^{\prime} \in\left(\mathbf{N}_{+}\right)^{k-1}$, $\sum t_{i}+\sum t_{i}^{\prime}=L^{2}, \underline{v} \in\left(\mathbf{Z}^{d}\right)^{k-1}$, and

$$
\chi_{1}=\prod_{i=1}^{k-1} \chi\left(x_{i} \notin W\left(y_{i}\right)\right) \chi\left(y_{i} \in \mathbf{v}_{i} \cap \bar{D}\right)
$$

with no constraint on $x_{k-1}$ if $t_{k}=0$. $W(y)$, for $y \in \bar{D}$, is given by

$$
W(y)=\begin{array}{ll}
\text { c.c. of } L D^{\prime} & \text { if } \quad y \in L D^{\prime} \\
\text { c.c. of }\left(D_{+}+B\left(L^{\alpha}\right)\right) \cup D & \text { if } \quad y \in \bar{D} \backslash L \tilde{D}^{\prime}
\end{array}
$$

such that $y \in W(y)$.

As before, we localize the various terms in (32). First, write

where

$$
\sum_{v} \chi(\mathbf{v} \subset \overline{\mathbf{D}})=\sum_{b=1,2} \sum_{W, v} \chi\left(W \in E_{b}\right) \chi(\mathbf{v} \subset \overline{\mathbf{D}} \cap \mathbf{W})
$$

$$
E_{1(2)}=\left\{W \mid W=W(y), y \in \bar{D} \backslash L \widetilde{D}^{\prime}\left(y \in L \widetilde{D}^{\prime}\right)\right\},
$$

and the sum over $W$ runs over connected sets in $\mathbf{Z}^{d}$. Then, combine (33) and (35):

$$
\sum_{v} \chi(\mathbf{v} \subset \overline{\mathbf{D}} \cap \mathbf{W}) \int d y \chi(y \in \mathbf{v} \cap \bar{D})=\int d y \chi(y \in \mathbf{W} \cap \bar{D}) .
$$


Next, expand $(T+b)_{\bar{D} c}$ :

$$
(T+b)_{\bar{D}^{c}}^{t}(x, y)=\sum_{k, t} \sum_{Y} \sum_{u, v} \int d \underline{x} d \underline{\underline{u}} \chi_{2} \prod_{i=1}^{k} T_{D^{c}}^{\bar{t}_{c}}\left(x_{i-1}, y_{i}\right) \prod_{i=1}^{k-1} Q_{a_{i} Y_{i}}\left(y_{i}, x_{i}\right),
$$

where $\underline{t} \in \mathbf{N}^{k}, \sum t_{i}=t-k+1, \underline{a} \in\{1,2,3,4\}^{k-1}, \underline{Y}=\left(Y_{1}, \ldots, Y_{k-1}\right)$, and $\underline{u}, \underline{v} \in\left(\mathbf{Z}^{3}\right)^{k-1}$. $Y_{i}$ are unions of unit cubes and it is convenient to use the notation

$$
Q_{1}=\varepsilon, \quad Q_{2}=E, \quad Q_{3}=s
$$

and

$$
Q_{4 Y}(y, x)=\ell(y, x) \chi(Y=D(y))
$$

Finally,

$$
\chi_{2}=\prod^{\prime} \chi\left(x_{i} \in \mathbf{u}_{i} \backslash \bar{D}\right) \chi\left(y_{i} \in \mathbf{v}_{i} \backslash \bar{D}\right) .
$$

In (38), we further expand

$$
T_{\bar{D}^{c}}^{t}(x, y)=\sum_{k, t, t^{\prime}} \sum_{v} \prod_{i=1}^{k-1} \chi\left(\mathbf{v}_{i} \subset \overline{\mathbf{D}}\right) \int d \underline{x} d \underline{y} \chi_{1} \prod_{i=1}^{k} T^{t_{i}}\left(x_{i-1}-y_{i}\right) \prod_{i=1}^{k-1}\left(-T_{W_{i}}^{t_{i}}\left(y_{i}, x_{i}\right)\right)
$$

with $\tilde{W}_{i}=W\left(y_{i}\right) \cap \bar{D}^{c}$.

Now consider $(T+b)_{W}$ in (32). This has terms in $b$ that are not localized near $W$. We still expand them. Let

$$
\chi_{x y}(Y \mid W)=\chi\left(d(x \cup y \cup Y ; W)>L^{\gamma}\right)
$$

and denote it by $\chi(Y)$ when no confusion can arise. Set

$$
\hat{b}_{W}(x, y)=\ell(x, y)+\sum_{i, Y} Q_{i Y}(x, y)(1-\chi(Y))
$$

$[i=1,2,3$, see (39)], whence

$$
b=\widehat{b}_{W}+\sum_{i, Y} Q_{i Y} \chi(Y) .
$$

Lemma 2. Given $W \in E_{1} \cup E_{2}, \hat{b}_{W}(x, y)$ is localized in $W+B\left(L^{y}\right)$, if $x \in W$.

Proof. By construction, $W \supset D(x)$ for all $x \in W$ and $\ell(x, y)$ is localized in $D(x)$, by Proposition 1. The $Q_{i Y}$ 's are localized in $Y$ and, by (43), the $Y$ 's contributing to (44) are in $W+B\left(L^{\gamma}\right)$.

Using (45), we get

$$
\begin{aligned}
(T+b)_{W}^{t}(x, y)= & \sum_{k, t} \sum_{a, Y} \int d \underline{x} d \underline{y} \chi\left(x_{i}, y_{i} \in W\right) \prod_{i=1}^{k}(T+\widehat{b})_{W}^{t_{i}}\left(x_{i-1}, y_{i}\right) \\
& \times \prod_{i=1}^{k-1} Q_{a_{i} Y_{i}}\left(y_{i}, x_{i}\right) \chi\left(Y_{i}\right)
\end{aligned}
$$

with $a_{i}=1,2,3$, and standard constraints.

It is convenient to collect in (46) terms with $\cup Y_{i}=Z$, and write

$$
(50)=\sum_{Z} Q_{5 Z}^{t}(x, y) \text {. }
$$

$Q_{5 Z}^{t}$ is localized in $Z \cup\left(W+B\left(L^{\gamma}\right)\right)$. 
We may now localize (32). Insert (35) and (37), (38) and (42), (46) and (47) into (32):

$$
\begin{aligned}
& L^{3} \int d \omega {[(T+b)(\omega)-(T+s)(\omega)] } \\
&= L^{3} \sum_{k \geqq 2} \sum_{t, t^{\prime}} \sum_{a, b} \sum_{Z} \sum_{u, v, W}^{\prime} \prod_{a_{i}>4} \chi\left(\mathbf{W}_{i} \in E_{b_{i}}\right) \int d \underline{x} d \underline{y} \chi_{3} \prod_{i=1}^{k} T^{t_{i}}\left(x_{i-1}-y_{i}\right) \\
& \quad \times \prod_{i=1}^{k-1} Q_{a_{i} Z_{i}}^{t_{i}}\left(y_{i}, x_{i}\right)-(b \rightarrow s),
\end{aligned}
$$

where the various constraints are: $\underline{t \in \mathbf{N}^{k}}, \underline{t}^{\prime} \in \mathbf{N}_{+}^{k-1}, a_{i}=1, \ldots, 6, Z_{i}$, are unions of unit cubes, $W_{i}$ connected subsets of $\mathbf{Z}^{3}$ and, in $\sum^{\prime}$, we sum over $u_{i}$, $v_{i}$ for $a_{i} \leqq 4, W_{i}$ for $a_{i}>4 . \chi_{3}$ is given by

$$
\chi_{3}=\prod_{a_{i} \leqq 4}^{\prime} \chi\left(x_{i} \in \mathbf{u}_{i} \backslash \bar{D}\right) \chi\left(y_{i} \in \mathbf{v} \backslash \bar{D}\right) \prod_{a_{\imath}>4}^{\prime} \chi\left(y_{i} \in W_{i} \cap \bar{D}\right) \chi\left(x_{i} \notin W_{i}\right) .
$$

$Q_{5}$ is given by (47), and

$$
Q_{6 Z}^{t}(y, x)=-T_{W \cap \bar{D}^{c}}^{t}(y, x) \delta_{Z, \phi} .
$$

In (48), we have $a_{i} \neq 3$ for at least one $i$, because of the subtraction $(b \rightarrow s)$. Equation (48) is the desired expansion. We will now collect terms to define $\varepsilon^{\prime}$ and $E^{\prime}$ (recall, that $x^{\prime} \in D_{+}^{\prime}$ so $\ell^{\prime}=0$ ). We write

$$
(48)-E(48)=\sum_{Y^{\prime}}\left(\tilde{\varepsilon}_{Y^{\prime}}+\widetilde{E}_{Y^{\prime}}\right) \text {, }
$$

where $\tilde{\varepsilon}_{Y^{\prime}}$ collects terms with

$$
Y^{\prime}=\left[L^{-1}\left(\bigcup_{i} \mathbf{u}_{i} \bigcup_{i} \mathbf{v}_{i} \bigcup_{i} \mathbf{W}_{i} \bigcup_{i} Z_{i}\right)\right]
$$

such that, if $a_{i}=2$ or 5 ,

and

$$
Z_{i D} \equiv Z_{i} \cup \bigcup_{u}\left\{L \mathbf{u} \| L \mathbf{u} \cap N \mid>L^{\beta}\right\}=\emptyset
$$

$$
b_{i}=1 \quad \text { if } \quad a_{i}>4 .
$$

$\widetilde{E}_{Y^{\prime}}$ collects terms with (52) such that

$$
\exists i: a_{i}=2 \text { or } 5, \quad Z_{i D} \neq \emptyset \text { or } a_{i}>4, \quad b_{i}=2 .
$$

Finally, we put

$$
\varepsilon_{Y^{\prime}}^{\prime}\left(x^{\prime}, y^{\prime}\right)=\tilde{\varepsilon}_{Y^{\prime}}\left(x^{\prime}, y^{\prime}\right)-T^{\prime}\left(x^{\prime}-y^{\prime}\right) \int d z \tilde{\varepsilon}_{Y^{\prime}}\left(x^{\prime}, z\right)+\Delta \varepsilon_{Y^{\prime}}\left(x^{\prime}, y^{\prime}\right)-\Delta s_{Y^{\prime}}\left(x^{\prime}, y^{\prime}\right),
$$

where [see (5.93), (5.94)],

$$
\Delta \varepsilon_{v^{\prime}}=(1-E)\left((\mathscr{L} s)_{v^{\prime}}\left(1-\chi_{v^{\prime}}\right)\right) ; \quad \Delta \varepsilon_{Y^{\prime}}=0 \quad \text { if } \quad\left|Y^{\prime}\right|>1
$$

and $\Delta s$ is defined in Sect. 7. We define $E^{\prime}$ similarly, without the $\Delta \varepsilon, \Delta s$.

To complete the induction, let now $x^{\prime} \in D_{+}^{\prime}$. We modify (48) slightly: now $t_{1}=0$, $a_{1}=5$, and $W\left(L x^{\prime}\right)=L D^{\prime}\left(x^{\prime}\right)$. Let $\widetilde{\ell}\left(x^{\prime}, y^{\prime}\right)$ collect all the terms with

$$
\cup u_{i} \cup v_{i} \cup W_{i} \cup Z_{i} \subset L\left(D^{\prime}\left(x^{\prime}\right)+B\left(2 L^{\gamma}\right)\right)
$$

and define $\ell^{\prime}$ by

$$
\ell^{\prime}=\ell^{\prime}-T^{\prime} \int d z \tilde{\ell}(\cdot, z)
$$


$\varepsilon^{\prime}$ and $E^{\prime}$ are defined as above, for

$$
Y^{\prime} \not D^{\prime}\left(x^{\prime}\right)+B\left(2 L^{\gamma}\right)
$$

and set to zero otherwise.

The inductive definition of $b^{\prime}$ is completed.

Proof of Proposition 1. We first establish the localization properties of $e^{\prime}$ and $\ell^{\prime}$. Note that, in (48), $Q_{a Z}$ are localized in $Z$ for $a \leqq 4$ and in $Z \cup\left(W+B\left(L^{\gamma}\right)\right)$ for $a>4$ [recall (47) and (50)]. $\chi\left(W_{i} \in E_{b}\right)$ is localized in $W_{i}+B\left(3 L^{1+\gamma}\right)$ : because of the corridors (5), (6), the statement $W \in E_{b}$ is only localized in such a neighbourhood of $W$ (see Lemma 4.1). Similar statement hold for the other $\chi$ 's in (48), (49). Therefore each term in (48) is localized in

$$
\left(\cup \mathbf{u}_{i} \cup \mathbf{v}_{i} \cup \mathbf{W}_{i} \cup Z_{i}\right)+B\left(3 L^{1+\gamma}\right) .
$$

Thus, since $L^{-1}\left(3 L^{1+\gamma}+6 L^{\gamma}\right)<6 L^{\gamma}$ [see (4.19)] $e^{\prime}$ is properly localized. For $\ell^{\prime}$, we use (57) and $L^{-1}\left(3 L^{1+\gamma}+2 L^{1+\gamma}+6 L^{\gamma}\right)<6 L^{\gamma}$.

Also, observe that (1) holds: we have, from $(30,48,50)$,

$$
b^{\prime}=s^{\prime}+\tilde{\varepsilon}+\tilde{E}
$$

for $x^{\prime} \notin D_{+}^{\prime}$. But $\int s^{\prime}=\int b^{\prime}=0$. Hence $\int(\tilde{\varepsilon}+\widetilde{E})=0$ and the subtraction in (55) (and for $\left.E^{\prime}\right)$ may be done without spoiling (1). Next, we see that (24) follows readily, due to the subtraction in (55) and (58), since $\int T^{\prime}(x) d x=1$. Moreover, $\int \Delta \varepsilon=\int \Delta s=0$ (see Sects. 5 and 7). Therefore, $\int \varepsilon_{Y^{\prime}}^{\prime}=\int E_{Y}^{\prime}=0$ as claimed. For $x^{\prime} \in D_{+}^{\prime}$ the argument is similar.

Now, observe that (55), (53), (36), (34), and (52) imply, by construction, $E_{Y^{\prime}} \neq 0$ only when $Y^{\prime} \cap \widetilde{D}^{\prime} \neq \phi$. For $\varepsilon_{Y^{\prime}}$, we shall show in Sect. 7 that, if $N\left(Y^{\prime}\right)=-\infty$,

$$
\tilde{\varepsilon}_{Y^{\prime}}\left(x^{\prime}, y^{\prime}\right)-T^{\prime}\left(x^{\prime}-y^{\prime}\right) \int d z \tilde{\varepsilon}_{Y^{\prime}}\left(x^{\prime}, z\right)=\Delta s_{Y^{\prime}}\left(x^{\prime}, y^{\prime}\right),
$$

and, since $\Delta \varepsilon_{Y^{\prime}} \neq 0$ only when $Y^{\prime}=u \in R, \Delta \varepsilon_{Y^{\prime}} \neq 0$ implies $N\left(Y^{\prime}\right) \neq-\infty$. So, $\varepsilon_{Y^{\prime}}=0$ if $N\left(Y^{\prime}\right)=-\infty$. We turn now to the proof of (25-27); we shall start with some general facts and then divide the proof as follows:
A. Proof of (25) for $x^{\prime} \notin D_{+}^{\prime}$.
B. Proof of (26) for $x^{\prime} \notin D_{+}^{\prime}$.
C. Proof of $(25-27)$ for $x^{\prime} \in D_{+}^{\prime}$.

All our bounds will be proven for $\tilde{\varepsilon}, \tilde{E}, \tilde{\ell}$. It is easy to deduce $(25-27)$ from similar bounds on $\tilde{\varepsilon}, \widetilde{E}, \tilde{\ell}$ and (55), (58) using the exponential decay of $T(x-y)$ and the trivial bound

$$
d_{a}\left(x^{\prime}, y^{\prime}, Y^{\prime}\right) \leqq d_{a}\left(x^{\prime}, z, Y^{\prime}\right)+\left|x^{\prime}-y^{\prime}\right| \quad \text { for } \quad a=1,2,4
$$

where we use notation,

$$
\begin{gathered}
d_{a}(x, y, Y)=d(x \cup y \cup Y), \quad a=1,3, \\
d_{2}(x, y, Y)=d\left(x \cup y \cup Y ; Y \cap D_{\ell}\right), \\
d_{4}(x, y, Y)=d(y, D(x))(Y=D(x) \text { for } a=4), \\
d_{5}(x, y, Y)=\min \left(\sum_{i=1}^{n} d\left(x \cup y \cup Z_{i} ;\left(Z_{i} \cap D_{\ell}\right) \cup W(x)\right),\right.
\end{gathered}
$$


where the minimum is taken over all families $\left(Z_{i}\right)_{i=1}^{n}$ satisfying $\bigcup_{i=1}^{n} Z_{i}=Y$,

$$
d_{6}(x, y, Y)=d(y, W(x))(Y=\emptyset \text { for } a=6) .
$$

In the next lemma, proven in the Appendix, we shall bound the distance factors in (25-27) in terms of the variables appearing in (48).

Lemma 3. a) For each term of (48) contributing to $\tilde{\varepsilon}_{Y^{\prime}}$ i.e. satisfying (52-54), we have

$$
d\left(x^{\prime} \cup y^{\prime} \cup Y^{\prime}\right) \leqq c k+L^{-1}\left(\sum_{i=1}^{k}\left|x_{i-1}-y_{i}\right|+2 \sum_{i=1}^{k-1} d_{a_{i}}\left(y_{i}, x_{i}, Z_{i}\right)\right) \text {. }
$$

b) For each term of (48) contributing to $\widetilde{E}_{Y^{\prime}}$, i.e. satisfying $(52,55)$, we have

$$
d\left(x^{\prime} \cup y^{\prime} \cup Y^{\prime} ; Y^{\prime} \cap D_{\ell}^{\prime}\right) \leqq c k+L^{-1}\left(\sum_{i=1}^{k}\left|x_{i-1}-y_{i}\right|+2 \sum_{i=1}^{k-1} d_{a_{i}}\left(y_{i}, x_{i}, Z_{i}\right)\right) .
$$

c) For each term of (48) contributing to $\widetilde{\ell}\left(x^{\prime}, y^{\prime}\right)$, i.e. satisfying (57), we have

$$
d\left(y^{\prime}, D\left(x^{\prime}\right)\right) \leqq c k+L^{-1}\left(\sum_{i=1}^{k}\left|x_{i-1}-y_{i}\right|+2 \sum_{i=1}^{k-1} d_{a_{1}}\left(y_{i}, x_{i}, Z_{i}\right)\right) \text {. }
$$

It will be useful to have bounds on the integral over $x$ of the $Q_{a z}^{t^{\prime}}(y, x)$ factors in (48). These can be summarized in

\section{Lemma 4.}

$$
\left.\sum_{t} \sum_{Z} \int d x \chi(x) \mid Q_{a Z}^{t}(y, x)\right) \mid e^{\frac{1}{2} d_{a}(y, x, Z)} \leqq \begin{cases}L^{-a / 2}, & a=1 \\ \exp \left(-L^{\gamma}\right), & a=2,3 \\ c, & a \geqq 4,\end{cases}
$$

where $\chi(x)=\chi(x \notin W(Y))$ for $a>4$ and $\chi(x)=1$, for $a \leqq 4$.

For $a \leqq 4(t=0$, then) this lemma follows from (25-27) and (5.11), using $c \delta_{0}^{\alpha} \leqq \exp \left(-L^{\gamma}\right)$. For $a=5,6$, the proof is given in the Appendix.

A. Proof of (25) for $x^{\prime} \notin D_{+}^{\prime}$. The proof of (25) follows from the following estimates.

$$
\begin{gathered}
\sum_{Y} \int d y\left|\tilde{\varepsilon}_{Y}^{\prime}(x, y)\right| e^{d(x \cup y \cup Y)} \leqq \frac{L^{-\alpha / 2}}{2}, \\
\sum_{\substack{Y \\
N(Y) \neq 0,-\infty}} \int d y\left|\tilde{\varepsilon}_{Y}^{\prime}(x, y)\right| e^{d(x \cup y \cup Y)} L^{-\frac{N(Y)}{2}} \leqq \frac{L^{-\alpha / 2}}{2} .
\end{gathered}
$$

1) Proof of (68). Using (48), (51-54) and Lemma 3a, we get

$$
\begin{aligned}
\sum_{Y} \int d y\left|\tilde{\varepsilon}_{Y}^{\prime}(x, y)\right| e^{d(x \cup y \cup Y)} \leqq 2 \sum_{k \geqq 2} e^{c k} \sum_{\underline{t t^{\prime}}} \sum_{a} \sum_{Z} \int d \underline{x} d \underline{y} \chi_{4} \\
\quad \times \prod_{i=1}^{k} T^{t_{\imath}}\left(x_{i-1}-y_{i}\right) e^{L^{-1}\left|x_{i-1}-y_{i}\right|} \\
\quad \times \prod_{i=1}^{k-1}\left|Q_{a_{\imath} Z_{\imath}}^{t_{i}}\left(y_{i}, x_{i}\right)\right| e^{2 L^{-1} d_{a_{\imath}}\left(y_{l}, x_{i}, Z_{\imath}\right)}+|(b \rightarrow s)|,
\end{aligned}
$$

where

$$
\chi_{4}=\prod_{a_{i} \leqq 4}^{\prime} \chi\left(x_{i} \notin \bar{D}\right) \chi\left(y_{i} \notin \bar{D}\right) \prod_{a_{i}>4}^{\prime} \chi\left(y_{i} \in \bar{D} \backslash L \widetilde{D}^{\prime}\right) \chi\left(x_{i} \notin W\left(y_{i}\right)\right) .
$$


Also, here, $d \underline{y}=\prod_{i=1}^{k} d y_{i}$ and we used $L^{d} \int d y f(L y)=\int d y_{k} f\left(y_{k}\right)$. The factor 2 in (70) comes from the subtraction of the expectation value in (51); we shall consider only the first term in $(70)$, the $(b \rightarrow s)$ term being treated similarly. It will be convenient to bound (70) in terms of the following quantities:

$$
\tilde{A}_{1}(x, t)=\sum_{Z} \int d y d z \chi(y \notin \bar{D}) T^{t}(x-y) e^{2 L^{-1}|x-y|}\left|Q_{1 Z}(y, z)\right| e^{\frac{1}{2} d(y \cup z \cup Z)} L^{-N(Z) / 2},
$$

and $A_{1}(x, t)$ is defined similarly without $L^{-N(Z) / 2}$,

$$
A_{a}(x, t)=\sum_{Z} \int d y d z \chi(y \notin \bar{D}) T^{t}(x-y) e^{2 L^{-1}|x-y|}\left|Q_{a Z}(y, z)\right| e^{\frac{1}{2} d_{a}(y, z, Z)},
$$

for $a=2,3,4$,

$$
A_{a}(x, t)=\sum_{Z, t^{\prime}} \int d y d z \chi\left(y \in \bar{D} \backslash L \widetilde{D}^{\prime}\right) \chi(z \notin W(y)) T^{t}(x-y) e^{2 L^{-1}|x-y|}
$$

for $a=5,6$, and

$$
\times\left|Q_{a Z}^{t^{\prime}}(y, z)\right| e^{\frac{1}{4} d_{a}(y, z, Z)}
$$

$$
A_{a}(x)=\sum_{t=0}^{L^{2}} A_{a}(x, t)
$$

for all $a$. For the $A$ 's we have the bounds:

\section{Lemma 5.}

a)

$$
\begin{aligned}
& \tilde{A}_{1}(x, 0) \leqq L^{-\alpha / 2}, \\
& \tilde{A}_{1}(x, t) \leqq L^{-\alpha / 3} t^{-3 / 2}, \quad t \neq 0, \\
& A_{1}(x) \leqq L^{-\alpha / 3}\left(d\left(x, D_{+}\right)+1\right)^{-1}+L^{-\alpha},
\end{aligned}
$$

b)

$$
A_{a}(x) \leqq \exp \left(-L^{\gamma} / 2\right), \quad a=2,3,
$$

c)

$$
\begin{aligned}
& A_{4}(x, 0) \leqq c \chi\left(x \in D_{+} \backslash \bar{D}\right), \\
& A_{4}(x, t) \leqq L^{-\alpha} t^{-3 / 2}, \quad t \neq 0, \\
& A_{4}(x) \leqq c\left(\chi\left(x \in D_{+} \backslash \bar{D}\right)+L^{-\alpha}\right),
\end{aligned}
$$

d)

$$
A_{\alpha}(x) \leqq c L^{\beta}\left(d\left(x, D_{\ell}\right)+1\right)^{-1}+L^{-\alpha}, \quad a=5,6 .
$$

To control the fact that we do not have a small factor in c) and d) above we introduce

$$
B_{a a^{\prime}}(x)=\sum_{Z, t} \int d y d z \chi(y \notin \bar{D}) T^{t}(x-y) e^{2 L^{-1}|x-y|} Q_{a Z}(y, z) \mid e^{L^{-\gamma} d_{a}(y, z, Z)} A_{a^{\prime}}(z)
$$

for $a \leqq 4$ and

$$
\begin{aligned}
B_{a a^{\prime}}(x)= & \sum_{Z} \sum_{t, t^{\prime}} \int d y d x \chi\left(y \in \bar{D} \backslash L \widetilde{D}^{\prime}\right) \chi(z \notin W(y)) T^{t}(x-y) \\
& \times e^{2 L^{-1}|x-y|}\left|Q_{a Z}^{t^{\prime}}(y, z)\right| e^{\frac{1}{4} d_{a}(y, z, Z)} A_{a^{\prime}}(z)
\end{aligned}
$$

for $a=5,6$.

The $B$ 's satisfy better bounds:

\section{Lemma 6.}

a)

$$
B_{a a^{\prime}}(x) \leqq L^{-\alpha / 3} L^{2 \beta} \quad \text { unless } a=5,6 \text { and } a^{\prime}=4 \text {, }
$$


b)

$$
\begin{aligned}
& B_{1 a^{\prime}}(x) \leqq L^{-\alpha / 3}\left(d\left(x, D_{\ell}\right)+1\right)^{-1}+L^{-\alpha} \text { for } a^{\prime}=5,6 \\
& B_{a a^{\prime}}(x) \leqq c L^{-\alpha} \text { for } a \neq 1, a^{\prime}=5,6
\end{aligned}
$$

c)

$$
B_{a a^{\prime}}(x) \leqq c L^{-\alpha+2 \beta} \quad \text { for } \quad a, a^{\prime}=5,6 .
$$

We may bound (70) in terms of $A$ 's and $B$ 's:

$$
(70) \leqq \sum_{k \geqq 2} e^{c k} \sum_{a} A_{a_{1}}\left(L x^{\prime}\right) \prod_{i \in I} \sup _{x} B_{a_{i} a_{i+1}}(x) \prod_{i \notin I} \sup _{x} A_{a_{i}}(x),
$$

where $I$ is the largest subset of $\{2, \ldots, k-1\}$ such that $\left(a_{i}, a_{i+1}\right) \neq(5,4),\left(a_{i}, a_{i+1}\right)$ $\neq(6,4) \forall i \in I$ and $|i-j| \geqq 2$ for $i, j \in I$. The reason behind this complicated definition is that (84) gives a bound $L^{-\alpha / 4}$ on each $B_{a_{i} a_{i+1}}(x)$ for $i \in I$. Also $|i-j| \geqq 2$ implies that pairs $(i, i+1),(j, j+1)$ are disjoint so that $(88)$ holds. Since only every other pair $\left(a_{i}, a_{i+1}\right)$ can be of the form $(5,4)$ or $(6,4)$ and since we get from Lemma 4 , sup $A_{a}(x)$ $\leqq c L^{\beta}$, for all $a$, and since $\beta \ll \alpha$, the sum over $k$ and $\underline{a}$ in $(88)$ is bounded by $c L^{\beta}$. However, to prove (68) we need to bound (88) by $\frac{1}{2} L^{-\alpha / 2}$. This requires a careful case by case analysis.

First, let us assume that $L x^{\prime} \notin D_{+}+B\left(L^{\alpha}\right)$. Since $D_{\ell} \subset D_{+}$, by Lemma 1 , e, we have

$$
d\left(L x^{\prime}, D_{\ell}\right) \geqq d\left(L x^{\prime}, D_{+}\right) \geqq L^{\alpha}
$$

and trivially, $\chi\left(L x^{\prime} \in D_{+} \backslash \bar{D}\right)=0$.

Using this and $(76,77,80,81)$, we get

$$
A_{a_{1}}\left(L x^{\prime}\right) \leqq L^{-\alpha} c L^{\beta}
$$

for all $a_{1}$. Inserting (90) in (88) gives the desired bound.

Now, let $L x^{\prime} \in D_{+}+B\left(L^{\alpha}\right)$, still with $x^{\prime} \notin D_{+}^{\prime}$. First, observe that, by (77), we are done if a single $a_{i}=2$ or 3 [we may modify (88) so as to put this $i$ in $I^{c}$ without spoiling the convergence]. So we assume from now on $a_{i} \neq 2,3$. If $a_{1}=5,6$ we may use (88) and (81) since $x^{\prime} \notin D_{+}^{\prime}$ implies that $d\left(L x^{\prime}, D_{\ell}\right) \geqq L^{\alpha}$ (see 8, 10-14, 7). If $a_{2}=5,6$ we may use (85) or (86): modify (88) so as to have $B_{a_{1} a_{2}}\left(L x^{\prime}\right)$ as the first factor and use $d\left(L x^{\prime}, D_{\ell}\right) \geqq L^{\alpha}$ in (85). Finally, if $a_{3}=5,6$ but $a_{1}, a_{2} \in\{1,4\}$, we consider several cases: if $a_{2}=4$, use (88) and (86) for $B_{4 a_{3}}$. If $a_{2}=1$ and $a_{1}=1$, use simply (76) for each $A$ factor:

$$
L^{-2 \alpha / 3} c L^{\beta} \ll L^{-\alpha / 2}
$$

So consider $a_{1}=4, a_{2}=1$, and $a_{3}=5,6$. If $t_{1} \neq 0$ we use (79), so, let $t_{1}=0$ ). We have to consider

$$
\int d x\left|Q_{4 Z}\left(L x^{\prime}, x\right)\right| B_{1 a^{\prime}}(x) e^{2 L^{-1} d_{4}\left(L x^{\prime}, x, Z\right)}
$$

with $a^{\prime}=5,6$.

We want to use $(85)$ and $d\left(L x^{\prime}, D_{\ell}\right) \geqq L^{\alpha}$ to control (92). So divide the integral in (92) into $\left|x-L x^{\prime}\right| \leqq L^{\gamma}$ and $\left|x-L x^{\prime}\right|>L^{\gamma}$. For $\left|x-L x^{\prime}\right| \leqq L^{\gamma}$ we have, $d\left(x, D_{\ell}\right) \geqq \frac{L^{\alpha}}{2}$ and we may use (85). The integral over $x$ is bounded by a constant using Lemma 4 . For $\left|x-L x^{\prime}\right|>L^{\gamma}$, Lemma 4 gives a bound $c \exp \left(-\mathcal{O}\left(L^{\gamma}\right)\right)$ :

$$
d_{4}\left(L x^{\prime}, x, Z\right)=d\left(x, D\left(L x^{\prime}\right)\right) \geqq L^{\gamma}-1
$$


because $\operatorname{diam}\left(D\left(L x^{\prime}\right)\right) \leqq 1$. To see this, use Lemma 1, $d$ and $e$; here, $x^{\prime} \notin D_{+}^{\prime}$ so if $L x^{\prime} \in D_{+}, L x^{\prime} \in D_{+} \cap L D_{-}^{\prime}$ and we may use Lemma 1 (if $L x^{\prime} \notin D_{+}, Q_{4 Z}=0$ ).

So the only cases to consider for $k \leqq 4$ in (88) are:

$$
\begin{aligned}
& k=2, a_{1}=1,4, \\
& k=3,\left(a_{1}, a_{2}\right)=(1,4),(4,1) \text { or }(44), \\
& k=4,\left(a_{1}, a_{2}, a_{3}\right)=(1,4,4),(4,1,4), \text { and }(4,4,4) .
\end{aligned}
$$

We rule out two $a_{i}=1$ because (76) gives bound $L^{-\alpha / 3}$ for each of them and we use (91). In a similar way, we may exclude $k \geqq 5$ : (84) gives a bound $L^{-\alpha / 3} L^{2 \beta}$ for each pair $\left(a_{1}, a_{2}\right)\left(a_{3}, a_{4}\right)$, provided $a_{1} \notin\{5,6\}$ for $i=1$ or 3 . But we have already discussed $a_{1}=5,6$, and $a_{3}=5,6$. So we turn to (93). Again observe that, if $a_{i}=4, t_{i}$ must be zero, otherwise we are done by (79). For these, we shall go back to (48) i.e. not put absolute values on $Q_{a z}$, as we did in (70).

Consider first $k=2, a_{1}=4, t_{1}=0$. We have

$$
\int d x Q_{4 Z}\left(L x^{\prime}, x\right) T^{L^{2}-1}\left(x, L y^{\prime}\right) \chi\left(\left|L x^{\prime}-x\right| \leqq L^{\gamma}\right)
$$

since, if $\left|L x^{\prime}-x\right|>L^{\gamma}$ we use Lemma 4 to get a bound $\exp \left(-\mathcal{O}\left(L^{\gamma}\right)\right.$. Now, use

$$
\int d x Q_{4 Z}\left(L x^{\prime}, x\right)=0
$$

to write (94) as

$$
\int d x Q_{4 Z}\left(L x^{\prime}, x\right)\left(T^{L^{2}-1}\left(x-L y^{\prime}\right)-T^{L^{2}-1}\left(L x^{\prime}-L y^{\prime}\right)\right) \chi\left(\left|L x^{\prime}-x\right| \leqq L^{\gamma}\right) .
$$

Then use the bound

$$
L^{3} \int d y^{\prime}\left|T^{t}\left(x-L y^{\prime}\right)-T^{t}\left(L x^{\prime}-L y^{\prime}\right)\right| e^{2 L^{-1}\left|x-L y^{\prime}\right|} \leqq c t^{-1 / 2} L^{\gamma}
$$

which holds for

$$
\left|L x^{\prime}-x\right| \leqq L^{\gamma}, \quad t \leqq L^{2}
$$

Inserting (96), for $t=L^{2}-1$, in (95) and controlling $Q_{4}$ with Lemma 4 gives the desired bound on (94). We proceed in the same way for $k=3,4$, and $a_{i}=4$, all $i$, when $t_{i}=0$, for all $i$ and use (79) otherwise.

Now consider $k=2, a_{1}=1$. We write, going back to (48),

$$
\begin{aligned}
\sum_{Z, t} \int d x d y(x \notin \bar{D}) T^{t}\left(L x^{\prime}-x\right) Q_{1 Z}(x, y) T^{L^{2}-t-1}\left(y-L y^{\prime}\right) \\
=\sum_{Z}\left(\sum _ { t \leqq \frac { L ^ { 2 } } { 2 } } \int d x d y \chi ( x \notin \overline { D } ) T ^ { t } ( L x ^ { \prime } - x ) Q _ { 1 Z } ( x , y ) \left(T^{L^{2}-t-1}\left(y, L y^{\prime}\right)\right.\right. \\
\quad-T^{\left.\left.L^{2-t-1}\left(z-L y^{\prime}\right)\right)\right)} \\
\left.\quad+\sum_{t>\frac{L^{2}}{2}} \int d x d y \chi(x \notin \bar{D}) T^{t}\left(L x^{\prime}-x\right) Q_{1 Z}(x, y) T^{L^{2}-t-1}\left(y-L y^{\prime}\right)\right)
\end{aligned}
$$

where $z$ is the first point (in some order) in $Z$. To get (97), we used $\int d y Q_{1 z}(x, y)=0$.

To control (97), we use (96) for the sum over $t \leqq L^{2} / 2$ and $|z-y| \leqq L^{\gamma}$. If $|z-y|>L^{\gamma}$, then $d_{1}(x, y, Z) \geqq L^{\gamma}$ and we can use Lemma 4 to get a $\exp \left(-L^{\gamma} / 2\right)$ 
bound. For $t>\frac{L^{2}}{2}$ we use $(75)$ and

$$
\sum_{t>\frac{L^{2}}{2}} t^{-3 / 2} \leqq c L^{-1}
$$

All other terms in (93) can be treated similarly since we may assume $t_{i}=0$ for $a_{i}=4$.

2) Proof of (69). First observe that, if $N\left(Y^{\prime}\right) \neq 0$, for each term in (48) contributing to $\tilde{\varepsilon}_{Y^{\prime}}$, i.e. satisfying $(52-54)$, we have $a_{i} \in\{1,3,4\}$ for all $i$. Indeed $a_{i}=2$ would mean $Z_{i} \cap \widetilde{D} \neq \phi$ for some $Z_{i}$ in $(52)\left(E_{Y}=0\right.$ if $\left.Y \cap \bar{D}=\phi\right)$. But $\widetilde{D} \subset D_{\ell} \subset L D_{+}^{\prime}$ by $(5,8,10-14)$. Similarly, if $a_{i}>4$, we have $W\left(y_{i}\right) \cap \bar{D} \neq \phi$ and $W\left(y_{i}\right)$ contributes to (52). But $\widetilde{D}^{\prime} \subset D_{\ell}^{\prime} \subset D_{+}^{\prime}$, hence $W\left(y_{i}\right) \cap \bar{D} \neq \phi$ implies $W\left(y_{i}\right) \cap L D_{+}^{\prime} \neq \phi$.

We have to bound $L^{-N\left(Y^{\prime}\right) / 2}$ in (69). For that, we use (10), which gives

$$
L^{-N\left(Y^{\prime}\right) / 2} \leqq L^{1-\alpha} \min _{a_{i} \neq 3} L^{-N\left(Z_{i}\right) / 2},
$$

where the minimum is taken over all $Z_{i}$ in (48) with $a_{i} \neq 3$. As we noticed after Eq. (50), there is always at least one $i$ with $a_{i} \neq 3$. Also, remember that, if $N\left(Y^{\prime}\right)=-\infty, \varepsilon_{Y^{\prime}}=0$ (by Proposition 1).

Using (48), (98), and Lemma 3, we get

$$
\begin{aligned}
(69) \leqq & \exp \left(-0\left(L^{\gamma}\right)\right)+L^{1-\alpha} \sum_{k \geqq 2} e^{c k} \sum_{\underline{t}} \sum_{a}^{*} \sum_{Z} \int d \underline{x} d \underline{y} \chi_{4} \prod_{i=1}^{k-1} T^{t_{i}}\left(x_{i-1}-y_{i}\right) \\
& \times e^{L^{-1}\left|x_{i}-y_{i}\right|} \prod_{i=1}^{k-1}\left|Q_{a_{i} Z_{i}}\left(y_{i}, x_{i}\right)\right| e^{2 L^{-1} d_{a_{i}}\left(y_{i}, x_{i}, z_{i}\right)} \prod_{\substack{i \\
a_{i} \neq 3}} L^{-N\left(Z_{i}\right) / 2} \\
& \times\left|T^{t_{k}}\left(x_{k-1}-y_{k}\right)-T^{t_{k}}\left(z_{k-1}-y_{k}\right)\right| e^{L^{-1}\left|x_{k-1}-y_{k}\right|} \chi\left(\left|x_{k-1}-z_{k-1}\right| \leqq L^{\gamma}\right),
\end{aligned}
$$

where $\sum_{a}^{*}$ means $a_{i} \in\{1,3,4\}$ all $i$, and we used: $\min _{i}-N\left(Z_{i}\right) \leqq-\sum_{i} N\left(Z_{i}\right)$, since $-N(Z) \geqq 0$. We also used $\int d x_{k-1} Q_{a Z_{k-1}}\left(y_{k-1}, x_{k-1}\right)=0$ in order to subtract $T^{t_{k}}\left(z_{k-1}-y_{k}\right)$, where $z_{k-1}$ is the first point of $Z_{k-1}$. The term $\exp \left(-\mathcal{O}\left(L^{\gamma}\right)\right)$ comes from

$$
d_{a}\left(x_{k-1}, y_{k-1}, Z_{k-1}\right) \geqq\left|x_{k-1}-y_{k-1}\right| \geqq L^{\gamma}-1
$$

for $a=1,3,4$. For $a=4$, we use $Z_{k-1}=D\left(y_{k-1}\right)$ and $\operatorname{diam}\left(D\left(y_{k-1}\right)<1\right.$, which follows from Lemma $1 \mathrm{~d}$, e and the fact that $\chi_{4}$ in (99) means $y_{k-1} \in D_{+} \cap L D^{\prime}$ for $a_{k-1}=4[$ see (70) and (31)].

To bound (99) by $\frac{L^{-\alpha / L}}{2}$ we use Lemma 5 : we may combine $(74,75,77,79)$ into

$$
A_{a}(x, t) \leqq L^{-\alpha / 3}(t+1)^{-3 / 2}
$$

for $t \leqq L^{2}$, and $a \in\{1,3,4\}$ unless $a=4$ and $t=0$, for which we have only (78). But if $a_{i}=4, a_{i+1}=4$ we may use (84) to obtain a small bound. So, since the $\underline{t}$ and $\underline{Z}$ sums in (99) can be bounded by products of $A_{a}$ 's and $B_{44}$ 's when $a_{i}=a_{i+1}=4$, $t_{i}=t_{i+1}=0$, we get

$$
(99) \leqq L^{1-\alpha} \sum_{k \geqq 2} e^{c k} L^{-\alpha(k-2) / 6}\left(c L^{\gamma}\right) \sum_{t, \Sigma t_{l}=L^{2}-k-1} \prod_{i=1}^{k-1}\left(t_{i}+1\right)^{-3 / 2}\left(t_{k}+1\right)^{-1 / 2}
$$


where we used (96) for the $y_{k}$ integral. The sum over $\left(t_{i}\right)_{i=1}^{k}$ is less than $\left(\left(L^{2}-k-1\right)^{1 / 2}+1\right)^{-1}$. Then the $k$ sums is bounded by $c L^{-1}$ and we have finally (69) $\leqq(99) \leqq(101) \leqq c L^{-\alpha} L^{\gamma} \leqq \frac{L^{-\alpha / 2}}{2}$ which finishes the proof of (69).

B. Proof of (26) for $x^{\prime} \notin D_{+}^{\prime}$. The proof uses the large corridors $B\left(2 L^{1+\gamma}\right)$ in $L D_{\ell}^{\prime}$ [see (5)] and the fact that, by (55), $\widetilde{E}_{Y}^{\prime}$ collects only terms which have to "cross" these corridors and are thus small due to exponential decay.

Fix a term in (48) contributing to $\widetilde{E}_{Y^{\prime}}$. Let

$$
J=\left\{i \in\{1, \ldots, k-1\}, a_{i}>4, b_{i}=2\right\} \cup\{0\} .
$$

Then we have

Lemma 7. a) Let $j \in J, j \neq 0$, and $j^{\prime}=\max _{\substack{k<k \\ k \in J}} k$. Then,

$$
\sum_{i=j^{\prime}}^{j-1} d_{a_{i}}\left(y_{i}, x_{i}, Z_{i}\right)+\sum_{i=j^{\prime}+1}^{j}\left|x_{i-1}-y_{i}\right| \geqq \frac{3}{2} L^{1+\gamma}
$$

b) For $a=2,5, y \notin L \tilde{D}^{\prime}$ and $Z_{D} \neq \phi$ (see (53)), $d_{a}(y, x, Z) \geqq \frac{L}{2}$.

Using (48), Lemma $3 b$ and Lemma 7, we get

$$
\begin{aligned}
\sum_{Y} \int & d y\left|\widetilde{E}_{Y}(x, y)\right| e^{d\left(x \cup y \cup Y, Y \cap D_{t}^{\prime}\right)} \leqq 2 \sum_{k \geqq 2} e^{c k} \sum_{\underline{t} t^{\prime}} \sum_{a b} \sum_{Z} \exp \left(-\frac{3}{2} L^{\gamma}\left(n_{2}+m(\underline{Z})\right)\right) \\
\quad & \times \int d \underline{x} d \underline{y} \chi_{5} \prod_{i=1}^{k} T^{t_{i}}\left(x_{i}-y_{i}\right) e^{2 L^{-1}\left|x_{i}-y_{t}\right|} \\
& \times \prod_{i=1}^{k-1}\left|Q_{a_{i} z_{i}}^{t_{i}}\left(y_{i}, x_{i}\right)\right| e^{d_{a_{i}}\left(x_{i}, y_{i}, Z_{i}\right) / 4}+|(b \rightarrow s)|
\end{aligned}
$$

where

$$
\begin{gathered}
n_{2}=\left|\left\{i \mid a_{i}=5,6, b_{i}=2\right\}\right|, \\
m(\underline{Z})=\left|\left\{i \mid a_{i}=2,5, Z_{i D} \neq \phi\right\}\right|
\end{gathered}
$$

and

$$
\begin{aligned}
\chi_{5}= & \prod_{i}^{\prime} \chi 4\left(x_{i} \notin \bar{D}\right) \chi\left(y_{i} \notin \bar{D}\right) \prod_{\substack{a_{i}>4 \\
b_{i}=1}} \chi\left(y_{i} \in \bar{D} \backslash L \bar{D}^{\prime}\right) \\
& \times \prod_{\substack{a_{i}>4 \\
b_{i}=2}} \chi\left(y_{i} \in L \widetilde{D}^{\prime}\right) \prod_{a_{i}>4}^{\prime} \chi\left(x_{i} \notin W\left(y_{i}\right)\right) .
\end{aligned}
$$

The constraint (55) means that $n_{2}+m(Z) \geqq 1$ for each term in (103). Thus we get a prefactor $\exp \left(-L^{\gamma}\right)$ which gives the upper bound in (26) and all we need to do is to bound the sum. For this, we use Lemma 5 and 6 as in the bound on (88); we need a bound on the factors which are similar to $A_{a}$ with $a=5,6$ [see (73)] but with $b_{i}=2$. We have, using the remaining factor $\exp \left(-\frac{L^{y} n_{2}}{2}\right)$ in (103):

$$
\begin{aligned}
& e^{-\frac{L^{\gamma}}{2}} \sum_{t, t^{\prime}} \sum_{Z} \int d y d z \chi\left(y \in L \tilde{D}^{\prime}\right) \chi(z \notin W(y)) T^{t}(x-y) \\
& \quad \times e^{2 L^{-1}|x-y|}\left|Q_{a Z}^{t^{\prime}}(y, z)\right| e^{d_{a}(x, y, Z) / 4} \leqq c L^{2} e^{-L^{\gamma / 2}} \leqq e^{-L^{\gamma / 4}}
\end{aligned}
$$


using Lemma 4 for the $z$ integral and for the $Z$ and $t^{\prime}$ sums, and bounding by $L^{2}$ the sum over $t$. The $y$ integral is bounded by a constant, because $T$ has exponential decay. This concludes the proof of (26) for $x^{\prime} \in D_{+}^{\prime}$.

$C$. Proof of (25-27) for $x^{\prime} \in D_{+}^{\prime}$. We use (48), modified for $x^{\prime} \in D_{+}^{\prime}$, and the corridor $B\left(2 L^{\gamma}\right)$ in $(57,59)$. First consider $(27)$. We can use Lemma $3 \mathrm{c}$ to bound

$$
\begin{aligned}
\int d y^{\prime}\left|\ell^{\prime}\left(x^{\prime}, y^{\prime}\right)\right| e^{d\left(y^{\prime}, D\left(x^{\prime}\right)\right)} \leqq & \sum_{Z} \int d x_{1}\left|Q_{5 Z}\left(L x^{\prime}, x_{1}\right)\right| e^{2 L^{-1} d_{5}\left(L x^{\prime}, x_{1}, Z\right)} \\
& \times \chi\left(x_{1} \notin W_{1}\right) \int c\left(x_{1}, y\right) d y
\end{aligned}
$$

where $\int c\left(x_{1}, y\right) d y$ is bounded by one plus a sum of products of $A$ 's and $B$ 's, as in (88). To get (105), we use $t_{1}=0, a_{1}=5$ in the modified (52). Also, in (105), $W_{1}=W\left(L x^{\prime}\right)$ $=L D^{\prime}\left(x^{\prime}\right)$. Divide the $x_{1}$ integral into $d\left(x_{1}, W\left(L x^{\prime}\right)\right) \leqq L^{\gamma}$ and $d\left(x_{1}, W\left(L x^{\prime}\right) \geqq L^{\gamma}\right.$.

In the first case we have $d\left(x_{1}, D_{+}\right) \geqq L^{\alpha} / 2$ because $L D^{\prime}\left(x^{\prime}\right)$ is connected and there is a corridor of size $L^{\alpha}$ in (6) around $D_{+}$. This and $d\left(x_{1}, D_{\ell}\right) \geqq d\left(x_{1}, D_{+}\right)$, see (89), inserted in (76), (80), (81), allows us to bound $\int c\left(x_{1}, y\right) d y$ in (105), using Lemma 5 and 6, by $0(1)$. Then, Lemma 4 controls the $x_{1}$ integral and the sum over $Z$ in (105).

For $d\left(x_{1}, W\left(L x^{\prime}\right)\right) \geqq L^{\gamma}$, we get a bound $0\left(L^{\beta}\right)$ on $\int c\left(x_{1}, y\right) d y$, from Lemma 5 and 6 , but a factor $e^{-c L^{\gamma}}$ from Lemma 4 , since, then, $d_{5}\left(L x, x_{1}, Z\right) \geqq L^{\gamma}$, again because $d\left(\partial W(L x), D_{\ell}\right) \geqq d\left(\partial W(L x), D_{+}\right) \geqq L^{\alpha}$.

The proof of $(25-26)$ is similar to the one of $(26)$ for $x^{\prime} \in D_{+}^{\prime}$, using the following lemma.

Lemma 8. Let $x^{\prime} \in D_{+}^{\prime}$. Then, for each term of the modified (48) contributing to $\tilde{\varepsilon}+\tilde{E}$, i.e. such that (59) holds,

$$
\sum_{1}^{k}\left|x_{i-1}-y_{i}\right|+\sum_{1}^{k} d_{a_{i}}\left(y_{i}, x_{i}, Z_{i}\right) \geqq \frac{3}{2} L^{1+\gamma} .
$$

\section{Reabsorption of the Traps}

Here, we define inductively $\Delta s$, see $(17,24,33)$ below, and show that it satisfies $(5.68)$ (and Proposition 5.2). Moreover, its definition will imply that $\varepsilon_{Y}=0$ when $N(Y)=-\infty$, as we claimed in Proposition 6.1.

Let now

$$
L^{\frac{1}{2} N(Y)} \leqq \delta^{2}
$$

i.e.

$$
N(Y) \leqq-2 n(1-2 \alpha) .
$$

This means, in particular, that

$$
\int_{\mathbf{v}}\left|\varepsilon_{Y}(x, y)\right| d y \leqq L^{\frac{1}{2} N(Y)} \zeta^{|Y|} e^{-d(x \cup v \cup Y)}
$$

for $\zeta=L^{-\alpha}$ if (2) holds. To get (3) from (6.25), note that, if (2) holds for $Y^{\prime}$, then only $\varepsilon_{Y}$ with $Y \cap D_{\ell}=\phi$ and $s_{Y}$ are involved in the expansion (6.48) for $\varepsilon$ :

$$
\varepsilon_{Y^{\prime}}^{\prime}\left(x^{\prime}, y^{\prime}\right)=L^{3} \sum_{n, Y} \int d \omega_{L x^{\prime} L y^{\prime}}^{L^{2}} \prod T^{n_{i}}\left(\varepsilon_{Y_{i}}+s_{Y_{i}}\right)-(\varepsilon=0) \text { - average . }
$$

Moreover, for $u^{\prime} \in Y^{\prime}$ such that $N_{u^{\prime}}^{\prime} \neq-\infty, N_{u^{\prime}}^{\prime} \leqq 0$ we know that (see Lemma 6.1) 
$u^{\prime} \notin \widetilde{D^{\prime}},\left|L \mathbf{u}^{\prime} \cap \mathbf{D}\right| \leqq c L^{\beta}$ and

$$
N_{u^{\prime}}^{\prime}=\max _{u \in L \mathbf{u}^{\prime}} N_{u}-2(1-\alpha)
$$

Thus, from (6.10)

$$
\int_{\mathbf{v}}\left|\varepsilon_{Y_{i}}(x, y)\right| d y \leqq c L^{\frac{1}{2} N^{\prime}\left(Y^{\prime}\right)+(1-\alpha)} L^{-\alpha / 2} e^{-d\left(x \cup v \cup Y_{\imath}\right)} .
$$

Now we may estimate (4) as we estimated $s_{Y}$ in Sect. 5 . The linear term in $\varepsilon$,

$$
(\mathscr{L} \varepsilon)_{Y^{\prime}}\left(x^{\prime}, y^{\prime}\right)=L^{3} \sum_{t} \sum^{\prime} \int T^{t}\left(L x^{\prime}-x\right) \varepsilon_{Y}(x, y) T^{L^{2}-t-1}\left(y, L y^{\prime}\right),
$$

where $\Sigma^{\prime}$ is as in $(5.70,5.71)$, once integrated, has the bound

$$
e^{-C L\left|Y^{\prime}\right|} L^{\frac{1}{2} N^{\prime}\left(Y^{\prime}\right)} e^{-d\left(x^{\prime} \cup v^{\prime} \cup Y^{\prime}\right)}
$$

if $\left|Y^{\prime}\right|>1$, where $e^{-C L\left|Y^{\prime}\right|}$ comes from the scaling. For $\left|Y^{\prime}\right|=1$, we obtain the bound

$$
C L^{-1} L^{\frac{1}{2} N^{\prime}\left(Y^{\prime}\right)+1-\alpha} L^{-\alpha / 2} e^{-d\left(x^{\prime} \cup v^{\prime} \cup Y^{\prime}\right)} \sum_{Y} e^{-\frac{1}{4} d(Y)}
$$

with $Y \cap D \neq \phi,\left[L^{-1} Y\right]=Y^{\prime}$. The $Y$-sum is bounded by $C L^{\beta}((9)$ is very generous: actually the term in (7) having only $L^{-1}$ is the one with $t=0$, which has the factor $e^{-d\left(L x^{\prime} \cup Y\right)}$ to control $\left.\sum_{Y}\right)$. Equations (8) and (9) yield (3).

Now we want to show that, as $N$ decreases from (2), the RGT brings additional contraction, enabling us to gradually reach the Fourier bounds valid for $s_{Y}$. Looking at (4), we see that terms where $n_{0}>0$ or $n_{k}>0$ automatically satisfy our Fourier bounds at least in $\xi_{1}$ or $\xi_{2}$. The same holds for terms with $s_{Y_{i}}$ at the end or the beginning of the walk $\omega$. The other terms have $\varepsilon$ 's tied to the end or beginning of $\omega$, and will contract strongly under $\mathscr{R}$. This motivates us to split $\varepsilon$ in three pieces, defined below, depending on where Fourier-bounds with $A(k)^{2 / 3}$ or $A(k)^{1 / 3}$ hold:

$$
\varepsilon_{Y}=\varepsilon_{1 Y}+\varepsilon_{2 Y}+\varepsilon_{3 Y} .
$$

Let us introduce a convenient index counting how much smaller than (2) $N$ is: put

$$
-\alpha \ell(Y)=\frac{1}{2} N(Y)+n(1-2 \alpha)
$$

i.e.

$$
L^{\frac{N}{2}}=L^{-\alpha \ell} \delta^{2}
$$

Then we have, for $Y$ as in (2), that $\varepsilon_{i Y}$ satisfy (3) and $(\ell=\ell(Y))$

$$
\begin{gathered}
\int_{\mathbf{v}_{1} x \mathbf{v}_{2}}\left|\varepsilon_{1 Y}(x, y)\right| d x d y \leqq c \delta^{4} L^{-(3+\alpha)(\ell-1)} e^{-d\left(v_{1} \cup v_{2} \cup Y\right) \zeta|Y|}, \\
\int_{\mathbf{v}_{2}}\left|\varepsilon_{2 Y}(\xi, y)\right| d y \leqq c \delta^{2} L^{-(1+\alpha) \ell} A(k)^{2 / 3} e^{-d\left(v_{1} \cup v_{2} \cup Y\right) \zeta|Y|}, \\
\int_{\mathbf{v}_{1}}\left|\varepsilon_{3 Y}(x, \xi)\right| d x \leqq c \delta^{2} L^{-(2+\alpha) \ell} A(k)^{1 / 3} e^{-d\left(v_{1} \cup v_{2} \cup Y\right)} \zeta^{|Y|}
\end{gathered}
$$


To prove (13)-(15) iteratively is now only a matter of book-keeping. To define $\varepsilon_{i}$ from $\varepsilon$, let $\ell^{\prime}=0$ and note, first, that all the terms $t$ of the form $(T+s)$ (anything) $(T+s)$ have

$$
\left\|t_{Y}\right\|_{i} \leqq c \delta^{2} \zeta^{|Y|}
$$

due to the bound (3) [recall (1)] and the fact that at least one $\varepsilon$ has to occur in the (anything). Thus, we put

$$
\begin{gathered}
\Delta \mathrm{s}_{Y^{\prime}}^{\prime}=L^{3}\left[\int d \omega\left[(T+s)(T+b)^{L^{2}-2}(T+s)-(T+s)^{L^{2}}\right]\right]_{Y^{\prime}} \text { - average, } \\
\varepsilon_{1 Y^{\prime}}^{\prime}=L^{3}\left[\int d \omega\left[\varepsilon(T+b)^{L^{2}-2} \varepsilon\right]\right]_{Y^{\prime}} \text {-average, } \\
\varepsilon_{2 Y^{\prime}}^{\prime}=L^{3}\left[\int d \omega\left[(T+s)(T+b)^{L^{2}-2} \varepsilon\right]\right]_{Y^{\prime}} \text {-average, } \\
\varepsilon_{3 Y^{\prime}}^{\prime}=L^{3}\left[\int d \omega\left[\varepsilon(T+b)^{L^{2}-2}(T+s)\right]\right]_{Y^{\prime}} \text { - average }
\end{gathered}
$$

with obvious notation: we expand $b=s+\varepsilon$ and collect terms as in $(5.70,5.71)$. The bounds (13)-(15) for $\ell=0$ follow readily from the bound (3) for $\varepsilon$, see also below. To iterate (13)-(15), let $\ell^{\prime}\left(Y^{\prime}\right)>0$. We put

$$
\begin{gathered}
\varepsilon_{1 Y^{\prime}}^{\prime}=L^{3}\left[\int d \omega\left(\varepsilon_{1}+\varepsilon_{3}\right)(T+b)^{L^{2}-2}\left(\varepsilon_{1}+\varepsilon_{2}\right)\right]_{Y^{\prime}}-\text { av. } \\
\varepsilon_{2 Y^{\prime}}^{\prime}=L^{3}\left[\int d \omega\left(\varepsilon_{2}(T+b)^{L^{2}-1}+(T+s)(T+b)^{L^{2}-2}\left(\varepsilon_{1}+\varepsilon_{2}\right)\right)\right]_{Y^{\prime}}-\text { a.v. } \\
\varepsilon_{3 Y^{\prime}}^{\prime}=L^{3}\left[\int d \omega\left(\varepsilon_{1}+\varepsilon_{3}\right)(T+b)^{L^{2}-2}\left(T+s+\varepsilon_{3}\right)\right]_{Y^{\prime}}-\text { av. } \\
\Delta s_{Y^{\prime}}^{\prime}=(17)+L^{3}\left[\int d \omega(T+s)(T+b)^{L^{2}-2} \varepsilon_{3}\right]_{Y^{\prime}}-\text { av. }
\end{gathered}
$$

$\Delta s_{Y^{\prime}}^{\prime}$ satisfies (16) again, $\varepsilon_{1 Y^{\prime}}^{\prime}$ has as leading term $\varepsilon_{3}(T+b)^{L^{2}-2} \varepsilon_{2}$, which is $\varepsilon_{1}$-independent, so (13) holds.

$$
\begin{aligned}
\left(\mathscr{L}_{2}\right)_{Y^{\prime}}\left(x^{\prime}, y^{\prime}\right)= & L^{3} \sum_{Y} \int d x \varepsilon_{2 Y}\left(L x^{\prime}, x\right)\left(T^{L^{2}-1}\left(x-L y^{\prime}\right)-T^{L^{2}-1}\left(u-L y^{\prime}\right)\right) \\
& +L^{3} \sum_{Y} \int d x T^{L^{2}-1}\left(L x^{\prime}-x\right) \varepsilon_{2 Y}\left(x, L y^{\prime}\right),
\end{aligned}
$$

where $\int \varepsilon_{Y}(x, y) d y=0$ was used. Also, for each $Y \subset Z^{d}$ choose a preferred point $u$. Using $\varepsilon_{2 Y}\left(L x^{\prime}, x\right)$ and

$$
\begin{aligned}
& \left|T^{L^{2}-1}\left(x-L y^{\prime}\right)-T^{L^{2}-1}\left(u-L y^{\prime}\right)\right| \leqq C L^{-4} e^{-2\left|y^{\prime}-\frac{x}{L}\right|+\frac{2}{L}|x-u|} \\
& \times\left|L^{3} T^{L^{2}-1}(L \cdot-\cdot)\left(\xi_{1}^{\prime}, \xi_{2}\right)\right| \\
& \leqq C e^{-2\left|v_{1}-\frac{v_{2}}{L}\right|} \int d q a\left(\frac{q}{L}\right)^{L^{2}-1} A\left(k_{1}^{\prime}-q\right) A\left(k_{2}+\frac{q}{L}\right),
\end{aligned}
$$

we get, with similar estimates as in Sect. 5 (see proof of Lemma 5.1),

$$
\begin{aligned}
\int_{\mathbf{v}_{2}^{\prime}}\left|\left(\mathscr{L} e_{2}\right)_{Y^{\prime}}\left(\xi^{\prime}, y^{\prime}\right)\right| d y^{\prime} \leqq & C\left(L^{-2}+L^{-3}\right) \delta^{2} A\left(k^{\prime}\right)^{2 / 3} e^{-d\left(v_{1}^{\prime} \cup v_{2}^{\prime} \cup Y^{\prime}\right) \zeta\left|Y^{\prime}\right|} \\
& \times \sum_{Y} L^{-(1+\alpha) \ell(Y)} e^{-\frac{1}{2} d(Y)},
\end{aligned}
$$

where $Y \cap D \neq \phi$. Now (5) and (11) give

$$
\ell(Y) \geqq \ell^{\prime}\left(Y^{\prime}\right)-1,
$$

which, with

$$
\sum_{Y} e^{-\frac{1}{2} d(Y)} \leqq C L^{\beta}
$$


let us conclude

$$
(27) \leqq C L^{-\alpha+\gamma} \delta^{\prime 2} L^{-(1+\alpha) \ell^{\prime}\left(Y^{\prime}\right)} A\left(k^{\prime}\right)^{2 / 3} \zeta^{\left|Y^{\prime}\right|} e^{-d\left(v_{1}^{\prime} \cup v_{2}^{\prime} \cup Y^{\prime}\right)} .
$$

Equation (30) together with the much smaller nonlinear terms in (22) $(\varepsilon$ is also negligible) gives (14).

To prove (15), consider the linear part of (23),

$$
\left(\mathscr{L} \varepsilon_{3}\right)_{Y^{\prime}}\left(x^{\prime} y^{\prime}\right)=L^{3} \sum_{Y} \int d x \varepsilon_{3 Y}\left(L x^{\prime}, x\right) T^{L^{2}-1}\left(x-L y^{\prime}\right) .
$$

This is readily bounded by

$$
\int_{\mathbf{v}_{2}}\left|\left(\mathscr{L}_{\varepsilon_{3}}\right)_{Y^{\prime}}\left(x^{\prime}, \xi^{\prime}\right)\right| d x^{\prime} \leqq C L^{-3} \delta^{2} \zeta^{|Y|} A\left(k^{\prime}\right)^{1 / 3} e^{-d\left(v_{1} \cup v_{2} \cup Y^{\prime}\right)} \sum_{Y} L^{-(2+\alpha) \ell(Y)} e^{-\frac{1}{2} d(Y)},
$$

which gives $C L^{-\alpha+\beta}$ times the bound (15). The nonlinear terms can be absorbed into this and (15) is proven.

Finally, if $L^{-(2+\alpha) \ell(Y)} \leqq L^{-2 n}$ we have

$$
L^{-(2+\alpha) \ell(Y)} \leqq A(k)^{2 / 3} \quad \text { and } \quad L^{-(1+\alpha) \ell(Y)} \leqq A(k)^{1 / 3},
$$

and $\varepsilon_{Y}$ satisfies the $\Delta s$-bounds:

$$
\Delta s_{Y}=\varepsilon_{Y} .
$$

Note, that this happens (see 11) if $-\frac{2 \alpha n}{2+\alpha} \geqq \frac{1}{2} N(Y)+n(1-2 \alpha)$ i.e. when $N(Y)$ $\leqq-2 n\left(1-2 \alpha+\frac{2 \alpha}{2+\alpha}\right)$, which proves that $\varepsilon_{Y}=0$ when $N(Y)=-\infty$.

\section{Traps - Lower Bounds}

Our goal here will be to prove (6.28). This will be a consequence of the following lower bounds on $T+b$ :

Proposition 1. There exists a constant $c_{1}$ such that, for all $u \in \mathbf{Z}^{d}$,

$$
\min _{|v-u|=1} \min _{x \in \mathbf{u}} \int_{\hat{\mathbf{v}}} d y(T+b)(x, y) \geqq c_{1} \beta_{u},
$$

where

and

$$
\begin{aligned}
\hat{\mathbf{v}} & =\mathbf{v} \text { if } v \in D_{\ell} \\
& =\mathbf{v} \backslash D \text { if } v \notin D_{\ell}
\end{aligned}
$$

$$
\beta_{u}=L^{2-N_{u}} / 1+L^{2-N_{u}},
$$

with $N_{u}$ defined in (6.14). Moreover, if $n_{u}=0$ (see (6.8)), then

$$
\min _{x \in \mathbf{u}} \int_{D^{c}} d y(T+b)(x, y) \geqq\left(1-\frac{1}{2} L^{-\frac{\alpha}{3}}\right) \beta_{u} .
$$

The only goal of this section is to prove (6.28). This will use (4), while (1) is only used to derive (4). As one sees, (1) gives a lower bound on the probability to go to an adjacent block, while, in (4), one controls the probability to escape from $D$. The 
main distinction in the proof will be between $n_{u}=0$ and $n_{u}=1$. In the latter case, we can only prove (1); that is the region where $D$ is dense, and all we use is a kind of lower bound through ballistic motion. When $n_{u}=0$, we may use the fact that $D$ is sparse so that, once one escapes from $D$, the probability of returning to it is small, see $(23,25)$. The flow of $\beta_{u}$ was discussed in Sect. 3 , for $\beta_{u}$ small; the denominator in (3) gives the correct behaviour for $\beta_{u}$ close to 1 .

Now, we shall prove (6.28), using (4). We write $\ell=(T+b)-T-s-e$, so,

$$
|\ell(x, y)| \leqq(T+b)(x, y)+T(x-y)+|s(x, y)|+|e(x, y)| .
$$

Since $\int d y(T+b)(x, y)=1$, we have, by $(4)$,

$$
\int_{D_{s} \backslash L \hat{D}^{\prime}}(T+b)(x, y) \leqq \int_{D} d y(T+b)(x, y)=1-\int_{D^{c}} d y(T+b)(x, y) \leqq 1-\beta_{u}+\frac{1}{2} L^{-\frac{\alpha}{3}},
$$

since $\beta \leqq 1$, by (3),

$$
\int_{D_{s} \backslash L \hat{D}^{\prime}} d y T(x-y)=\sum_{u^{\prime} \notin \hat{D}^{\prime}} \int_{D_{s} \cap L \mathbf{u}^{\prime}} d y T(x-y) \leqq \sum_{u^{\prime} \notin \hat{D}^{\prime}} e^{-\left|x-L u^{\prime}\right|}\left|D_{s} \cap L \mathbf{u}^{\prime}\right| \leqq c L^{-\alpha} .
$$

Using Lemma 6.1c. Finally,

$$
\int d y(|e(x, y)|+|s(x, y)|) \leqq 2 L^{-\alpha / 2} .
$$

This follows from (6.25), (6.26), and (5.11). Combining (5-8) proves (6.28).

Proof of Proposition 1. We proceed by induction: (1) holds for $n=n_{0}$ by (3.76) $\left(D=D_{\ell}\right.$ for $\left.n=n_{0}\right)$. We shall first prove (1) with primes when $n_{u^{\prime}}=1$. Choose a selfavoiding nearest neighbor walk $\omega$ on $\mathbf{Z}^{d}$, with $|\omega|=L^{2}, L x^{\prime} \in \omega(0), \omega(i) \subset L \mathbf{u}^{\prime}, \omega\left(\mathbf{L}^{2}\right)$ $C L \hat{\mathbf{y}}^{\prime}$. This is possible, since $\left|u^{\prime}-v^{\prime}\right|=1, L \mathbf{u}^{\prime}$ contains $L^{3} \gg L^{2}$ sites and there are at least $L^{2}-c L^{3 \alpha} L^{\beta}$ sites in $L \hat{\mathbf{v}}^{\prime}$ that are adjacent to $L \mathbf{u}^{\prime}$ if $\mathbf{v}^{\prime} \notin D_{\ell}^{\prime}$ (by construction of $\left.D^{\prime}\right)$. By definition,

$$
\int_{\hat{\mathbf{v}}^{\prime}} d y^{\prime}\left(T^{\prime}+b^{\prime}\right)\left(x^{\prime}, y^{\prime}\right)=\int_{L \mathbf{v}^{\prime}} d y^{\prime} \int d \omega_{L x^{\prime} y}^{L^{2}}(T+b)(\omega)
$$

and this can be bounded by:

$$
\geqq \prod_{i=0}^{L^{2}-1} \min _{x \in \omega(i)} \int_{\omega(i+1)} d y(T+b)(x, y)
$$

using the positivity of $(T+b)(x, y)$.

Using (1) inductively, since $|\omega(i)-\omega(i+1)|=1$,

$$
(10) \geqq c_{1}^{L^{2}} \prod_{i=0}^{L^{2}-1} \beta_{\omega(i)} \text {. }
$$

Using

$$
\beta_{u} \geqq \frac{1}{2} L^{-N_{u}}
$$

for $N_{u} \geqq 0$, and

$$
\beta \geqq \frac{1}{2} \text { for } \quad N_{u}<0
$$

we have

$$
(11) \geqq\left(\frac{c_{1}}{2}\right)^{L^{2}} \prod_{\mathbf{u} \subset L \mathbf{u}^{\prime}, N_{u}>0} L^{-N_{u}}
$$


where we used the fact that $\omega$ is self-avoiding. From (3) and (6.10-6.14) we have (for $\left.n_{u^{\prime}}=1\right)$

$$
(14) \geqq L^{2-N_{u^{\prime}}^{\prime}} \geqq c_{1} \beta_{\mathfrak{u}^{\prime}}^{\prime}
$$

for any $c_{1} \leqq 1$ and a suitable choice of $c_{0}$ in (6.10) depending only on $L$. The choice of $c_{1}<1$ will come from the proof of (1) when $n_{u^{\prime}}=0$, to which we now turn.

If $n_{u^{\prime}}=0$, it means [see (6.8), (6.5)] that

$$
L \mathbf{u}^{\prime} \cap\left(\left(L \tilde{D}^{\prime} \cup L R^{\prime}\right)+B\left(3 L^{1+\gamma}\right)\right)=\emptyset
$$

and

$$
L \mathbf{u}^{\prime} \cap\left(D_{\ell}+B\left(L^{\alpha}\right)\right)=\emptyset .
$$

We bound (9) from below by

$$
\min _{x^{\prime} \in \mathbf{u}^{\prime}} b\left(x^{\prime}\right) c\left(u^{\prime}, v^{\prime}\right)
$$

where

$$
b\left(x^{\prime}\right)=\sum_{t=1}^{\frac{L^{2}}{2}} \int_{S_{w}} d y \int d \omega_{L x^{\prime} y}^{t} \prod_{i=1}^{t-1} \chi(\omega(i) \in \mathbf{w} \cap D)(T+b)(\omega)
$$
where $\mathbf{w}=$ unit block containing $L x^{\prime}, S_{w}=\left\{y \notin \mathbf{w} \cap D,|y-w| \leqq \frac{L^{\alpha}}{2}\right\}$, the product
equals one for $t=1$, and

$$
c\left(u^{\prime}, v^{\prime}\right)=\min _{x^{\prime} \in \mathbf{u}^{\prime}, y \in S_{w}} \min _{t \leqq L^{2} / 2} \int_{L \hat{\mathbf{v}}^{\prime}} d y^{\prime} \int d \omega_{y y^{\prime}}^{L^{2}-t}(T+b)(\omega) \chi(\omega \cap D=\phi) .
$$

To prove (18), we write, for $y^{\prime} \notin \mathbf{w} \cap D$,

$$
\begin{aligned}
& \int d \omega_{L x^{\prime} y^{\prime}}^{L^{2}}(T+b)(\omega) \geqq \sum_{t=1}^{\frac{L^{2}}{2}} \int_{S_{w}} d y \int^{d} \omega_{y y^{\prime}}^{L^{2}-t}(T+b)(\omega) \\
& \quad \times \int d \omega_{L x^{\prime} y}^{t}(T+b)(\omega) \prod_{i=1}^{t-1} \chi(\omega(i) \in \mathbf{W} \cap D) .
\end{aligned}
$$

This means that we keep only, in the right-hand side, those $\omega$ 's which leave $\mathrm{w} \cap D$ before time $\frac{L^{2}}{2}$. We sum over the first exit time $t$ (these are disjoint events) and, furthermore, we keep only the walks where the step which leaves $\mathbf{w} \cap D$ does not go at a distance from $w$ larger than $\frac{L^{\alpha}}{2}$. To get (18), we take also the minimum over $t \leqq \frac{L^{2}}{2}$ and $y \in S_{w}$ to factorize (21) and insert $1 \geqq \chi(\omega \cap D=\phi)$ in the lower bound. To go from (18) to (1) we shall use the

Lemma. There exists a constant $c_{1}$ such that, if $n_{u^{\prime}}=0$ and $L x^{\prime} \in \mathbf{w}$,

$$
b\left(x^{\prime}\right) \geqq\left(1-e^{-c L^{\alpha}}\right) \frac{c_{1} \frac{L^{2}}{2} \beta_{w}}{1+\frac{c_{1} L^{2}}{2} \beta_{w}}
$$


and

$$
c\left(u^{\prime}, v^{\prime}\right) \geqq 2 c_{1} .
$$

To prove (1), insert (22), (23) in (18). Then, we use,

$$
\frac{c_{1} \frac{L^{2}}{2} \beta_{w}}{1+c_{1} \frac{L^{2}}{2} \beta_{w}}=\frac{c_{1} \frac{L^{2}}{2} L^{2-N_{w}}}{1+L^{2-N_{w}}\left(c_{1} \frac{L^{2}}{2}+1\right)} \geqq\left(1-L^{-2+2 \alpha}\right) \min _{w \in L \mathbf{u}^{\prime}} \frac{L^{2-2 \alpha} L^{2-N_{w}}}{1+L^{2-2 \alpha} L^{2-N_{w}}},
$$
which follows from $c_{1} \frac{L^{2}}{2}+1 \geqq L^{2-2 \alpha}$ and the fact that $c \rightarrow \frac{c L^{-N}}{1+c L^{-N}}$ is increasing
in $c$.

Since $N \rightarrow \frac{c L^{-N}}{1+c L^{-N}}$ is decreasing in $N$, we get from (6.10)-(6.14) that

$$
(24) \geqq\left(1-L^{-2+2 \alpha}\right) \beta_{u^{\prime}}^{\prime}
$$

because $N_{u^{\prime}}^{\prime} \geqq \max _{w \in L u^{\prime}} N_{w}-2+2 \alpha$. So, from (18), (22-24), we get (1) with primes.

Let us prove (4). We get, in the same way as we got (18),

$$
\min _{\boldsymbol{x}^{\prime} \in \mathbf{u}^{\prime}} \int_{\left(D^{\prime}\right) \mathbf{c}} d y^{\prime}\left(T^{\prime}+b^{\prime}\right)\left(x^{\prime}, y^{\prime}\right) \geqq \min _{x^{\prime} \in \mathbf{u}^{\prime}} b\left(x^{\prime}\right) c\left(u^{\prime},\left(D^{\prime}\right)^{c}\right),
$$

where $c\left(u^{\prime},\left(D^{\prime}\right)^{c}\right)$ is defined by replacing $L \hat{\mathbf{v}}^{\prime}$ in $(20)$ by $\left(D^{\prime}\right)^{c}$. We use (22) as before and

$$
c\left(u^{\prime},\left(D^{\prime}\right)^{c}\right) \geqq 1-\frac{2}{3} L^{-\frac{\alpha}{3}}
$$

which will be proven with the lemma. The proof of (4) is then similar to the one of (1) above.

Proof of Lemma. We have

$$
b\left(x^{\prime}\right) \geqq c_{1} \beta_{w} \sum_{t=1}^{\frac{L^{2}}{2}} \int_{i=1}^{t-1} \prod_{i=1}^{t-2} d \omega(i) \chi(\omega(i) \in \mathbf{w} \cap D) \prod_{i=0}^{t-2}(T+b)(\omega(i), \omega(i+1))
$$

with $\omega(0)=L x^{\prime}$, and where we used (1) and (17), which implies that $\tilde{\mathbf{w}} \nless D_{\ell}, \forall \tilde{w}$, $|w-\tilde{w}|=1$ since $L x^{\prime} \in \mathbf{w} \subset L \mathbf{u}^{\prime}$.

Now,

where

$$
(26) \geqq c_{1} \beta_{w} \frac{L^{2}}{2} \bar{b}\left(x^{\prime}\right),
$$

$$
\bar{b}\left(x^{\prime}\right)=\int \prod_{i=1}^{\frac{L^{2}}{2}} d \omega(i) \chi(\omega(i) \in \mathbf{w} \cap D) \prod_{i=0}^{\frac{L^{2}}{2}-1}(T+b)(\omega(i), \omega(i+1)) .
$$

Equation (27) holds because

$$
\int d y(T+b)(x, y)=1
$$

We shall prove that

$$
b\left(x^{\prime}\right)+\bar{b}\left(x^{\prime}\right) \geqq 1-e^{-c L^{\alpha}} .
$$


Equation (22) then follows from (26-29). To prove (29), we use (28):

$$
1=\int \prod_{i=1}^{\frac{L^{2}}{2}} d \omega(i)(\chi(\omega(i) \in \mathbf{w} \cap D)+\chi(\omega(i) \notin \mathbf{w} \cap D)) \prod_{i=0}^{\frac{L^{2}}{2}-1}(T+b)(\omega(i), \omega(i+1))
$$

with $\omega(0)=L x^{\prime}$,

$$
=\bar{b}\left(x^{\prime}\right)+\sum_{t=1}^{\frac{L^{2}}{2}} \int d y \chi(y \notin \mathbf{w} \cap D) \int d \omega_{L x^{\prime} y}^{t} \prod_{i=1}^{t-1} \chi(\omega(i) \in \mathbf{w} \cap D)(T+b)(\omega) .
$$

To get (31), we expand the product of characteristic functions in (30), we resum, and use (28). From (31) we get,

$$
1 \leqq \bar{b}\left(x^{\prime}\right)+b\left(x^{\prime}\right)+\frac{L^{2}}{2} \max _{x \in \mathbf{w} \cap D} \int d y(T+b)(x, y) \chi\left(|y-w| \geqq \frac{L^{\alpha}}{2}\right)
$$

which proves (29), using the exponential decay of $T$ and $b$. For $b$, this follows from (6.25) $-(6.27)$, the fact that $D_{\ell}$ is far from w because of (17) and the bound diam $\left(D\left(L x^{\prime}\right)\right) \leqq 1$ coming from $D\left(L x^{\prime}\right) \subset D_{s}$ (see Lemma 6.1).

We prove now (23). We write

$$
c\left(u^{\prime}, v^{\prime}\right) \geqq \min _{\substack{y \notin D \\|y-w| \leqq \frac{L^{\alpha}}{2}}}\left(\min _{t \leqq \frac{L^{2}}{2}} \int_{L \hat{\mathbf{v}}^{\prime}} d y^{\prime} T^{L^{2}-t}\left(y-y^{\prime}\right)-F_{y}\right)
$$

where

$$
F_{y}=\sum_{t=0}^{L^{2}} \int_{D} d \tilde{y} T^{t}(y-\tilde{y})+\int d \tilde{y} d z T^{t}(y-\tilde{y})(|s(\tilde{y}, z)|+|\varepsilon(\tilde{y}, z)|+|E(\tilde{y}, z)|) .
$$

This lower bound comes from first expanding

$$
\begin{gathered}
\int d \omega_{y y^{\prime}}^{L^{2}-t}(T+b)(\omega) \chi(\omega \cap D=\phi) \geqq \int d \omega_{y y^{\prime}}^{L^{2}-t} T^{L^{2}-t}(\omega) \chi(\omega \cap D=\phi) \\
-\sum_{t^{\prime}=0}^{L^{2}} \int d \tilde{y} T^{t^{\prime}}(y-\tilde{y})|b(\tilde{y}, z)| \chi(\tilde{y} \notin D),
\end{gathered}
$$

where we use (28) to bound the factors after $t^{\prime}$. Since $\tilde{y} \notin D$ we have no $\ell(\tilde{y}, z)$ in (34). We also expand $\chi(\omega \cap D=\phi)=\prod_{t^{\prime}=0}^{L^{2}-t}\left(1-\chi\left(\omega\left(t^{\prime}\right) \in D\right)\right)$

$$
\geqq 1-\sum_{t^{\prime}=0}^{L^{2}} \chi\left(\omega\left(t^{\prime}\right) \in D\right) \text {. }
$$

The first term in (35) gives the first term in (33) and the sum over $t^{\prime}$ gives the first term in (34).

To get a lower bound on (33), observe that

$$
\int_{L \hat{\mathbf{v}}^{\prime}} d y^{\prime} T^{L^{2}-t}\left(y-y^{\prime}\right) \geqq 3 c_{1}
$$

for some $c_{1}$, uniformly in $y \in L \mathbf{u}^{\prime}+B\left(\frac{L^{\alpha}}{2}\right), t \leqq \frac{L^{2}}{2}$; indeed $\left|u^{\prime}-v^{\prime}\right|=1$, and $\left|L \mathbf{v}^{\prime}\right|$ $\geqq L^{3} / 2$, since $\left|L \mathbf{v}^{\prime} \cap L D^{\prime}\right| \leqq c L^{\beta+3 \alpha}$, by (16) and (6.6).

We shall prove

$$
F_{y} \leqq \frac{1}{3} L^{-\frac{\alpha}{3}}
$$


for all $y \notin D,|y-w| \leqq \frac{L^{\alpha}}{2}$. This then proves (23). To prove (37), consider the first term in (34):

$$
\sum_{t} \int_{D} d \tilde{y} T^{t}(y-\tilde{y}) \chi\left(|\tilde{y}-y| \leqq \frac{L^{\alpha}}{2}\right) \leqq c \sum_{\left|v^{\prime}-u^{\prime}\right|<2}\left|D_{s} \cap L \mathbf{v}^{\prime}\right| \leqq c L^{-2}
$$

using (6.19) and (16), (17) $\left(|\tilde{y}-w| \leqq L^{\alpha}\right.$, hence, by (17), $\left.\tilde{y} \in D_{s}\right)$.

Moreover,

$$
\begin{aligned}
\sum_{t} \int_{D} d \tilde{y} T^{t}(y-\tilde{y}) \chi\left(\frac{L^{\alpha}}{2} \leqq|\tilde{y}-y| \leqq L^{1+\gamma}\right) & \leqq \sum_{z \notin D^{\prime}, t} \int_{|D \cap L \mathbf{z}|} d \tilde{y} T^{t}(y-\tilde{y}) \chi\left(\frac{L^{\alpha}}{2} \leqq|\tilde{y}-y|\right) \\
& \leqq c L^{\beta} L^{-\alpha} \sum_{z} e^{-\left|\frac{y}{L}-z\right|} \leqq c L^{\beta} L^{-\alpha}
\end{aligned}
$$

using (16), (A.22) and (6.17). Finally,

$$
\sum_{t} \int d y^{\prime} T^{t}\left(y-y^{\prime}\right) \chi\left(L^{1+\gamma} \leqq\left|y^{\prime}-y\right|\right) \leqq e^{-c L^{\gamma}}
$$

using (A.22). The $s$ and $E$ terms in (34) are easy to control, using (5.11), (6.26), and $L^{2}<\exp \left(c L^{\gamma}\right)$. For $\varepsilon(\tilde{y}, z)$, we use

$$
\int d \tilde{y} d z T^{t}(y-\tilde{y})|\varepsilon(\tilde{y}, z)| \leqq c \int d y^{\prime} T^{t}(y-\tilde{y}) e^{-d(\tilde{y}, D)} \sum_{Y} \int d z\left|\varepsilon_{Y}(\tilde{y}, z)\right| e^{d(\tilde{y} \cup z \cup Y)},
$$

since $\varepsilon_{Y}=0$ unless $N(Y) \neq-\infty$, i.e., using Lemma 6.1a, unless $d(Y, D)<2$. Then, by (6.25)

$$
\sum_{t}(41) \leqq c L^{-\alpha / 2} \sum_{t} \int d \tilde{y} T^{t}(y-\tilde{y}) e^{-d(\tilde{y}, D)} \leqq L^{-\alpha / 2} c L^{\beta}
$$

reasoning as in (38-40) and using (A.22), with $\mathbf{E}=\mathbf{D} \cap \mathbf{L u}$ for $u \notin \widetilde{D^{\prime}}$. Combining (38-42) proves (37).

We have only (25) left to prove. This is similar to the proof of (23). We have the bound (37) on $F_{y}$ and it is enough to show:

$$
\min _{y \in S_{w}} \min _{t \leqq L^{2} / 2} \int_{\left(L D^{\prime}\right)^{c}} d y^{\prime} T^{L^{2}-t}\left(y-y^{\prime}\right) \geqq 1-L^{-1}
$$

or

$$
\int_{L D^{\prime}} d y^{\prime} T^{L^{2}-t}\left(y-y^{\prime}\right) \leqq L^{-1}
$$

with $y, t$ as above. Divide the integral into $\left|y^{\prime}-y\right| \leqq \frac{L^{1+\gamma}}{2}$ and $\left|y-y^{\prime}\right|>\frac{L^{1+\gamma}}{2}$. The second part is negligible, by (A.22), and the first is bounded by

$$
c L^{-3} \sum_{v^{\prime} \notin D^{\prime} \cup R^{\prime}}\left|L D^{\prime} \cap L \mathbf{v}^{\prime}\right| e^{-\left|\frac{y}{L}-v^{\prime}\right|} \leqq c L^{-2},
$$

using $t \leqq \frac{L^{2}}{2}$ to get $c L^{-3}\left[\right.$ see (A.20)], and (6.18) to bound $\left|L D^{\prime} \cap L \mathbf{v}^{\prime}\right|=L^{3}\left|D^{\prime} \cap \mathbf{v}^{\prime}\right|$ $\leqq L^{3} L^{-2}$ since $\mathbf{v}^{\prime} \in D_{\ell}^{\prime}$, by (16).

\section{Probabilistic Estimates}

We shall show that the probability that $N_{n u} \neq-\infty$ is bounded by $\exp (-\mathcal{O}(n))$. This will be used in Sect. 10. It also implies (5.50), as we show below. 
Proposition 1. For any sets $A, B \subset \mathbf{Z}^{d}$, any numbers

$$
\left\{N_{u} \in(1-2 \alpha) \mathbf{Z}, N_{u}>-2 n\left(1-2 \alpha+\frac{2 \alpha}{2+\alpha}\right)\right\}_{u \in A}
$$

and for all $n \geqq n_{0}$,

$$
\begin{aligned}
& \mathscr{P}\left(\left\{N_{n u}=N_{u}\right\}_{u \in A},\left\{q_{v}=1\right\}_{v \in B}\right) \\
& \quad \leqq \exp \left[-\hat{\Gamma}\left((2-2 \alpha-\beta) n\left(|A|+L^{\gamma}|B|\right)-\left(1+n^{-1}\right) \sum_{u \in A} N_{u}\right)\right]
\end{aligned}
$$

where $\mathscr{P}$ is the probability defined on the set of $\left\{b(x, y), x, y \in \mathbf{Z}^{d}\right\}, \hat{\Gamma}=\Gamma^{1 / 2}$, and $q_{v}=\chi\left(v \in D_{\ell}\right)$.

If one forgets about $q_{v}$, the flow is as explained in Sect. 3, up to $-\beta$, and can be understood on the basis of (6.10-6.14). We introduce $q_{v}$ because it enters in the definition of $N$, see $(6.10,6.8)$. The bound (1) remembers that $D_{\ell}$, i.e. $q_{v}=1$ is unlikely. We shall also have a contribution from $R$, for which we use the results of Sect. 5. To control the occurrence of several $N^{\prime} s$ in a box, we shall use the fact that we have a small factor, in the right-hand side of (1), for each site in $A$. We have also to control negative $N^{\prime} s$. Here, we use the fact that large fields are reabsorbed long before they are likely to meet, which is reflected in the lower bound on allowed values of $N$. This bound implies that negative $N^{\prime} s$ are controlled by the small factor in (1), see $(14,15)$ below.

First, we show how to get (5.50) from Proposition 1. Since $r=e+\ell$, we get, from $(6.25-6.27)$,

$$
\int d y\left|r_{n}(x, y)\right| \leqq c \sum_{Y \subset N} \exp \left[-d(x \cup y \cup Y)+c \sum_{u \in Y}^{+} N_{u}\right]
$$

Here, we use $c \sum_{u \in Y \cap D_{\ell}}^{+} N_{u} \geqq d\left(Y \cap D_{\ell}\right)$ since $D_{\ell} \in D_{+}$. Then, (5.50) follows easily, for $\Gamma \gg L^{4}$, from $\mathscr{P}(Y \subset N) \leqq \exp (-c n \Gamma|Y|)$, which itself is a consequence of (1) [see e.g. inequality (14) below].

Proof of Proposition 1. We proceed by induction: For $n=n_{0}$, (1) follows from our assumption (2.12) on the probability of large $b(x, y)$, since we take $\Gamma=L n_{0}^{2}$. Define $m_{n v}=\chi\left(v \in R_{n}+B\left(3 L^{\gamma}\right)\right)$. Then, it is clear that $\left\{N_{n+1 u^{\prime}}\right\}_{u^{\prime} \in A^{\prime}},\left\{q_{n+1 v^{\prime}}\right\}_{v^{\prime} \in B^{\prime}}$ are determined through $(6.5,6.10-6.14)$ by

$$
\left\{N_{n u}, q_{n v}, u, v \in L\left(\left(A^{\prime} \cup B^{\prime}\right)+B\left(3 L^{\gamma}\right)\right\}\right)
$$

and by $\left\{m_{n+1 v^{\prime}}, v^{\prime} \in A^{\prime} \cup B^{\prime}\right\}$. So, we may write

$$
\begin{aligned}
& \mathscr{P}\left(\left\{N_{n+1 u^{\prime}}=N_{u}^{\prime}\right\}_{u^{\prime} \in A^{\prime}},\left\{q_{n+1 v^{\prime}}\right\}_{v^{\prime} \in B^{\prime}}\right) \\
& =\sum^{*} \hat{P}\left(\left\{N_{n u}=N_{u}\right\}_{u \in A},\left\{q_{n v}=1\right\}_{v \in B},\left\{m_{n+1 v^{\prime}}=1\right\}_{v^{\prime} \in C^{\prime}}\right),
\end{aligned}
$$

where $\hat{P}$ is the probability that the indicated events occur and that $N_{n u}=-\infty$ for $u \in L\left(\left(A^{\prime} \cup B^{\prime}\right)+B\left(3 L^{\gamma}\right)\right) \backslash A, q_{n v}=0$ for $v \in L\left(\left(A^{\prime} \cup B^{\prime}\right)+B\left(3 L^{\gamma}\right)\right) \backslash B$ and $m_{n u^{\prime}}=0$ for $u^{\prime} \in\left(A^{\prime} \cup B^{\prime}\right) \backslash C^{\prime}$. The sum runs over all sets $A, B$, and $C^{\prime}$ such that

$$
\begin{gathered}
A, B \subset L\left(\left(A^{\prime} \cup B^{\prime}\right)+B\left(3 L^{\gamma}\right)\right), \\
C^{\prime} \subset A^{\prime} \cup B^{\prime}
\end{gathered}
$$

and over all values of $N_{u} \in(1-2 \alpha) Z, u \in A$ such that $\left\{N_{n+1 u^{\prime}}, q_{n+1, v^{\prime}}\right\}$, determined by $(6.5,6.10-6.14)$, take the values given in the left-hand side of $(2)$. Note that the 
values of $n_{u^{\prime}}$, for $u^{\prime} \in A^{\prime} \cup B^{\prime}$, are also determined, for each term in $\sum^{*}$, see (6.8).

For each $u^{\prime} \in A^{\prime} \cup B^{\prime}$, there must be a $u \in L\left(\mathbf{u}^{\prime}+B\left(3 L^{\gamma}\right)\right)$ with $N_{n u} \neq-\infty$ or $q_{n u}=1$ or $m_{n+1 u^{\prime}}=1$. Therefore, for each term in (2),

$$
\left|\left[L^{-1} A\right]\right|+\left|\left[L^{-1} B\right]\right|+\left|C^{\prime}\right| \geqq c L^{-3 \gamma}\left(\left|A^{\prime}\right|+\left|B^{\prime}\right|\right) .
$$

Now we want to bound (2) by the right-hand side of (1) with primes and $n \rightarrow n+1$. First, we have an upper bound on (2) if we replace $\hat{P}$ by $\mathscr{P}$, i.e. if we do not specify the values of $N, q, m$ outside $A, B, C^{\prime}$, but still keeping the constraints on the sum, so that, in particular, (5) holds.

The next step is to use Hölder's inequality to separate the estimates on $N, q$ and on $m$ :

$$
\begin{gathered}
\mathscr{P}\left(\left\{N_{n u}=N_{u}\right\}_{u \in A},\left\{q_{n v}=1\right\}_{v \in B},\left\{m_{n+1 u^{\prime}}\right\}_{u^{\prime} \in C^{\prime}}\right) \\
\leqq \mathscr{P}\left(\left\{N_{n u}=N_{u}\right\}_{u \in A},\left\{q_{n v}=1\right\}_{v \in B}\right)^{1-n^{-3} \mathscr{P}\left(\left\{m_{n+1 u^{\prime}}\right\}_{u^{\prime} \in C^{\prime}}\right)^{n^{-3}} .}
\end{gathered}
$$

To bound the last factor, we use the results of Sect. 5: (5.12) and (5.15) imply, using Tchebychev's inequality, and the fact that one has at most $c L^{6 n}$ values of $\xi$, that the probability that $u \in R_{n}[\operatorname{see}(5.90,5.64)]$ is less than

$$
\exp \left[\left(-c \delta_{n}^{2} / \varepsilon_{n}^{2}\right)=\exp \left(-c L^{\alpha n}\right)\right.
$$

using (3.54) and (3.66). So,

$$
\mathscr{P}\left(\left\{m_{n+1 u^{\prime}}\right\}_{u^{\prime} \in C^{\prime}}\right)^{n^{-3}} \leqq \exp \left(-L^{y n}\left|C^{\prime}\right|\right)
$$

follows, using Schwartz' inequality a few times and the independence of $s_{n u}, s_{n v}$ for $|u-v| \geqq 2$.

Inserting (7) and (1) in (6) and then (6) in (2), we have

$$
\begin{aligned}
& \mathscr{P}\left(\left\{N_{n+1 u^{\prime}}=N_{u^{\prime}}^{\prime}\right\}_{u^{\prime} \in A^{\prime}},\left\{q_{n+1 v^{\prime}}=1\right\}_{v^{\prime} \in B^{\prime}}\right) \\
& \quad \leqq \sum^{*} \exp \left[-L^{\gamma n}\left|C^{\prime}\right|-\Gamma_{n}(2-2 \alpha-\beta) n\left(|A|+L^{\gamma}|B|\right)-\Gamma_{n}\left(1+n^{-1}\right) \sum_{u \in A} N_{u}\right],
\end{aligned}
$$

where $\Gamma_{n}=\hat{\Gamma}\left(1-n^{-3}\right)$.

We have to prove that

$$
\begin{aligned}
& \sum^{*} \exp \left\{-L^{\gamma n}\left|C^{\prime}\right|-\Gamma_{n}(2-2 \alpha-\beta) n\left(|A|+L^{\gamma}|B|\right)\right. \\
& \quad+\Gamma(2-2 \alpha-\beta)(n+1)\left(\left|A^{\prime}\right|+L^{\gamma}\left|B^{\prime}\right|\right) \\
& \left.\quad-\Gamma_{n}\left(1+n^{-1}\right) \sum_{u \in A} N_{u}+\Gamma\left(1+(n+1)^{-1}\right) \sum_{u^{\prime} \in A^{\prime}} N_{u^{\prime}}^{\prime}\right\} \leqq 1 .
\end{aligned}
$$

We shall first simplify the argument of the exponential with the following

\section{Lemma.}

a)

$$
\left|A^{\prime}\right| \leqq\left|\left[L^{-1} A\right]\right|+\sum_{u^{\prime} \in A^{\prime}} n_{u^{\prime}}
$$

b) $\quad \Gamma(2-2 \alpha-\beta)(n+1) L^{\gamma}\left|B^{\prime}\right| \leqq 2 L^{\gamma} \Gamma n\left|C^{\prime}\right|+\Gamma_{n} n \gamma \sum_{u^{\prime}:\left|A \cap L \mathbf{u}^{\prime}\right| \geqq 2}\left(\left|A \cap L \mathbf{u}^{\prime}\right|-1\right)$,

c) $\quad \Gamma\left(1+(n+1)^{-1}\right) \sum_{u^{\prime} \in A^{\prime}} N_{u^{\prime}}^{\prime} \leqq \Gamma_{n}\left(1+n^{-1}\right) \sum_{u \in A} N_{u}-\Gamma\left(1+n^{-1}\right) \sum_{u \in A}^{-} N_{u}$

$$
-\Gamma\left(2 n^{2}\right)^{-1} \sum_{u \in A}^{+} N_{u}+2 \Gamma c_{0} \sum_{u^{\prime} \in A^{\prime}}^{+} n_{u^{\prime}}-\Gamma(2-2 \alpha)\left|A^{\prime}\right|,
$$

d)

$$
\sum_{u^{\prime} \in A^{\prime} \cup B^{\prime}} n_{u^{\prime}} \leqq 2\left|C^{\prime}\right|+\gamma \sum_{u^{\prime}:\left|A \cap L \mathbf{u}^{\prime}\right| \geqq 2}\left(\left|A \cap L \mathbf{u}^{\prime}\right|-1\right)+\gamma|B| .
$$


Using this lemma, we bound the left-hand side of (9) by

$$
\begin{aligned}
& \sum^{*} \exp \left[-L^{y n / 2}\left|C^{\prime}\right|-\frac{\beta}{2} \Gamma\left|\left[L^{-1} A\right]\right|-3 n \Gamma\right. \\
& \quad \times \sum_{u^{\prime} \in A^{\prime} \cup B^{\prime}} n_{u^{\prime}}-\Gamma_{n}(2-2 \alpha-2 \beta) n \sum_{\left|A \cap L \mathbf{u}^{\prime}\right| \geqq 2}\left(\left|A \cap L \mathbf{u}^{\prime}\right|-1\right) \\
& \left.\quad-\Gamma\left(1+n^{-1}\right) \sum_{u \in A}^{-} N_{u}-\Gamma\left(2 n^{2}\right)^{-1} \sum_{u \in A}^{+} N_{u}-\Gamma n L^{\gamma}|B|\right]
\end{aligned}
$$

To get that, we inserted (12) in (10) and in (11), for the term $\sum n_{u^{\prime}}$. We used the easy bounds:

$$
\begin{gathered}
-L^{\gamma n}+2 L^{\gamma} \Gamma n+2 \Gamma(2-2 \alpha-\beta)(n+1)+6 n \Gamma+4 \Gamma c_{0} \leqq-L^{y n / 2} \\
\text { for the }\left|c^{\prime}\right| \text { term }
\end{gathered}
$$

(remember that $\Gamma=L n_{0}^{2}$, so that $L^{y n}$ dominates)

$$
-\Gamma_{n}(2-2 \alpha-\beta) n+\Gamma(2-2 \alpha-\beta)(n+1)-\Gamma(2-2 \alpha) \leqq-\frac{\beta}{2} \Gamma,
$$

and $|A|=\left|\left[L^{-1} A\right]\right|+\sum_{\left|A \cap L \mathbf{u}^{\prime}\right| \geqq 2}\left(\left|A \cap L \mathbf{u}^{\prime}\right|-1\right)$ for the $\left|\left[L^{-1} A\right]\right|$ term.

$$
\begin{aligned}
& -\Gamma_{n}(2-2 \alpha-\beta) n+\Gamma_{n} n \gamma+2 \Gamma c_{0} \gamma+\Gamma(2-2 \alpha-\beta)(n+1) \gamma+3 \gamma n \Gamma \\
& \quad \leqq-\Gamma_{n}(2-2 \alpha-2 \beta) n
\end{aligned}
$$

for $\left|A \cap L \mathbf{u}^{\prime}\right|$. Here we use $\gamma \ll \beta$.

Finally,

$$
-\Gamma_{n}(2-2 \alpha-\beta) n L^{\gamma}+\Gamma(2-2 \alpha-\beta)(n+1) \gamma+2 \Gamma c_{0} \gamma+3 n \gamma \Gamma \leqq-\Gamma n L^{\gamma}
$$

for the $|B|$ term.

To show that (13) is bounded by 1 , consider first the sum over $\left\{N_{u}\right\}_{u \in L v^{\prime}}$, for each $v^{\prime} \in\left[L^{-1} A\right]$. For any fixed $u$, we use

$$
\begin{aligned}
& \sum_{N_{u}>-n(2-2 \alpha-3 \beta)}^{-} \exp \left(-\Gamma\left(1+n^{-1}\right) N_{u}\right)+\sum_{N_{u}}^{+} \exp \left(-\Gamma\left(2 n^{2}\right)^{-1} N_{u}\right) \\
& \leqq\left(e^{\Gamma(n(2-2 \alpha-3 \beta)+2)}+\frac{n^{2}}{\Gamma}\right),
\end{aligned}
$$

where the constraint $N_{u}>-n(2-2 \alpha-3 \beta)$ comes from (6.14) and $\beta \ll \alpha^{2}$. For the sum in $L \mathbf{v}^{\prime}$, let $p_{v^{\prime}}=\left|A \cap L \mathbf{v}^{\prime}\right|$. If $p_{v^{\prime}}=1$, then $N_{u}$ is determined, via (6.10-6.14) by the values of $N_{v^{\prime}}^{\prime}, n_{v^{\prime}}$, so there is no sum over $N_{u}$ in that case. However, there are $L^{3}=\left|L \mathbf{v}^{\prime}\right|$ possibilities for the single $u$, with $N_{u} \neq-\infty$, in $L \mathbf{v}^{\prime}$. Also, if $p_{v^{\prime}} \geqq 2$ but $N\left(L \mathbf{v}^{\prime}\right)<0$, the largest value of $N_{u}$, for $u \in L \mathbf{v}^{\prime}$, is determined, via (6.10-6.14), by $N_{v^{\prime}}^{\prime}$, if $n_{v^{\prime}}=0$. So, if $n_{v^{\prime}}=0$,

$$
\begin{gathered}
\sum_{A \subset L \mathbf{v}^{\prime}} \sum_{\left\{N_{u}\right\}_{u \in A}} \exp \left[-\Gamma\left(1+n^{-1}\right) \sum_{u \in A}^{-} N_{u}-\Gamma\left(2 n^{2}\right)^{-1} \sum_{u \in A}^{+} N_{u}\right. \\
\left.\Gamma_{n}(2-2 \alpha-2 \beta) n\left(p_{v^{\prime}}-1\right)\right] \\
\left.\leqq L^{3}+\sum_{p \geqq 2}\left(\begin{array}{c}
L^{3} \\
p
\end{array}\right)\left(c(\exp (\Gamma((2-2 \alpha-3 \beta) n)+2))+\frac{n^{2}}{\Gamma}\right)\right)^{p-1} \\
\quad \times \frac{c n^{2}}{\Gamma} \exp \left(-\Gamma_{n}(2-2 \alpha-2 \beta) n(p-1)\right) \leqq c L^{3} .
\end{gathered}
$$


In the first inequality, we used (14) for the $p-1$ sums over $N_{u}$, where $N_{u}$ may be negative; $\frac{c n^{2}}{\Gamma}$ bounds the remaining sum over $N_{u} \geqq 0 ;\left(\begin{array}{c}L^{3} \\ p\end{array}\right)$ counts the possible locations of $u$ 's in $L \mathbf{v}^{\prime}$ with $N_{u} \neq-\infty$. The second inequality follows because the sum over $p$ is controlled by $\exp (-\beta \Gamma n(p-1))$, which is the leading term.

If $n_{v^{\prime}}=1$, the only change is that we have a sum over $p N_{u}$ 's instead of $p-1$ and the left-hand side of (15) is bounded by

$$
c L^{3} \exp \left(n_{n^{\prime}}, \Gamma 2 n\right) .
$$

Using this bound to control, in (13), the sum over $\left\{N_{u}\right\}$ and over $A$ in each $L \mathbf{v}^{\prime}$ for $v^{\prime} \in\left[L^{-1} A\right]$, we have

$$
(13) \leqq \sum_{\hat{A}, B, C^{\prime}} \exp \left[-L^{y n / 2}\left|C^{\prime}\right|-\frac{\beta}{2} \Gamma|\hat{A}|-\Gamma n L^{\gamma}|B|\right]\left(c L^{3}\right)^{|\hat{A}|},
$$

where $\sum^{\prime}$ runs over

$$
\left.\hat{A} \subset\left(A^{\prime} \cup B^{\prime}\right)+B\left(3 L^{\gamma}\right)\right)
$$

and over $B, C^{\prime}$ as before, such that

$$
|\hat{A}|+\left|\left[L^{-1} B\right]\right|+\left|C^{\prime}\right| \geqq c L^{-3 \gamma}\left(\left|A^{\prime}\right|+\left|B^{\prime}\right|\right) .
$$

$\hat{A}=\left[\frac{A}{L}\right]$ before, since we have summed over $A$ in $L \mathbf{v}^{\prime}$. The $n_{v^{\prime}}$ in (16) is controlled by the corresponding term in (13); (19) is simply a rewriting of (5). To bound (17) by 1 is easy:

Using (19), each term of the sum is less than $\exp \left(-\Gamma L^{-4 \gamma}\left(\left|A^{\prime}\right|+\left|B^{\prime}\right|\right)\right)$, so we can take out of the sum the square root of each term. The remaining sum is bounded by

$$
\exp \left(\mathcal{O}(1)\left(\left|A^{\prime}\right|+\left|B^{\prime}\right|\right)\right)
$$

using $(3,4,18)$ and $\left(1+e^{\left.-\frac{\beta}{4} \Gamma\right)^{c L^{3(1+\gamma)}}}<c\right.$.

This finishes the proof of the proposition, so we are left with the

Proof of Lemma 1. a) If $N_{n+1 u^{\prime}} \neq-\infty$, then either $N\left(L u^{\prime}\right) \neq-\infty$, i.e. $u^{\prime} \in\left[L^{-1} A\right]$ or $n_{u^{\prime}}=1$ (or both).

b)

$$
\left|B^{\prime}\right|=\Sigma_{u^{\prime}} q_{u^{\prime}}^{\prime} \leqq \Sigma_{u^{\prime}}\left(m_{u^{\prime}}^{\prime}+L^{-\beta} \sum_{\substack{\left|A \cap \mathbf{v}^{\prime}\right| \geqq L^{\beta} \\\left|v^{\prime}-u^{\prime}\right| \geqq 2 L^{\gamma}}}\left|A \cap L \mathbf{v}^{\prime}\right|\right)
$$

because if $u^{\prime} \in D_{\ell}^{\prime}$, then either $u^{\prime} \in R^{\prime}+B\left(2 L^{\gamma}\right)$, i.e. $m_{u^{\prime}}^{\prime}=1$ or there exists a $v^{\prime}$, with $\left|v^{\prime}-u^{\prime}\right| \leqq 2 L^{\gamma}$ and $\left|A \cap L \mathbf{v}^{\prime}\right| \geqq L^{\beta} \quad$ [see (6.5)]. Using $\sum_{\left|v^{\prime}-u^{\prime}\right| \leqq 2 L^{\gamma}} 1 \leqq c L^{3 \gamma}$ and $c L^{3 \gamma} L^{-\beta} \ll \gamma$, we obtain b).

c) Consider first $u^{\prime}$ such that $N\left(L \mathbf{u}^{\prime}\right)=0$.

Then using (6.10-6.14) and

$$
1+(n+1)^{-1} \leqq\left(1+n^{-1}\right)\left(1-n^{-3}\right)-\left(2 n^{2}\right)^{-1}
$$

for $n \geqq n_{0}$, we get from (6.10),

$$
\begin{aligned}
\Gamma\left(1+(n+1)^{-1}\right) N_{u^{\prime}}^{\prime} \leqq & \Gamma_{n}\left(1+n^{-1}\right) \sum_{u \in L \mathbf{u}^{\prime} \cap A}^{+} N_{u}-\Gamma\left(2 n^{2}\right)^{-1} \\
& \times \sum_{u \in L \mathbf{u}^{\prime} \cap A}^{+} N_{u}+2 \Gamma c_{0} n_{u^{\prime}}-\Gamma(2-2 \alpha) .
\end{aligned}
$$


To get (11), we use also

$$
\Sigma^{+} N_{u}=\Sigma N_{u}-\Sigma^{-} N_{u} .
$$

Now let $N\left(L \mathbf{u}^{\prime}\right)<0$, then

$$
N_{u}^{\prime} \leqq c_{0} n_{u^{\prime}}-(2-2 \alpha) .
$$

Combining (20), (21), and (22), gives (11).

d) This is similar to b) above; $n_{u^{\prime}}=1$ means

$$
u^{\prime} \in D_{\ell}^{\prime}+B\left(L^{\gamma}\right) \quad \text { or } \quad L \mathbf{u}^{\prime} \cap\left(D_{\ell}+B\left(L^{\alpha}\right)\right) \neq \phi .
$$

$u^{\prime} \in D_{\ell}^{\prime}+B\left(L^{\gamma}\right)$ is controlled by the first two terms in (12), as in the proof of b). If $L \mathbf{u}^{\prime} \cap\left(D_{\ell}+B\left(L^{\alpha}\right)\right) \neq \phi$, then there must exist a $v^{\prime}$, with $\left|v^{\prime}-u^{\prime}\right|<2$ and $\left|D_{\ell} \cap L \mathbf{v}^{\prime}\right|$ $\geqq c L^{3 \gamma}$, because each connected component of $D_{\ell}$ contains at least $\mathcal{O}\left(L^{3 \gamma}\right)$ unit blocks, by construction. So,

$$
\chi\left(L \mathbf{u}^{\prime} \cap\left(D_{\ell}+B\left(L^{\alpha}\right)\right) \neq \emptyset\right) \leqq c L^{-3 \gamma} \sum_{\left|v^{\prime}-u^{\prime}\right|<2}\left|D_{\ell} \cap L \mathbf{v}^{\prime}\right|,
$$

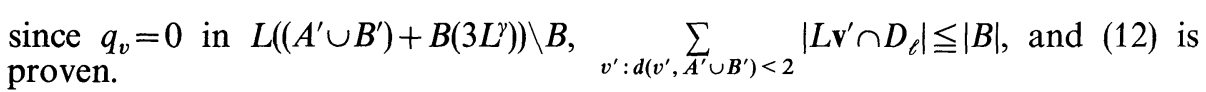

\section{Proof of Theorems 1 and 2}

First we define the set of measure one on which the convergence holds:

$$
\mathscr{B}=\left\{\mathbf{b}|\exists m(\mathbf{b})| \text { such that } N_{n u}=-\infty, \forall n \geqq m(\mathbf{b}), \forall u,|u| \leqq L^{n}\right\} .
$$

The reason for introducing $\mathscr{B}$ is that it contains environments such that $b_{n}(x, y)$ $=s_{n}(x, y)$ for all $|x|,|y| \leqq L^{n}$ and $n \geqq m(\mathbf{b})$. This follows from Proposition 6.1 and Lemma 6.1 a: $e$ and $\ell$ are zero away from $D$, and $D$ is empty if $N_{n u}=-\infty$. Besides, note that in time $T \leqq L^{2 n}$ the walk cannot move at a (rescaled) distance larger than $L^{n}[$ see (3.26)] so, for b in (1), the walk only "sees" small fields for all times larger than $L^{2 m(\mathbf{b})}$. For small fields, we may use the deterministic bounds $(5.10,5.11,5.14)$.

Lemma. $\mathscr{P}(\mathscr{B})=1$.

Proof. This follows directly from Proposition 9.1. The latter implies

$$
\mathscr{P}\left(N_{n u} \neq-\infty\right) \leqq \exp \left(-\Gamma \frac{\beta}{2} n\right)
$$

by summing (9.1) over $N_{u} \geqq-2 n\left(1-2 \alpha+\frac{2 \alpha}{2+\alpha}\right)$, and using $\beta \ll \alpha^{2}$. Thus,

$$
\mathscr{P}\left(\exists u,|u| \leqq L^{n}, N_{n u}=-\infty\right) \leqq c L^{3 n} \exp \left(-\Gamma \frac{\beta}{2} n\right),
$$

and the lemma follows, for $\Gamma$ large enough.

Proof of Theorem 1. Since $f_{i}$ and the paths $\omega$ are continuous, it is enough to prove Theorem 1 for $t_{i}$ in the dense set $\cup L^{-2 l} \mathbf{N}$. We have, for $t_{i} \in L^{-2 l} \mathbf{N}, \forall i=1, \ldots, k$ and for $n \geqq l$,

$$
\int d v_{n}(\omega) \prod_{i} f_{i}\left(\omega\left(t_{i}\right)\right)=\int d y \int d \omega_{0 y}^{L^{2 l}}\left(T_{m}+b_{m}\right)(\omega) \prod_{i} f\left(L^{-1} \omega\left(L^{2 l} t_{i}\right)\right)
$$


with $m=n_{0}+n-l$ (in Sect. 2, we started with $n=0$, while in the right-hand side of (2), we start with $n=n_{0}$ ). Let now $\mathbf{b} \in \mathscr{B}$. Thus, for $m$ large enough, $b_{m}=s_{m}$. Write

$$
T_{m}+s_{m}=T_{D}^{*}+\tilde{s}_{m}
$$

with $T_{D}^{*}$ given by (3.22). We see that the right-hand side (2) consists of a fixed time $\left(=L^{2 l}\right)$ problem with $\tilde{s}_{m}$ tending to zero as $n \rightarrow \infty$. Now,

$$
\int d v^{D}(\omega) \prod_{i} f\left(\omega\left(t_{i}\right)\right)=\int d y \int d \omega_{0 y}^{L^{2 l}} T_{D}^{*}(\omega) \prod_{i} f\left(L^{-1} \omega\left(L^{2 l} t_{i}\right)\right) .
$$

Moreover, for any $f \in \mathscr{F}$, an integration by parts gives

and, we have

$$
f(\xi) \leqq c \frac{\exp \left(+|u|^{1 / 2}\right)}{|k|}
$$

$$
\sum_{\xi_{2}}\left|\tilde{s}_{m}\left(\xi_{1}, \xi_{2}\right) f\left(\xi_{2}\right)\right| e^{p\left|u_{2}\right|^{1 / 2}} \leqq c(f) \exp \left[(p+1)\left|u_{1}\right|^{1 / 2}+c(p+1)^{2}\right] L^{-m / 4} .
$$

For (4), we used Theorem 5.1 for $T_{m}-T_{D}^{*}$, (5.14) for $s_{m}$, and the bound

$$
\sum_{u_{2} \in \mathbf{Z}^{d}} e^{-\left|u_{1}-u_{2}\right|} e^{(p+1)\left|u_{2}\right|^{1 / 2}} \leqq e^{\left((p+1)\left|u_{1}\right|^{1 / 2}+c(p+1)^{2}\right)} .
$$

We get, expanding $\tilde{s}_{m}$ in (2), and using (5.27) and (4),

$$
\left|\int d v_{n} \prod f_{i}-\int d v^{D} \prod f_{i}\right| \leqq c\left(k, f_{i}\right) L^{-a n}
$$

for some $a>0$. The claim follows.

Proof of Theorem 2. This follows from Theorem 1 and the fact that the sequence $\left(v_{T}\right)$ is tight. To prove tightness, we use a moment condition (see e.g. [16, Theorem 12.3]): We show that $\exists \alpha>0$, such that $\forall \mathbf{b} \in \mathscr{B}, \exists K$, such that $\forall t, s \in[0,1]$,

$$
\int d v_{T}(\omega)(\omega(s)-\omega(t))^{4} \leqq K|s-t|^{1+\alpha} .
$$

Since $|\omega(s)-\omega(t)| \leqq T^{1 / 2}|s-t|\left[\right.$ see (2.4)] for $[s T]=[t T]$, i.e. for $|s-t| \leqq T^{-1},(5)$ is trivial in that case. For $[s T] \neq[t T]$, we rewrite (5) in terms of lattice walks. For those, it is enough to show:

$$
\int d \mu_{T}(\omega)(|\omega(i)-\omega(j)|+2)^{4} \leqq K T^{1-\alpha}|i-j|^{1+\alpha},
$$

where $i=[t T], j=[t T] \in N,|i-j| \neq 0$, and $\omega$ are now lattice walks.

We write

$$
i=\sum_{k=0}^{n} i_{k} L^{2 k}, \quad j=\sum_{k=0}^{n} j_{k} L^{2 k}
$$

with $0 \leqq i_{k}, j_{k} \leqq L^{2}-1$, and $n=\max \left\{k: L^{2 k} \leqq T\right\}$. Let

$$
i^{\prime}=\sum_{k=m}^{n} i_{k} L^{2 k}, \quad j^{\prime}=\sum_{k=m}^{n} j_{k} L^{2 k}
$$

where $m=m(\mathbf{b})=\min \left\{m\left|N_{n u}=-\infty \quad \forall n \geqq m \quad \forall\right| u \mid \leqq L^{n}\right\}$.

Using $|\omega(i)-\omega(i+1)|=1$, we have

$$
|\omega(i)-\omega(j)| \leqq\left|\omega\left(i^{\prime}\right)-\omega\left(j^{\prime}\right)\right|+2 L^{2 m} .
$$

Inserting (9) in the left-hand side of (6), we get

$$
\int d \mu_{T}(\omega)(|\omega(i)-\omega(j)|+2)^{4} \leqq c(m)\left(1+\int d \mu_{T}(\omega)\left(\omega\left(i^{\prime}\right)-\omega\left(j^{\prime}\right)\right)^{4}\right),
$$

where $c(m)$ is a constant dependent on $m(\mathbf{b})$, i.e. on $\mathbf{b}$. 
Now, let $j^{\prime}>i^{\prime}$;

$$
\begin{gathered}
\int d \mu_{T}(\omega)\left(\omega\left(i^{\prime}\right)-\omega\left(j^{\prime}\right)\right)^{4}=\int \prod_{k=m}^{n+1} d x_{k} \int d \omega_{0 x_{m}}^{i^{\prime}}(T+b)(\omega) \\
\times \prod_{k=m}^{n}\left(T_{k}+s_{k}\right)^{\tau_{k}}\left(x_{k}, x_{k+1}\right)\left(\sum_{k=m}^{n} L^{k}\left(x_{k+1}-x_{k}\right)\right)^{4},
\end{gathered}
$$

where we use $b_{k}=s_{k}$ for $k \geqq m$, and write $j^{\prime}-i^{\prime}=\sum_{k=m}^{n} \tau_{k} L^{2 k}$. $p \leqq 4$,

Now, use (3), and expand the product over $T_{D}^{*}+S_{k}$ and the $\left(\sum_{k}\right)^{4}:$ obviously, for
,

$$
\int\left(T_{D}^{*}\right)^{\tau}(x-y)(x-y)^{p} \leqq c \tau^{\frac{p}{2}}
$$

Moreover, this integral vanishes if $p$ is odd, or if $\tau=0$. We get, using (4),

The net result is

$$
\left|\int \tilde{s}_{k}(x, y)(x-y)^{p} d y\right| \leqq c L^{-\frac{k}{4}}
$$

From (7), (8),

$$
(11) \leqq c\left(\sum_{k=m}^{n} \tau_{k} L^{2 k}\right)^{2} \leqq c\left|i^{\prime}-j^{\prime}\right|^{2} .
$$

$$
\left|i^{\prime}-j^{\prime}\right|^{2} \leqq\left(|i-j|+2 L^{2 m}\right)^{2} \leqq K(m)|i-j|^{1+\alpha} T^{1-\alpha}
$$

using $|i-j| \neq 0$.

Combining (10), (11), (13), (14), we get (6), i.e. (5), and tightness holds.

\section{Appendix}

We prove Lemmas 1 and 3-8 entering the proof of the upper bounds in Sect. 6.

Proof of Lemma 1. a) The proof of (6.15) is by induction. For $n=n_{0}, D=N$ [see $(3.76-3.77)]$. Assume that $\mathbf{u} \cap D^{\prime} \neq \emptyset$. We shall prove that there must be a $v$, with $|v-u|<2$ and $N_{v}^{\prime} \neq-\infty$. If $n_{u}=1$, then $N_{u}^{\prime} \neq-\infty$ follows from (6.10-6.14). Assume $n_{u}=0$, so $\mathbf{u} \cap D_{\ell}^{\prime}=\phi$. If $L \mathbf{u} \cap\left(D_{+}+B\left(L^{\alpha}\right)\right) \neq \phi$, then clearly, using (6.15) inductively, $N_{+} \cap L \mathbf{v}$ must be non-empty for some $v$, with $|v-u|<2$ and $N_{v}^{\prime} \neq-\infty$. Now, if $\operatorname{Lu} \cap\left(\mathbf{D}_{-} \backslash L \mathscr{D}\right) \neq \phi$, we must have $N(L u) \neq-\infty$ otherwise, $\tilde{N}_{u}=-\infty$ (because $\left.n_{u}=0\right)$ and $\mathbf{u} \in \mathscr{D}$. But, $N(L u) \neq-\infty$ imply $N_{u}^{\prime} \neq-\infty$ unless, again, if $\mathbf{u} \in \mathscr{D}$. So, $\mathbf{u} \cap D_{\ell}^{\prime}=\phi, L \mathbf{u} \cap\left(D_{+}+B\left(L^{\alpha}\right)\right)=\phi$ and $L \mathbf{u} \cap\left(\mathbf{D}_{-} \backslash L \mathscr{D}\right)=\phi$. From (6.6), we get $\mathbf{u} \cap D^{\prime}=\phi$.

Equation (6.16) is proven inductively, using (6.10), (6.11), where all terms are positive, when $n_{u}=1$, since we chose $c_{0}>2-2 \alpha$.

b) Equation (6.17) follows from (6.15) and the definition of $\widetilde{D}^{\prime}$.

c) For (6.18), we proceed inductively, with $D_{s}=\phi$ when $n=n_{0}$. Assume $\mathbf{v} \measuredangle D_{\ell}^{\prime}$, otherwise $D_{s}^{\prime} \cap \mathbf{v}=\phi$. So

$$
\left|D_{s}^{\prime} \cap \mathbf{v}\right| \leqq L^{-3}\left|L D^{\prime} \cap L \mathbf{v}\right| \leqq c L^{-3} L^{3 \alpha} L^{\beta},
$$

because from (6.6) and (6.17) we conclude that $L D^{\prime} \cap L \mathbf{v}$ contains at most $c L^{\beta}$ boxes of side $2 L^{\alpha}+1$, since $\mathbf{v} \nless D_{\ell}^{\prime} ;(6.19)$ follows from (6.18) and (6.17). For $\mathbf{u} \notin \hat{D}^{\prime}$, we get, instead of (6.17),

$$
|\mathbf{D} \cap L \mathbf{u}| \leqq c L^{2}
$$


because, using (6.15) and (6.5), $\mathbf{D} \cap L \mathbf{u}$ contains, by definition of $\hat{D}$, and by (6.15), $c L^{\beta}$ unit boxes, plus, possibly, all those on the boundary of $L \mathbf{u}$, i.e. $c L^{2}$. Then, we use (6.18).

d) Equations (6.20), (6.21) follow easily from (6.18) [for (6.20), we use the fact that $D(x)$ is a union of cubes of volume $\left.L^{-3}\right]$, if we show that $D(x)$ cannot intersect two non-adjacent cubes. (u, v are adjacent if $|u-v|<2$ ). Let us prove this by induction. If $D^{\prime}(x) \cap \mathbf{u} \neq \phi, D^{\prime}(x) \cap \mathbf{v} \neq \phi$ with $|u-v| \geqq 2$ then, since $D^{\prime}(x)$ is connected, there must be a $w$, with $|u-w|<2$ and $\left|L D^{\prime}(x) \cap L \mathbf{w}\right| \geqq L$, or $\left|D^{\prime}(x) \cap \mathbf{w}\right| \geqq L^{-2}$ which contradicts (6.18) since $D^{\prime}(x) \subset D_{s}^{\prime}$.

e) We already showed in the proof of a) that $D_{\ell} \subset D_{+}$, so $D_{-} \subset D_{s}$ and, since $D_{+}, D_{-}$ are unions of connected components of $D$, the conclusion holds for $x \in D_{-}$. If $x \in D_{+} \cap L D_{-}^{\prime}$ then we must show $D(x) \cap D_{\ell}=\phi$. But, if $\mathbf{u} \cap D_{\ell} \neq \phi$, then, from (6.8), $n_{v}=1$ for $v=\left[L^{-1} u\right]$ and $\mathbf{v} \subset D_{+}^{\prime}$ by $(6.10,6.11)$. So, if $D(x) \cap L \mathbf{v} \neq \phi$ for $\mathbf{v} \subset D_{+}^{\prime}, D(x)$ $\subset L D^{\prime}(v)$, by construction of $D^{\prime}\left(x \in D_{+}\right.$, so $\left.D(x) \subset D_{+}\right)$. But then, $D(x) \subset L D_{+}^{\prime}$ which contradicts $x \in L D_{-}^{\prime}$.

f) This follows from (6.15) and the definition of ${\widetilde{D^{\prime}}}^{\prime}$ : if $x \in L \mathbf{v}, \mathbf{v} \nless{\widetilde{D^{\prime}}}^{\prime}$, then $|N \cap L \mathbf{u}| \leqq L^{\beta}$ for all $|u-v|<2$. So $|(x+B(L)) \cap N| \leqq c L^{\beta}$. Then, use (6.15).

Proof of Lemma 3. a) Using (6.48), (6.52) and the bound $d\left(\left[L^{-1} X\right]\right) \leqq L^{-1} d(X)+c$, we have

$$
\begin{aligned}
d\left(x^{\prime} \cup y^{\prime} \cup Y^{\prime}\right) \leqq & c k+L^{-1}\left(\sum_{1}^{k}\left|x_{i-1}-y_{i}\right|+\sum_{a_{i} \leqq 4} d\left(x_{i} \cup y_{i} \cup Z_{i}\right)\right. \\
& \left.+\sum_{a_{i}>4} d\left(x_{i} \cup y_{i} \cup Z_{i} \cup W_{i}\right)\right) .
\end{aligned}
$$

We need to analyze the $a_{i}=2,4,5,6$ terms.

For $a_{i}=2$,

$$
d\left(x_{i} \cup y_{i} \cup Z_{i}\right) \leqq d\left(x_{i} \cup y_{i} \cup Z_{i} ; Z_{i} \cap D_{\ell}\right)+\sum_{j} d\left(Z_{i} \cap D_{j}\right),
$$

where $D_{j}$ are the connected components of $D_{\ell}$. By (6.53),

$$
\sum_{j} d\left(Z_{i} \cap D_{j}\right) \leqq C L^{\beta} d\left(\left[L^{-1} Z_{i}\right]\right) \leqq C L^{\beta}\left(L^{-1} d\left(x_{i} \cup y_{i} \cup Z_{i}\right)+c\right)
$$

which yields

$$
d\left(x_{i} \cup y_{i} \cup Z_{i}\right) \leqq 2 d_{2}\left(x_{i}, y_{i}, z_{i}\right)+c L^{\beta} .
$$

Inserting (4) to (1), the $c L^{\beta} L^{-1}$ contributes to the $c k$.

For $a_{i}=4$, we have

$$
d(x \cup y \cup D(x)) \leqq d(y, D(x))+1=d_{4}(x, y, D(x))+1 .
$$

In (5) we used diam $D(x)<1$ which follows from Lemma $1 \mathrm{~d}$ if $D(x) \subset D_{s}$ and this holds by Lemma 1e, since, for $x \notin \bar{D}$, as in (6.48), $\ell(x, y) \neq 0$ only if $x \in D_{+} \cap L D_{-}^{\prime}$.

For $a_{i}>4$, we have

$$
d(x \cup y \cup Z \cup W) \leqq \sum_{i}\left[d\left(x \cup y \cup Y_{i},\left(Y_{i} \cap D_{\ell}\right) \cup W(y)\right)+\sum_{j} d\left(Z \cap D_{j}\right)+d(W(y))\right] .
$$

Now $\sum_{j} d\left(Z \cap D_{j}\right)$ is controlled as above using (6.53) for $Z \neq \phi$, i.e. for $a=5$. Next,

$$
d(W(y)) \leqq c L^{3 \alpha+\beta}
$$


since $b=1$ [see (6.34), (6.36)]. Indeed, $W(y)$ cannot extend over two non-adjacent $L$-cubes, since otherwise we would have an $L$-cube $L \mathbf{w}^{\prime}$ with $\mathbf{w}^{\prime} \in \hat{D}^{\prime}$ and $\left|D \cap L \mathbf{w}^{\prime}\right|$ $\geqq c L L^{-\alpha} \gg L^{\beta}$, as in the proof of Lemma 1d. Thus (7) holds. Since $c L^{3 \alpha+\beta}<L^{1-\alpha}$, we get for $a>4,(6) \leqq 2 d_{a}(x, y, z)+L^{1-\alpha}$ and a) follows.

b) We have

$$
\begin{aligned}
& d\left(x^{\prime} \cup y^{\prime} \cup Y^{\prime} ; Y^{\prime} \cap D_{\ell}^{\prime}\right) \\
& \quad \leqq c k+L^{-1}\left[\sum_{1}^{k}\left|x_{i-1}-y_{i}\right|+\sum d\left(x_{i} \cup y_{i} \cup Y_{i} ; Y_{i} \cap\left(L D_{\ell}^{\prime} \cap D\right)\right)\right],
\end{aligned}
$$

where $Y_{i}$ are $Z_{i}$ or $W_{i}$, see (6.52). To get (8), we used the fact that there is a corridor of width $2 L^{1+\gamma}$ between $\left(L D_{\ell}^{\prime}\right)^{c}$ and $L D_{\ell}^{\prime} \cap \tilde{D}$. This implies that

$$
d\left(X^{\prime} ; X^{\prime} \cap D_{\ell}^{\prime}\right) \leqq c+L^{-1} d\left(X ; X \cap\left(L D_{\ell}^{\prime} \cap \tilde{D}\right)\right) \leqq c+L^{-1} d\left(X ; X \cap\left(L D_{\ell}^{\prime} \cap D\right)\right)
$$

if $\left[L^{-1} X\right]=X^{\prime}$. Now, bound in (8), the second sum by

$$
\begin{aligned}
\sum_{a_{i}=1,3} d\left(x_{i} \cup y_{i} \cup Z_{i}\right)+\sum_{a_{i}=2} d\left(x_{i} \cup y_{i} \cup Z_{i} ; Z_{i} \cap D_{\ell}\right) \\
\quad+\sum_{a_{i}=4} d\left(y_{i}, D(x)\right)+\sum_{a_{i}>4} \sum_{j} d\left(x_{i} \cup y_{i} \cup Y_{j} ;\left(Y_{j} \cap D_{\ell}\right) \cup W\left(x_{i}\right)\right) \\
\quad \times \sum_{i j} d\left(Z_{i} \cap D_{j} \cap L D_{s}^{\prime}\right) \sum_{i}\left(d\left(D\left(x_{i}\right) \cap L D_{s}^{\prime}\right)+d\left(W\left(x_{i}\right) \cap L D_{s}^{\prime}\right)\right) .
\end{aligned}
$$

Now proceed as in a). Since $D_{s}^{\prime} \cap \widetilde{D}^{\prime}=\phi$, we get

$$
\sum_{i j} d\left(Z_{i} \cap D_{j} \cap L D_{s}^{\prime}\right) \leqq c L^{\beta}\left(L^{-1} \sum_{j} d\left(Y_{j}^{\prime} \cap D_{s}^{\prime}\right)+c\right)
$$

where $Y_{j}^{\prime}$ are the c.c. of $Y^{\prime}$. Similarly the right-hand side of $(10)$ bounds the two last sums of (9). Since

$$
\sum_{j} d\left(Y_{j}^{\prime} \cap D_{s}^{\prime}\right) \leqq d\left(x^{\prime} \cup y^{\prime} \cup Y^{\prime} ; Y^{\prime} \cap D_{\ell}^{\prime}\right)
$$

we get the claim of $b$ )

c) We have

$$
d\left(y^{\prime}, D^{\prime}\left(x^{\prime}\right)\right) \leqq L^{-1}|P|
$$

for any path $P$ joining $L D^{\prime}\left(x^{\prime}\right)$ to $L y^{\prime}$, and not intersecting $L D^{\prime}\left(x^{\prime}\right)$, except at one point. Now, because of the corridors around $L \widetilde{D}^{\prime}$ in $(6.5)$, and because $D^{\prime}\left(x^{\prime}\right)$ is connected,

$$
L \tilde{D}^{\prime} \cap\left(L\left(D^{\prime}\left(x^{\prime}\right)+B\left(2 L^{\gamma}\right)\right) \backslash L D^{\prime}\left(x^{\prime}\right)\right)=\phi
$$

So

$$
\left|P \cap\left(D+B\left(L^{\alpha}\right)\right) \cap L\left(D^{\prime}\left(x^{\prime}\right)+B\left(2 L^{\gamma}\right)\right)\right| \leqq c L^{3 \alpha+\beta}\left(L^{-1}|P|+c\right),
$$

and, using $|P|=|P \backslash E|+|P \cap E|$

$$
|P| \leqq 2\left|P \backslash\left(\left(D+B\left(L^{\alpha}\right)\right) \cap L\left(D^{\prime}\left(x^{\prime}\right)+B\left(2 L^{\gamma}\right)\right)\right)\right|+c L^{3 \alpha+\beta} .
$$

Inserting (13) in (11) and using the definition of $d_{a}$ we have (6.67) because $W\left(L x^{\prime}\right)$ $C L D^{\prime}\left(x^{\prime}\right)[\operatorname{see}(6.34)]$ and all the other terms after the semicolon in $d_{a}, a=2,5,6$ and $D\left(x_{i}\right)$, for $a=4$, are contained in $\left(D+B\left(L^{\alpha}\right)\right) \cap L\left(D^{\prime}\left(x^{\prime}\right)+B\left(2 L^{\beta}\right)\right)$. 
Proof of Lemma 4. Consider $a=6$ first. It suffices to bound

$$
\sum_{t=1}^{L^{2}} \int_{W^{c}} d x T_{W \cap \bar{D}^{c}}^{t}(y, x) e^{d(x, W)} \leqq c
$$

with $y \in W$. We get an upper bound by replacing $T_{W \cap \bar{D}}^{t} c$ by $T_{W}^{t}$. Define $\pi: \mathbf{Z}^{3} \times \mathbf{Z}^{3} \rightarrow \mathbf{R}$ by $\pi(x, y)=T(x-y)$ if $x, y \in W, \pi(x, y)=\delta_{x y}$ if $x, y \notin W, \pi(x, y)$ $=T(x, y) e^{d(y, W)}$ if $y \notin W, x \in W$ and $\pi(x, y)=0$ otherwise. Then the left-hand side of (14) is bounded by

$$
\int d x \pi^{L^{2}}(x, y)
$$

Since $\int_{W} \pi(x, y) d y \leqq 1$ if $x \in W, p(x, y)=0$ if $x \notin W, y \in W$ and $\int \pi(x, y) d y=1$ if $x \notin W$, we get

$$
(15) \leqq \sup _{x \in W} \int_{W^{c}} d y T(x-y) e^{|x-y|} \leqq c,
$$

$a=5$ remains to be proven. Recall (6.46) and (6.47) and consider first the $k=1$ term. This is similar to the $a=6$ case above, the only change being that $T$ is replaced in (14) by $T+\hat{b}$. We claim that, if $d\left(x \cup y \cup Y\right.$; W) $>L^{\gamma}$ [see (6.43)], then

$$
d\left(x \cup y \cup Y ;\left(Y \cap D_{\ell}\right) \cup W\right)>L^{\gamma} .
$$

Assuming this, we get, for $a=1,2,3$,

$$
\sum_{a, Y} \int d x\left|Q_{a Y}(y, x)\right| \chi(Y) e^{\frac{1}{2} d\left(x \cup y \cup Y ;\left(Y \cap D_{\ell}\right) \cup W\right)} \leqq e^{-L^{\nu / 2}} .
$$

Indeed, using (17),

$$
\chi(Y) \leqq \exp \left(-\frac{L^{\gamma}}{2}+\frac{1}{2} d\left(x \cup y \cup Y ;\left(Y \cap D_{\ell}\right) \cup W\right)\right)
$$

and $d\left(x \cup y \cup Y ;\left(Y \cap D_{\ell}\right) \cup W\right) \leqq d_{a}(x, y, Y)$ for $a=1,2,3$ (18) follows from the inductive bounds $(6.25,6.26)$ and $(5.11)$.

$(18)$ and $\int(T+b)(x, y) d y=1$ implies

$$
\int|(T+\hat{b})(x, y)| d y \leqq 1+c e^{-L^{\nu} / 2}
$$

Also we have, for $x \in W$,

$$
\int_{W c}|(T+\hat{b})(x, y)| e^{d(y, W)} d y \leqq c
$$

because we may use (16) for the $T$ part and (6.25-6.27) for the $\hat{b}$ part. Then, for $k=1$, we argue as we did above for $a=5$. The bound we just proved replaces (15), while (19) replaces $\int \pi(x, y) d y \leqq 1$; in the modified bound on (15), we get a factor $\left(1+c e^{-L^{\nu} / 2}\right)^{L^{2}}<c$.

For $k>1$, we use (18): this controls the $L^{2}$ coming from the sum over $t_{i}$ and the $y$ integrals are bounded using (19).

To prove (17) we observe that, by definition of $W,(6.34), W$ is at a distance at least $L^{\alpha}$ from $D_{+} \backslash W$ because $W$ is a connected component of $\left(\left(D_{+}+B\left(L^{\alpha}\right)\right) \cup D\right)$ or of $L D^{\prime}$. But $D_{\ell} \subset D_{+}$, hence $W$ is at a distance at least $L^{\alpha}$ from $D_{\ell} \backslash W$. So, (17) holds.

Proof of Lemma 5. We shall use the following Green's function bounds on $T(x-y)$, all of which follow from the Fourier transform bounds of Proposition 5.1. 
Let $E$ be a set of $L^{-1}$ cubes and let $t \leqq L^{2}$. Then,

and

$$
\begin{gathered}
\int_{E} d y T^{t}(x-y) e^{2 L^{-1}|x-y|} \leqq c|E|(t+1)^{-3 / 2} \exp \left(-\frac{d(x, E)}{t^{1 / 2}}\right) \\
\int d y T^{t}(x-y) e^{2 L^{-1}|x-y|} e^{-d(y, E) / 4} \geqq c|\mathbf{E}|(t+1)^{-3 / 2} \exp \left(-\frac{d(x, E)}{t^{1 / 2}}\right) .
\end{gathered}
$$

Note that we get $|\mathbf{E}|$ here instead of $|E|$ and $\mathbf{E}$ is, by definition, a set of unit cubes.

Summing over $t$, we get

$$
\sum_{t=0}^{L^{2}}(20) \leqq c \min \left(L^{2}, \frac{|E|}{d(x, E)+1}\right) e^{-d(x, E) / L},
$$

and similarly for the sum over $t$ of $(21)$, with $|E|$ replaced by $|\mathbf{E}|$.

The proof of the lemma reduces to a combination of Lemma 4 and the Green's function estimates above.

a) Equation (6.74) follows immediately from (6.25). For (6.75), we use

$$
1 \leqq c \sum_{u \in \mathbf{Z}^{d}} e^{-d(y, \mathbf{N} \cap L \mathbf{u}) / 2} e^{d(y \cup z \cup Z) / 2}
$$

which holds for $N(Z) \neq-\infty$, by Lemma 1a. Since $Q_{1 Z}=0$ if $N(Z)=-\infty$ (by Proposition 6.1 and Lemma 6.1), we may insert (23) in (6.71), and get

$$
\begin{aligned}
\tilde{A}_{1}(x, t) \leqq & c \sum_{Z} \sum_{u \in \mathbf{Z}^{d}} \int d y d z \chi(y \notin \bar{D}) T^{t}(x-y) e^{2 L^{-1}|x-y|} e^{-d(y, \mathbf{N} \cap L \mathbf{u}) / 2} \\
& \times\left|Q_{1 Z}(y, z)\right| e^{d(x \cup y \cup Z)} L^{-N(Z) / 2} .
\end{aligned}
$$

Using (6.25), the $z$ integral and the $Z$ sum are bounded by $L^{-\alpha / 2}$. Use (21) with $E=\mathbf{N} \cap L \mathbf{u}$, to get

$$
(24) \leqq c(t+1)^{-3 / 2} L^{-\alpha / 2}\left(\sum_{u \in D^{\prime}}|\mathbf{N} \cap L \mathbf{u}| e^{-\frac{x}{L}-u}+\sum_{u \in D^{\prime}}|\mathbf{N} \cap L \mathbf{u}| e^{-\frac{L}{4}} e^{-\frac{x}{L}-u}\right),
$$

where we use the fact that $d\left(y \notin, \mathcal{L}^{\prime} \mathbf{u}\right) \geqq L$ if $u \in \hat{D}^{\prime}$ and $y \notin \bar{D}$. Now, using $|\mathbf{N} \cap L \mathbf{u}| \leqq L^{\beta}$, by definition, for $u \notin \hat{D}^{\prime}$, and $|\mathbf{N} \cap L \mathbf{u}| \leqq L^{3}$ for $u \in \hat{D}^{\prime}$, we get (6.75).

For (6.76), we proceed in a similar manner. Write in (6.71)

$$
\sum_{Z}=\sum_{Z \cap D_{+} \neq \phi}+\sum_{Z \cap D_{+}=\phi}
$$

For the sum over $Z \cap D_{+} \neq \phi$, use the analogue of (23) with $d\left(y, D_{+} \cap L \mathbf{u}\right)$ instead of $d(y, \mathbf{N} \cap L \mathbf{u})$ and use (22) instead of (21): this gives the first term in (6.76). For the sum over $Z \cap D_{+}=\phi$ we use $N(Z)<0$ and the fact that $Q_{1 Z}=0$ if $N(Z)=-\infty$. If $N(Z)<0, N(Z) \leqq 1+2 \alpha$ since $N_{\mathbf{u}} \in(1-2 \alpha) Z$. So, in that sum we may insert

$$
1 \leqq L^{-\frac{1}{2}+\alpha} L^{-\frac{N(Z)}{2}} \text {. }
$$

Now, reasoning as above we get a bound $L^{-\frac{1}{2}+\alpha} c L^{\beta}\left(d\left(x, D_{-}\right)+1\right)^{-1}$ which is smaller than $L^{-\alpha}$.

b) is obvious, given Lemma 4 and $L^{2} \ll e^{L^{\gamma / 2}}$.

c) If $x \in D_{+} \backslash \bar{D},(6.78)$ follows from Lemma 4. If $x \notin D_{+} \backslash \bar{D}$, we have $x=y$ in (6.72) because $t=0\left(T^{0}(x-y)=\delta(x-y)\right)$. In (6.72), $y \notin \bar{D}$, so $x=y \notin D_{+}$. But

$$
Q_{4 Z}(y, z)=Q_{4 Z}(x, z)=\ell(x, z)=0 \quad \text { for } \quad x \notin D_{+} .
$$


For $r \neq 0$, we bound the $z$ integral and the $Z$ sum in (6.72) by a constant, using Lemma 4 . The $y$ integral in (6.72) is controlled by the fact that $y \notin \bar{D}$ but $y \in D_{+}$, otherwise $Q_{4 z}(y, z)=0$. We bound it by

$$
\sum_{u \in \mathbf{Z}^{d}} \int d y \chi\left(y \in\left(D_{+} \backslash \bar{D}\right) \cap L \mathbf{u}\right) T^{t}(x-y) e^{2 L^{-1}|x-y|} \leqq t^{-3 / 2} \sum_{u \notin D^{\prime}} \mid D_{s} \cap L \mathbf{u}^{-\left|\frac{x}{L}-u\right|}
$$

using (20) with $E=\left(D_{+} \backslash \bar{D}\right) \cap L \mathbf{u}$, and using $D_{+} \backslash \bar{D}=D_{+} \# L D_{-}^{\prime} \subset D_{s}$, which follows from (6.31) and Lemma 1e. Moreover, we used $\left(D_{+} \backslash \bar{D}\right) \cap L \mathbf{u}=\phi$ if $u \in \widetilde{D}^{\prime}$.

By Lemma 1c,

$$
(28) \leqq t^{-3 / 2} c L^{-2},
$$

which proves (6.79). $(6.80)$ is obtained from $(6.78,6.79)$ by summing over $t$. d) Since $\bar{D} \backslash L \widetilde{D}^{\prime}=L D_{+}^{\prime} \cap D_{+} C D$, we may write in (6.73),

$$
\chi\left(\mathrm{y} \in \overline{\mathrm{D}} \backslash L \tilde{D}^{\prime}\right)=\sum_{u \notin D^{\prime}} \chi\left(y \in D_{\ell} \cap L \mathbf{u}\right)+\chi\left(y \in D_{s} \cap L \mathbf{u}\right) .
$$

The $z$ integral and the $Z, t^{\prime}$ sums in (6.73) are bounded by a constant, using Lemma 4. Lemma $1 b$ and $c$ give

$$
\left|D_{\ell} \cap L \mathbf{u}\right| \leqq c L^{\beta}, \quad\left|D_{s} \cap L \mathbf{u}\right| \leqq L^{-2}
$$

(for $u \notin \widetilde{D^{\prime}}$ ).

Then, using (22) with $E=D_{\ell} \cap L \mathbf{u}$ or $E=D_{s} \cap L \mathbf{u}$ for the $y$ integral in (6.73), we get the two terms in (6.81).

Proof of Lemma 6. a) For $a^{\prime}=5,6$ (6.84) follows from (6.85) (6.86) to be proven below. If $a$ or $a^{\prime}=2,3$ (6.84) follows trivially from Lemma 4. So we have only to consider $a=1,4$, and $a^{\prime}=1,4$ or $a=5,6$ and $a^{\prime}=1$.

Consider first $a, a^{\prime} \in\{1,4\}$. If $a=4, a^{\prime}=1$ we may use (6.76) to bound $A_{\alpha}(z)$ by $2 L^{-\alpha / 3}$. The $z$ integral and the $Z$ sum may be bounded by

$$
c \chi\left(y \in D_{+}\right)
$$

using Lemma 4 and $a=4$.

The sum over $t$ and the $y$ integral in (6.82) are bounded using (28) [plus 0(1) for $t=0]$. Combining these, (6.84) holds in that case. If $a=1, a^{\prime}=4$, we get a constant as a bound on $A_{a^{\prime}}(z)$ from (6.80) and the rest is bounded by $2 L^{-\alpha / 3}$ from (6.76); $a=1$, $a^{\prime}=1$ is treated similarly.

Finally, consider $a=4, a^{\prime}=4$. Insert in (6.82),

$$
1=\chi\left(z \in D_{+} \backslash \bar{D}\right)+\chi\left(z \notin D_{+} \backslash \bar{D}\right) .
$$

For $z \notin D_{+} \backslash \bar{D}$ we get a bound $c L^{-\alpha}$ on $A_{a^{\prime}}(z)$ from (6.80) and we are done. For $z \in D_{+} \backslash \bar{D}$, we bound $A_{a^{\prime}}(z)$ by a constant and we notice that $D_{+} \backslash \bar{D}=D_{+} \cap L D_{-}^{\prime}$ $C D_{s} \backslash L \hat{D}^{\prime}$ because of Lemma 1e and $\hat{D}^{\prime} \subset D_{\ell}^{\prime} \subset D_{+}^{\prime}$. We write

$$
\begin{aligned}
& \int d z\left|Q_{4 z}(y, z)\right| e^{L^{-} \gamma_{d(z, D(y))}} \chi\left(z \in D_{+} \backslash \bar{D}\right) \\
& \quad \leqq e \int d z\left|Q_{4 Z}(y, z)\right| \chi\left(z \in D_{s} \backslash L \hat{D}^{\prime}, d(z, D(y)) \leqq L^{y}\right)+\exp \left(-c L^{y}\right),
\end{aligned}
$$

where we used (6.27) for the integral over $d(z, D(y))>L^{2 \gamma}$. We claim that

$$
(30) \leqq c L^{-\alpha / 3}
$$

for $y \notin \bar{D}, y \in D_{+}$. 
To show this, we use (6.28): if $y \notin \bar{D}$ but $y \in D_{+}$, it means that $N_{u^{\prime}}^{\prime}<0$ for $u^{\prime}$ such that $L \mathbf{u}^{\prime} \in y$. So, from (6.10) we see that

$$
N_{v} \leqq N_{u}^{\prime}+2-2 \alpha \leqq-1+2 \alpha+2-2 \alpha \leqq 1 \text { for } y \in \mathbf{v} \subset L \mathbf{u}^{\prime} .
$$

This implies $\beta_{v} \geqq 1-L^{-1}$ and, using (6.28), proves (31). We use (28) [plus 0(1) for $t=0]$ to control the $t$ sum and the $y$ integral. This gives (6.84) for $a=4, a^{\prime}=4$.

Now let $a=5,6, a^{\prime}=1$. Here we combine (6.76) for $A_{a^{\prime}}(z)$ and (6.81) for the rest which is just $A_{a}(x)$ for $a=5,6$. This yields (6.84).

b) For (6.85), we use (6.81) on $A_{a^{\prime}}(z)$. We get

$$
\begin{gathered}
B_{1 a^{\prime}}(x) \leqq \sum_{Z, t} \int d y d z \chi(y \notin \bar{D}) T^{t}(x-y) \quad \text { principle } \\
\times e^{2 L^{-1}|x-y|}\left|Q_{1 Z}(y, z)\right| e^{L^{-\gamma} d(y \cup z \cup Z)}\left(c L^{\beta}\left(d\left(z, D_{\ell}\right)+1\right)^{-1}+L^{-\alpha}\right) .
\end{gathered}
$$

Divide the $z$ integral into $|z-y| \leqq L^{\gamma}$ and $|z-y|>L^{\gamma}$. The second part contributes to $L^{-\alpha}$ in (6.85). For the first, we have trivially

$$
\left(d\left(z, D_{\ell}\right)+1\right)^{-1} \leqq L^{y}\left(d\left(y, D_{\ell}\right)+1\right)^{-1} .
$$

Now insert (23) and bound the resulting $z$ integral and $Z$ sum, using Lemma 4, by $L^{-\alpha / 2}$. So we have only to bound

$$
c L^{-\frac{\alpha}{2}+\beta+\gamma} \sum_{t, u \in \mathbf{Z}^{d}} \int d y T^{t}(x-y) e^{2 L^{-1}|x-y|} e^{-d(y, \mathbf{N} \cap L \mathbf{u}) / 2} \chi(y \notin \bar{D})\left(d\left(y, D_{\ell}\right)+1\right)^{-1}
$$

by $\left.L^{-\frac{\alpha}{3}}\left(d\left(x, D_{\ell}\right)+1\right)\right)^{-1}$ which follows, arguing as in (25), from

$$
d\left(x, D_{\ell}\right) \leqq(d(x, \mathbf{N} \cap L \mathbf{u})+1) e^{d(y, \mathbf{N} \cap L \mathbf{u}) / 4}\left(d\left(y, D_{\ell}\right)+1\right)
$$

and (22) with $\mathbf{E}=\mathbf{N} \cap L \mathbf{u}$.

For (6.86), we consider $a=4, a^{\prime}=5,6$. Divide the $z$ integral into $d(z, D(y)) \leqq L^{\gamma}$ and $d(z, D(y))>L^{\gamma}$. For the latter, we may use Lemma 4. Since $y \in D_{+} \backslash \bar{D}, D(y) \subset D_{s}$, and $\operatorname{diam}(D(y))<1$ by Lemma $1 \mathrm{e}$, d. So, $y \in D_{+} \backslash \bar{D}$ and $d(z, D(y)) \leqq L^{\gamma}$ imply $d\left(z, D_{\ell}\right)$ $\geqq L^{\alpha}-L^{\gamma}$, since $D_{\ell} \subset L D_{+}^{\prime} \cap D_{+}, y \notin L D_{+}^{\prime}$ (since $\left.y \in D_{+} \backslash \bar{D}\right)$ and $L D_{+}^{\prime}$ has, by construction, a corridor of width $L^{\alpha}$ around $D_{+} \cap L D_{+}^{\prime}$. Now, using $d\left(z, D_{\ell}\right) \geqq \frac{L^{\alpha}}{2}$, the bound (6.81) on $A_{a^{\prime}}(z)$ and (6.80) for the rest, we get (6.86).

c) Divide the $z$ integral in (6.83) into $d(z, W(y)) \leqq L^{\gamma}$ and $d(z, W(y))>L^{\gamma}$. By construction, $d\left(\partial W(y), D_{+}\right) \geqq L^{\alpha}$, see (6.34). Since $z \notin W(y), d(z, W(y)) \leqq L^{\gamma}$ implies $d\left(z, D_{+}\right) \geqq L^{\alpha}-L^{\gamma} \geqq \frac{L^{\alpha}}{2}$. Since $D_{\ell} \subset D_{+}$, we may use (6.81) on $A_{a^{\prime}}(z)$ and then again (6.81) for the rest. If $d(z, W(y))>L^{\gamma}$, we use the exponential decay coming from Lemma 4.

Proof of Lemma 7. a) Notice that $J$ collects indices where $b_{j}=2$, i.e. $W\left(y_{j}\right)$ $=$ connected component of $L D^{\prime}$ and $y_{j} \in L \widetilde{D}$ or $j=0$. We shall use the fact that, in order to connect $W\left(y_{j^{\prime}}\right) \cap L D^{\prime}$ to $W\left(y_{j}\right) \cap L \widetilde{D}$ for $j^{\prime} \neq j$ the path has to cross a corridor of size $2 L^{1+\gamma}$ [see (6.5)], where, by definition $D$ is sparse (the corridors are not in $\widetilde{D}$ ). This is true also for $j=0$, since $x^{\prime} \in D^{\prime}$ and even if $W\left(y_{j^{\prime}}\right)=W\left(y_{j}\right)$, i.e. if the walk revisits the same set, because of the condition $x_{j} \in W\left(y_{j}\right)$ in (6.49): the walk has to leave $W\left(y_{j^{\prime}}\right)$ before revisiting $W\left(y_{j^{\prime}}\right) \cap L \widetilde{D}$.

So, let $G$ be a connected graph joining $L \tilde{D}^{\prime} \cap W\left(y_{j}\right)$ to $W\left(y_{j}\right)^{c}$. The (possibly disconnected) graph $G \backslash\left(D+B\left(L^{\alpha}\right)\right)$ becomes connected when connected components of $D+B\left(L^{\alpha}\right)$ are contracted to a point. 
First, observe that the minimum of $\left|G \backslash\left(D+B\left(L^{\alpha}\right)\right)\right|$ overt all such $G$ is less than the left-hand side of (6.102). This is obvious, given what we said above, because, for all $d_{a}$ 's in (6.103), the sets after the semicolon that are inside $W\left(y_{j}\right)$ are also inside $D+B\left(L^{\alpha}\right)$ : by definition for all $i$, with $j^{\prime}<i<j$, we have $b_{i}=1$ and therefore $W_{i} \subset D$ $+B\left(L^{\alpha}\right)$. Next, we claim that

$$
\left|G \backslash\left(D+B\left(L^{\alpha}\right)\right)\right| \geqq \frac{3}{2} L^{1+\gamma}
$$

which, then, yields (6.102). Certainly $|G| \geqq 2 L^{1+\gamma}$ because of the corridors in (6.5). Write

$$
|G|=\left|G \backslash\left(D+B\left(L^{\alpha}\right)\right)\right|+\left|G \cap\left(D+B\left(L^{\alpha}\right)\right)\right| .
$$

But $G$ is in $W\left(y_{j}\right) \backslash L \tilde{D}^{\prime}$, hence

$$
\left|G \cap\left(D+B\left(L^{\alpha}\right)\right)\right| \leqq c L^{\beta} L^{3 \alpha}\left|\left[\frac{G}{L}\right]\right|,
$$

since, for each $u$ in $\left[\frac{G}{L}\right],|D \cap L \mathbf{u}| \leqq c L^{\beta}$ by Lemma $1 \mathrm{~b}$. Now,

$$
\left|\left[\frac{G}{L}\right]\right| \leqq L^{-1}|G|+c .
$$

This and (34), (35) proves (33).

b) This uses the corridor of size $L$ in $L \widetilde{D}^{\prime}$, around $L \widetilde{D}^{\prime}$. Consider a connected graph $G$ joining $x, y$ and $Z$. Again, the minimum of $\left|G \backslash\left(D+B\left(L^{\alpha}\right)\right)\right|$ for such graphs is less than $d_{a}(y, x, Z)$ for $a=2,5$. But since $Z \cap L \widehat{D}^{\prime} \neq \phi, y \notin L \widetilde{D}^{\prime}$ and $G$ is connected, there must be a $\mathbf{u} \notin \hat{D}^{\prime}$ with $|G \cap L \mathbf{u}| \geqq L$. Since $\mathbf{u} \notin \hat{D}^{\prime}$,

$$
\left|\left(G \backslash\left(D+B\left(L^{\alpha}\right)\right)\right) \cap L \mathbf{u}\right| \geqq L-c L^{\beta+3 \alpha},
$$

because $\mathbf{D}$ contains $c L^{\beta}$ boxes in $L \mathbf{u}$, except possibly on the boundary of $L \mathbf{u}$. This concludes the proof of $b$ ).

Proof of Lemma 8. This is similar to the proof of Lemma 7, using the corridor $B\left(2 L^{\gamma}\right)$ in (6.60). Let $G$ be a connected graph joining $L D^{\prime}\left(x^{\prime}\right)$ to $\left(L D^{\prime}\left(x^{\prime}\right)+2 B\left(L^{1+\gamma}\right)\right)$. The minimum of $\left|G \backslash\left(D+B\left(L^{\alpha}\right)\right)\right|$ is larger than the left-hand side of $(6.106)$, and, obviously, $|G| \geqq 2 L^{1+\gamma}$. But in the corridor $\left(L D^{\prime}\left(x^{\prime}\right)+2 B\left(L^{1+\gamma}\right)\right) \backslash L D^{\prime}\left(x^{\prime}\right)$ there cannot be any $L \mathbf{u}$, with $u \in \tilde{D}^{\prime}$ because $D^{\prime}\left(x^{\prime}\right)$ is connected, see (6.5). So $\left|G \cap\left(D+B\left(L^{\alpha}\right)\right)\right| \leqq c L^{\beta+3 \alpha}\left|\left[\frac{G}{L}\right]\right|$ and using (34), (36), we get (6.106).

Acknowledgements. J. B. thanks Rutgers University and the Institute for Advanced Study for hospitality during the work. We thank S. Goldstein for useful discussions. The work was supported in part by NSF grant DMS-8903041.

\section{References}

1. Sinai, Y.G.: Limiting behavior of a one-dimensional random walk in a random medium. Theory Prob. Appl. 27, 256 (1982)

2. Marinari, E., Parisi, G., Ruelle, D., Windey, P.: Random walk in a random environment and $1 / f$ noise. Phys. Rev. Lett. 50, 1223 (1983); on the interpretation of $1 / f$ noise. Commun. Math.

Phys. 89, 1 (1983) 
3. Fisher, D.: Random walks in random environments. Phys. Rev. A 30, 960 (1984)

4. Derrida, B. Luck, J.M.: Diffusion on a random lattice: weak-disorder expansion in arbitrary dimension. Phys. Rev. B 28, 7183 (1983)

5. Luck, J.M.: Diffusion in a random medium: a renormalization group approach. Nucl. Phys. B 225, 169 (1983)

6. Durrett, R.: Multidimensional random walks in random environments with subclassical limiting behavior. Commun. Math. Phys. 104, 87 (1986)

7. Bouchaud, J.P., Comtet, A., Georges, A., Le Doussal, P.: Anomalous diffusion in random media of any dimensionality. J. Physique 48, 1445 (1987)

8. Bramson, M., Durrett, R.: Random walk in random environment: a counterexample? Commun. Math. Phys. 119, 119 (1988)

Bramson, M.: Random walk in random environment: A counterexample without potential. Preprint

9. Papanicolaou, G., Varadhan, S.R.S.: Boundary value problems with rapidly oscillating random coefficients in "Random Fields," Fritz, J., Lebowitz, J., Szasz, D. (eds.). Janos Bolyai Series, p. 835. Amsterdam: North-Holland 1981

10. Papanicolaou, G., Varadhan, S.R.S.: Diffusion with random coefficients. In: Statistics and probability: essays in honor of C.R. Rao. Kallianpur, G., Krishaniah, P.R., Gosh, J.K. (eds.), p. 547. Amsterdam: North-Holland 1982

11. Anshelevich, V.V., Khanin, K.M., Sinai, Ya.G.: Symmetric random walks in random environments. Commun. Math. Phys. 85, 449 (1982)

12. Kunnemann, R.: The diffusion limit of reversible jump processes in $\mathbf{Z}^{d}$ with ergodic random bond conductivities. Commun. Math. Phys. 90, 27 (1983)

13. Lawler, G.: Weak convergence of a random walk in a random environment. Commun. Math. Phys. 87, 81 (1982)

14. De Masi, A., Ferrari, P.A., Goldstein, S., Wick, D.: An invariance pricniple for reversible Markov processes. Applications to random motions in random environments. J. Stat. Phys. 55, 787 (1989)

15. Bricmont, J., Kupiainen, A.: Phase transition in the $3 d$ random field Ising model. Commun. Math. Phys. 116, 539 (1988)

16. Billingsley, P.: Convergence of probability measures. New York: Wiley 1968

Communicated by T. Spencer 